\title{
Conjugate Buoyant Convective Transport of Different Nanofluids in An Enclosed Annular Geometry
}

\author{
M. Sankar \\ Presidency University \\ N. Keerthi Reddy \\ Presidency University \\ Younghae Do ( $\nabla$ yhdo@knu.ac.kr) \\ Kyungpook National University
}

\section{Research Article}

Keywords: vertical, Conjugate, nanofluids, cylindrical

Posted Date: January 27th, 2021

DOl: https://doi.org/10.21203/rs.3.rs-147388/v1

License: (c) (i) This work is licensed under a Creative Commons Attribution 4.0 International License.

Read Full License 


\title{
Conjugate buoyant convective transport of different nanofluids in an enclosed annular geometry
}

\author{
M. Sankar ${ }^{1,+}$, N. Keerthi Reddy ${ }^{1,+}$, and Younghae Do ${ }^{2, *}$ \\ ${ }^{1}$ Department of Mathematics, School of Engineering, Presidency University, Bengaluru, 560064, India. \\ ${ }^{2}$ Department of Mathematics, KNU-Center for Nonlinear Dynamics, Kyungpook National University, Daegu, 41566, \\ Republic of Korea. \\ *Email:yhdo@knu.ac.kr \\ +These authors contributed equally to this work.
}

\begin{abstract}
A vertical annular configuration with differently heated cylindrical surfaces and horizontal adiabatic boundaries is extensively studied due to many industrial applications. In this paper, we investigate the effects of conjugate buoyant heat transport within water based nanofluid with different nanoparticles such as alumina, titanium oxide or copper, which is contained in the gap of the enclosed annulus. The enclosed annulus is constituted by a thick inner cylinder with a constant high temperature, an exterior boundary with a constant low temperature and thermally insulated upper and lower surfaces. By investigating heat transport for broad spectrum of Rayleigh number, solid wall thickness, thermal conductivity ratio and nanoparticle volume fraction, we found that the influence of wall thickness on thermal dissipation rate along wall and interface greatly depend on conductivity ratio and vice-versa. In particular, we uncover that the choice of nanoparticle in a nanofluid and its concentration are key factors in enhancing the thermal transport along the interface. Specially, copper based nanofluids produces higher heat transport among other nanoparticles, and increasing nanoparticle concentration leads to enhanced thermal dissipation along interface. Our results are applicable to choose nanofluids along with other critical parameters for the desired heat transport.
\end{abstract}

\section{Introduction}

Buoyant thermal transport of conventional fluids as well as nanofluids in various finite-sized geometries have been widely investigated through theoretical simulations and experimental visualizations. This is mainly due to the direct relevance of these geometries in many vital applications ranging from cooling of electronic components to safety measures of nuclear reactors. In particular, amongst the finite-sized geometries, the annular space formed by two up-right co-axial cylindrical tubes with different heating of side boundaries and insulated horizontal surfaces is considered as a suitable model problem aptly describing the physical configuration of the above stated applications. The pioneering attempt to numerically explore the buoyant convection in an upright annular space was by Davis and Thomas. ${ }^{1}$ They performed simulations for wider spectrum of parameter ranges and proposed heat transport correlations in different flow regimes. Later, Kumar and Kalam ${ }^{2}$ made numerical simulations of convective flow, thermal transport analysis and reported the discrepancies existing in the results of Davis and Thomas, ${ }^{1}$ and suggested new correlations to predict the thermal transport rates. Convective flow of different liquids in an annular enclosure with $\lambda=5.338$ has been experimentally investigated by Prasad and Kulacki ${ }^{3}$ by considering three aspect ratios. Few applications such as crystal growth processes require mechanisms to resist or dampen the convective flow to design defect-free products and this can be achieved by employing magnetic force by considering different fluids. ${ }^{4-7}$ Sankar and co-workers ${ }^{8,9}$ made detailed investigations to analyze the impacts of thermal sources on buoyant convective motion of air in a discretely heated porous and non-porous annulus and observed discrete heating could enhance thermal transport compared to complete heating of wall. Later, Wang et al. ${ }^{10}$ investigated transient buoyant flow 
in the same geometry and reported new correlations to predict the thermal transport rates. The size and positional influence of thermal sources on hydrodynamic stability has been numerically examined by Mebarek-Oudina ${ }^{11}$ in an upright annular domain. Recently, Husain and Siddiqui ${ }^{12}$ made an experimental analysis of unsteady buoyant convective flow of water in tall and narrow annular region and also presented theoretical simulations through a commercial CFD model. It is worth to mention that the above investigations mainly addresses the buoyant flow and associated transport processes in a vertical annular chamber without taking account of wall thickness effect.

The addition of nano-sized particles in conventional liquids could effectively enhance the thermal transport rates and is substantiated through the predictions made by many theoretical simulations and experimental observations. ${ }^{13,14}$ Thermal transport analysis of different nanofluids in finite shaped geometries have also received a great amount of attraction due to the requirement of effective cooling of electronic equipments. One of the earliest attempt to investigate buoyant motion of $\mathrm{Al}_{2} \mathrm{O}_{3}$ nanofluid in an annular geometry is by Abouali and Falahatpisheh. ${ }^{15}$ They performed extensive numerical simulations by considering wide spectrum of parameter ranges and proposed thermal transport correlations for square and annular geometries. Cadena-de la Peña et al. ${ }^{16}$ conducted experiments to analyze cooling mechanisms of oil-based nanoliquids by considering two different nanoparticles and found thermal transport enhancement with nanofluids. The impacts of discrete thermal sources of different lengths and locations on buoyant motion of nanofluids in an annular domain reveals interesting flow features and enhanced thermal transport as compared to uniform or complete heating. ${ }^{17,18}$ Recently, Keerthi and Sankar ${ }^{19}$ presented numerical simulations to reveal the consequences of different non-uniform heating of annular boundaries on the convective motions of $\mathrm{Cu}$-based nanofluid and identified an appropriate heating condition to enhance the thermal dissipation rates. The convective motions of various nanofluids in horizontal and tilted annular configurations with and without fins have also been reported..$^{20,21}$

Among the finite shaped enclosures, rectangular and square geometries have been widely used in analyzing the nanofluid buoyant motion by considering various constraints affecting the flow and thermal transport mechanisms. Khanafer et $a l .{ }^{22}$ presented detailed analysis on thermal transport enrichment for $\mathrm{Cu}$ - based nanofluids in a square geometry and proposed a theoretical model and heat transport correlations to estimate nanofluid thermal performance. Later, this study was extended by Jou and Tzeng ${ }^{23}$ by taking nanoparticle dispersion in to consideration and aspect ratio. Many investigations analyzed the buoyant motion and thermal dissipation of different nanofluids in two-dimensional plane geometries by considering various models for fluid properties and identified an appropriate model for thermal transport enhancement. ${ }^{24-26}$ Through numerical predictions it has been observed that thermal dissipation enrichment in an enclosure predominantly depends on the choice of nanoparticle as well as the thermal conditions. ${ }^{27-29}$ Roy $^{30}$ examined nanofluid buoyant motion in the annular section between a square geometry and three distinct interior geometries, such as circular or elliptical or rectangular cylinder and found the inner shapes has profound impacts on thermal dissipation rates compared to a square geometry. Using combined Lagrangian and Eulerian modeling, Sharaf et al. ${ }^{31}$ investigated the convective motion and nanoparticle dissemination in a microchannel formed by parallel plates and brought out inaccuracies in the existing nanofluid model. The impacts of three different arrangements of conductive baffles on nanofluid motion and associated thermal behavior in a square geometry has been performed by Bendaraa et al. ${ }^{32}$ and noticed that the fin location has vital role in effective controlling of the flow movement and thus thermal dissipation rates. Buoyant nanofluid motion and the associated thermal dissipation rates are highly sensitive to shape of chosen geometry. In many applications, the geometrical configuration is not regularly-shaped and the convective transport rates can be effectively controlled through a vital geometrical parameter arising in non-regular geometries. ${ }^{33,34}$ A detailed review and discussion on various constraints affecting nanofluid flow behavior and heat dissipation rate in different geometries and passages have been reported. ${ }^{35,36}$

In many practical situations, such as thermal bridge or heat barrier or design of thermal insulation, the impact of wall thermal conduction should be taken into consideration, otherwise leads to inaccuracies in the prediction of flow movement and thermal behavior. Therefore, the impact of wall conduction on buoyant fluid motion and 
associated thermal removal from the hot boundary have received substantial attention by many theoretical and experimental analysis. In this direction, one of the pioneering and detailed study was made by Kaminski and Prakash $^{37}$ in a square geometry by considering three different wall conduction models. For larger thermal gradients, they observed asymmetric flow field and non-uniformity in temperature along the interface between solid and fluid. Ben-Nakhi and Chamkha ${ }^{38}$ performed numerical analysis to understand the influence of a slim tilted baffle on conjugate buoyant convective motion in a square geometry having finite thickness on three boundaries. In a 2D square section, with thickness on its side wall, the change in flow motion and thermal behavior of liquid gallium subjected to an externally imposed tilted magnetic force and wall conductivity was examined by Belazizia et al. ${ }^{39}$ and reported that magnetic angle is a crucial parameter in controlling thermal transport. Conjugate buoyant motion in finite shaped geometry containing porous materials have also been investigated by many researchers using different porous media models ${ }^{40,41}$ by considering uniform and discrete heating.

Conjugate buoyant motion and transport due to the presence of a solid block in an inclined ${ }^{42}$ and non-inclined ${ }^{43,44}$ square geometry was numerically analyzed in the presence and absence of magnetic field. By adopting Buongiorno's model, Sheremet and Pop ${ }^{45}$ analyzed the Brownian movement and thermophoresis effect of nanofluid for a vast range of critical parameters and determined the range of parameters at which the chosen model could be used. Alsabery et al. ${ }^{46}$ discussed the impacts of non-uniform thermal conditions on nanofluid buoyant motion in a square geometry with thick bottom wall. The conjugate flow and thermal behavior of nanofluids undergo predominant changes due to the presence of protruding source or block in a finite geometry. ${ }^{47,48}$ Ghalambaz et al. ${ }^{49}$ addressed the conjugate buoyant motion and thermal transport of hybrid nanofluid in a square geometry and discussed the impacts of all key parameters. Reddy and Narasimham ${ }^{50}$ performed numerical simulations to study combined conduction-convection in an enclosed annular region between inner heated rod and an outer cylinder for vast ranges of thermal conductivity ratios and presented thermal transfer correlations. Later, conjugate buoyant transport in a porous material placed in the annular region between two solid cylinders was numerically analyzed by Badruddin et al. ${ }^{51}$ Recently, John et al. ${ }^{52}$ made a detailed review on conjugate thermal transfer analysis covering various applied and theoretical aspects.

We made a meticulous and systematic literature survey on conjugate buoyant convection of clear fluids as well as nanofluids in all shapes of finite geometries by considering most of the additional constraints. From the detailed survey of theoretical and experimental investigations, we have noticed that the conjugate buoyant motion and thermal transport behavior of nanofluids inside the enclosed annular region has not been analyzed so far. Therefore, keeping the applications involving thermal bridge or heat barrier in mind, a detailed numerical investigation is being carried out to explore the impacts of various key parameters on flow and thermal behavior of three different nanofluids in the annular geometry having finite thickness at the inner cylinder.

\section{Formulation of problem \& Numerical procedure}

Formulation of problem. The geometrical structure, as portrayed in Fig. 1, is the enclosed concentric annulus region between two upright cylindrical tubes with solid inner cylinder of thickness $(\varepsilon)$. The thermal conditions along the vertical boundaries are maintained such that the inner boundary is hotter than the outer surface, while at the horizontal surfaces, adiabatic condition is imposed. The annular gap is occupied by the water-based nanofluids having different nanoparticles, such as aluminum oxide $\left(\mathrm{Al}_{2} \mathrm{O}_{3}\right)$ or titanium oxide $\left(\mathrm{TiO}_{2}\right)$ or copper $(\mathrm{Cu})$. It is assumed that the water and nanoparticles are in thermal equilibrium. Thermo-physical properties of water and nanoparticles are given in Table 1. Also, the flow is considered to be laminar, two-dimensional and axisymmetric. The Boussinesq approximation which accounts the density variation in the body force term and constant elsewhere of momentum equation is utilized in this analysis. Further, the solid and fluid thermal conductivities are considered to be different. By utilizing the above postulates, the governing equations are: 


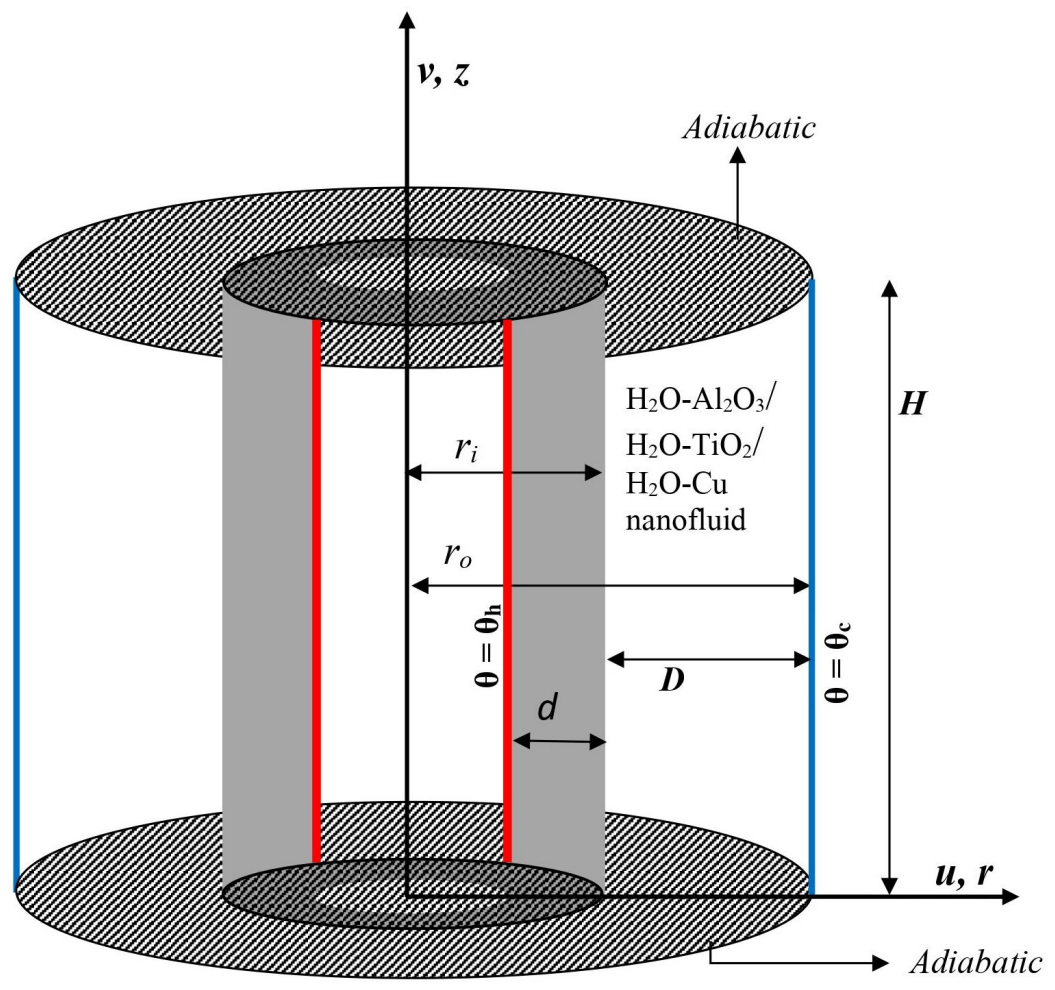

Figure 1. The annular geometry, Coordinate system and thermal conditions

$$
\begin{aligned}
\nabla \cdot \vec{q} & =0, \\
\rho_{n f}\left[\frac{\partial \vec{q}}{\partial t^{*}}+(\vec{q} \cdot \nabla) \vec{q}\right] & =-\nabla p+\mu_{n f} \nabla^{2} \vec{q}+(\rho \beta)_{n f} \vec{g}\left(\theta_{n f}-\theta_{c}\right), \\
\frac{\partial \theta_{n f}}{\partial t^{*}}+(\vec{q} \cdot \nabla) \theta_{n f} & =\alpha_{n f} \nabla^{2} \theta_{n f}, \\
\frac{\partial \theta_{w}}{\partial t^{*}} & =\alpha_{w} \nabla^{2} \theta_{w} .
\end{aligned}
$$

In the present study, the thermophysical properties of nanofluid are chosen using below relations: ${ }^{17}$

$$
\begin{aligned}
\rho_{n f} & =(1-\phi) \rho_{f}+\phi \rho_{p}, \\
(\rho \beta)_{n f} & =(1-\phi)(\rho \beta)_{f}+\phi(\rho \beta)_{p} \\
\left(\rho C_{p}\right)_{n f} & =(1-\phi)\left(\rho C_{p}\right)_{f}+\phi\left(\rho C_{p}\right)_{p} \\
\frac{k_{n f}}{k_{f}} & =\frac{k_{p}+2 k_{f}-2 \phi\left(k_{f}-k_{p}\right)}{k_{p}+2 k_{f}+\phi\left(k_{f}-k_{p}\right)} \\
\mu_{n f} & =\frac{\mu_{f}}{(1-\phi)^{2.5}} \\
\alpha_{n f} & =\frac{k_{n f}}{\left(\rho C_{p}\right)_{n f}} .
\end{aligned}
$$

In these expressions, the subscripts $n f, f$ and $p$ represents the nanofluid, fluid and nanoparticle, respectively. Applying the following transformations, 
Table 1. Thermo-physical properties of water and nanoparticles. ${ }^{27}$

\begin{tabular}{lllll}
\hline Property & $\mathrm{H}_{2} \mathrm{O}$ & $\mathrm{Cu}$ & $\mathrm{Al}_{2} \mathrm{O}_{3}$ & $\mathrm{TiO}_{2}$ \\
\hline$\rho\left(\mathrm{kg} / \mathrm{m}^{3}\right)$ & 997.1 & 8933 & 3970 & 4250 \\
$C_{p}(\mathrm{~J} / \mathrm{kg} \mathrm{K})$ & 4179 & 385 & 765 & 686.2 \\
$k(\mathrm{~W} / \mathrm{mK})$ & 0.613 & 400 & 40 & 8.9538 \\
$\beta\left(\mathrm{K}^{-1}\right)$ & $21 \times 10^{-5}$ & $1.67 \times 10^{-5}$ & $0.85 \times 10^{-5}$ & $0.9 \times 10^{-5}$ \\
\hline
\end{tabular}

$(R, Z)=\frac{(r, z)}{D}, \quad \varepsilon=\frac{d}{D}, \quad U=\frac{u D}{\alpha_{f}}, \quad V=\frac{v D}{\alpha_{f}}, \quad t=\frac{t^{*}}{\left(D^{2} / \alpha_{f}\right)}, \quad T_{w}=\frac{\left(\theta_{w}-\theta_{c}\right)}{\left(\theta_{h}-\theta_{c}\right)}, \quad T_{n f}=\frac{\left(\theta_{n f}-\theta_{c}\right)}{\left(\theta_{h}-\theta_{c}\right)}$, $P=\frac{p}{\rho_{n f}\left(\alpha_{f} / D\right)^{2}}$,

we finally get the dimensionless governing equations:

$$
\begin{aligned}
\frac{\partial U}{\partial R}+\frac{\partial V}{\partial Z}+\frac{U}{R} & =0 \\
\frac{\partial U}{\partial t}+U \frac{\partial U}{\partial R}+V \frac{\partial U}{\partial Z} & =-\frac{\partial P}{\partial R}+\frac{\mu_{n f}}{\rho_{n f} \alpha_{f}}\left[\frac{\partial^{2} U}{\partial R^{2}}+\frac{1}{R} \frac{\partial U}{\partial R}+\frac{\partial^{2} U}{\partial Z^{2}}-\frac{U}{R^{2}}\right] \\
\frac{\partial V}{\partial t}+U \frac{\partial V}{\partial R}+V \frac{\partial V}{\partial Z} & =-\frac{\partial P}{\partial Z}+\frac{\mu_{n f}}{\rho_{n f} \alpha_{f}}\left[\frac{\partial^{2} V}{\partial R^{2}}+\frac{1}{R} \frac{\partial V}{\partial R}+\frac{\partial^{2} V}{\partial Z^{2}}\right]+\frac{(\rho \beta)_{n f}}{\rho_{n f} \beta_{f}} \operatorname{Ra} \operatorname{Pr} T_{n f}, \\
\frac{\partial T_{n f}}{\partial t}+U \frac{\partial T_{n f}}{\partial R}+V \frac{\partial T_{n f}}{\partial Z} & =\frac{\alpha_{n f}}{\alpha_{f}}\left[\frac{\partial^{2} T_{n f}}{\partial R^{2}}+\frac{1}{R} \frac{\partial T_{n f}}{\partial R}+\frac{\partial^{2} T_{n f}}{\partial Z^{2}}\right] \\
\frac{\partial T_{w}}{\partial t} & =\frac{\alpha_{w}}{\alpha_{f}}\left[\frac{\partial^{2} T_{w}}{\partial R^{2}}+\frac{1}{R} \frac{\partial T_{w}}{\partial R}+\frac{\partial^{2} T_{w}}{\partial Z^{2}}\right]
\end{aligned}
$$

where $R a=\frac{g \beta_{f} \Delta \theta D^{3}}{v_{f} \alpha_{f}}$ and $\operatorname{Pr}=\frac{v_{f}}{\alpha_{f}}$ are the Rayleigh and Prandtl numbers, respectively.

By introducing two-dimensional stream function $\psi(R, Z)$, the momentum equations (6) and (7) can be expressed in the following vorticity-stream function form:

$$
\begin{aligned}
\frac{\partial \zeta}{\partial t}+U \frac{\partial \zeta}{\partial R}+V \frac{\partial \zeta}{\partial Z}-\frac{U \zeta}{R} & =\frac{\mu_{n f}}{\rho_{n f} \alpha_{f}}\left[\nabla^{2} \zeta-\frac{\zeta}{R^{2}}\right]-\frac{(\rho \beta)_{n f}}{\rho_{n f} \beta_{f}} \operatorname{Ra} \operatorname{Pr} \frac{\partial T_{n f}}{\partial R} \\
\zeta & =\frac{1}{R}\left[\frac{\partial^{2} \psi}{\partial R^{2}}-\frac{1}{R} \frac{\partial \psi}{\partial R}+\frac{\partial^{2} \psi}{\partial Z^{2}}\right] \\
U & =\frac{1}{R} \frac{\partial \psi}{\partial Z}, \quad V=-\frac{1}{R} \frac{\partial \psi}{\partial R}
\end{aligned}
$$

where $\nabla^{2}=\frac{\partial^{2}}{\partial R^{2}}+\frac{1}{R} \frac{\partial}{\partial R}+\frac{\partial^{2}}{\partial Z^{2}}$. 
The initial and boundary conditions in dimensionless form are;

$$
\begin{aligned}
t=0: U & =V=T_{w}=T_{n f}=0, \psi=\zeta=0 ; \quad \text { at } \quad 0 \leq Z \leq A \quad \text { and } \quad \frac{1}{\lambda-1} \leq R \leq \frac{\lambda}{\lambda-1} . \\
t>0: \psi & =\frac{\partial \psi}{\partial R}=0, T_{w}=1 \quad \text { at } \quad R=\frac{1}{\lambda-1} \quad \text { and } \quad 0 \leq Z \leq A \\
\psi & =\frac{\partial \psi}{\partial R}=0, T_{n f}=0 \quad \text { at } \quad R=\frac{\lambda}{\lambda-1} \quad \text { and } \quad 0 \leq Z \leq A \\
\psi & =\frac{\partial \psi}{\partial Z}=0, \frac{\partial T_{w}}{\partial Z}=\frac{\partial T_{n f}}{\partial Z}=0 \quad \text { at } \quad Z=0 \quad \text { and } \quad Z=A \\
\frac{\partial T_{n f}}{\partial R} & =K r \frac{\partial T_{w}}{\partial R} \text { at the interface, }
\end{aligned}
$$

where $K r=\frac{k_{w}}{k_{n f}}$ is the thermal conductivity ratio.

The thermal dissipation rates are measured through the average Nusselt numbers along the solid wall $\left(\overline{N u}_{w}\right)$ as well as at the interface $\left(\overline{N u}_{i}\right)$ and are given by

$$
\overline{N u}_{w}=\frac{1}{A} \int_{0}^{A} N u_{w} d Z \text { and } \overline{N u}_{i}=\frac{1}{A} \int_{0}^{A} N u_{i} d Z
$$

where $N u_{w}=-\left(\frac{\partial T}{\partial R}\right)_{R=\frac{1}{\lambda-1}}$ and $N u_{i}=-\frac{k_{n f}}{k_{f}}\left(\frac{\partial T}{\partial R}\right)_{R=\varepsilon}$.

Numerical methodology. The coupled and nonlinear PDEs and associated supplementary conditions governing the physical processes are numerically solved by utilizing suitable implicit finite difference method (FDM). In particular, the transient PDEs, such as vorticity and temperature equations are discretized using Alternating Direction Implicit (ADI) method. Successive Line Over Relaxation (SLOR) method is adopted to solve steady-state stream function equation. These FDM based techniques reduce the PDEs to a system of linear algebraic FD equations with tridiagonal structure and using Tri-diagonal Matrix Algorithm (TDMA), the solutions are obtained. We have performed the grid independence trials by considering five different grid sizes, varying from sparse to finer grids, as shown in Table 2. The global Nusselt numbers along the inner cylinder and interface are measured for each grid size and used as the sensitivity measure to check the optimum grid size. We did not detect a considerable change in $\overline{N u}$ at the wall and interface between $201 \times 201$ and $251 \times 251$ grid sizes. Therefore, by accounting accuracy and computation time, a grid size of $201 \times 201$ is utilized for all simulations of present investigation. An in-house FORTRAN code was written and few verifications are executed to validate the simulation results.

Validation. The current simulations are tested with different benchmark data present in the literature. Firstly, the global Nusselt number for an annular geometry containing $\mathrm{Al}_{2} \mathrm{O}_{3}$ based nanofluid in the limiting case of zero thickness of inner cylinder is compared with present predictions. The relative difference in $\overline{N u}$ between our predictions and those estimated from the correlation suggested by Abouali and Falahatpishesh, ${ }^{15}$ displayed in Table 3 , is in acceptable range for all magnitudes of $R a$. An additional quantitative comparison of flow and thermal contours in a square geometry containing $\mathrm{Al}_{2} \mathrm{O}_{3}$ based nanofluid with $\phi=0.04$ at $\mathrm{Ra}=10^{6}$ is generated by setting $\lambda=1$ in the present analysis. An excellent qualitative comparison of isotherms and streamlines between present and Ho et al. ${ }^{26}$ analysis is exhibited in Fig. 2 and the agreement between the predictions is quite good. The excellent concurrence in the qualitative and quantitative comparison of our results with benchmark predictions for square and annular geometries ensure the credibility of developed code. 
Table 2. Grid independence test for $R a=10^{6}, \varepsilon=0.4, K r=10$ and $\phi=0.2$.

\begin{tabular}{lll}
\hline Grid size & $\overline{N u_{w}}$ & $\overline{N u_{i}}$ \\
\hline $81 \times 81$ & 0.9875297 & 9.0849894 \\
$101 \times 101$ & 0.9902507 & 9.0542851 \\
$161 \times 161$ & 0.9939314 & 9.0221870 \\
$201 \times 201$ & 0.9950641 & 9.0145831 \\
$251 \times 251$ & 0.9952458 & 9.0139465 \\
\hline
\end{tabular}

Table 3. Comparison of $\overline{N u}$ of present study with Abouali and Falahatpishesh. ${ }^{15}$

\begin{tabular}{lllll}
\hline $\begin{array}{l}R a \\
(G r \times P r)\end{array}$ & $\begin{array}{l}\text { Abouali and } \\
\text { Falahatpishesh }{ }^{15}\end{array}$ & Present study & $\begin{array}{l}\text { Relative } \\
\text { difference }(\%)\end{array}$ \\
\hline $6 \times 10^{3}$ & 2.8673 & 2.8843 & 0.59 & $\phi=0.00$ \\
& 2.7291 & 2.7401 & 0.40 & $\phi=0.02$ \\
& & & & \\
$6 \times 10^{4}$ & 5.4385 & 5.4796 & 0.75 & $\phi=0.00$ \\
& 5.1762 & 5.1836 & 0.14 & $\phi=0.02$ \\
$6 \times 10^{5}$ & 10.3150 & 10.4015 & 0.83 & $\phi=0.00$ \\
& 9.8178 & 9.9132 & 0.96 & $\phi=0.02$ \\
\hline
\end{tabular}

\section{Results and discussion}

In the present analysis, the combined conduction-convective flow and associated thermal transport analysis is performed in the solid and annular regions. The dimensionless parameters arising in this investigation, varied over wide spectrum of values, are the wall thickness $(0.1 \leq \varepsilon \leq 0.5)$, thermal conductivity ratio $(0.1 \leq K r \leq 10)$, Rayleigh number $\left(10^{3} \leq R a \leq 10^{6}\right)$ and the nanoparticle volume fraction $(0 \leq \phi \leq 0.2)$. For all simulations, the values of Prandtl number and radius ratio are fixed at $P r=6.2$ and $\lambda=2$ respectively. The impact of these parameters on buoyant motion of three different nanofluids in the annular domain has been investigated qualitatively and quantitatively. The simulations are displayed through the flow and thermal contours, temperature profiles and global thermal dissipation rates.

Figures 3-5 represent the impacts of $R a$ on flow and thermal contours for three different nanofluids and base-fluid by keeping $K r=5$ and $\varepsilon=0.2$. For lower magnitude of $R a\left(R a=10^{4}\right)$, the conventional single-eddy flow structure with moderate circulation rate is noticed and slightly distorted isothermal contours with less stratification indicates conduction-dominated heat transfer with minimal convective transport. With an increase in $R a$ to the maximum chosen value $\left(R a=10^{6}\right)$, a 3 -fold enhancement in the strength of nanofluid buoyant motion is observed. The streamline vortex pattern has been modified with an enhanced value of $R a=10^{6}$. Flow and thermal contour pattern for all three different nanofluids, considered in this simulations, have not changed appreciably. However, though the streamlines appear to be similar for all three nanofluids, a meager change in fluid flow strength is detected. The less-distorted isotherms become well distorted and stratified with a rise in magnitude of $R a$ which implies that the mode of thermal transport has moved from conduction to convection dominated state. At higher $R a$, the isotherms 

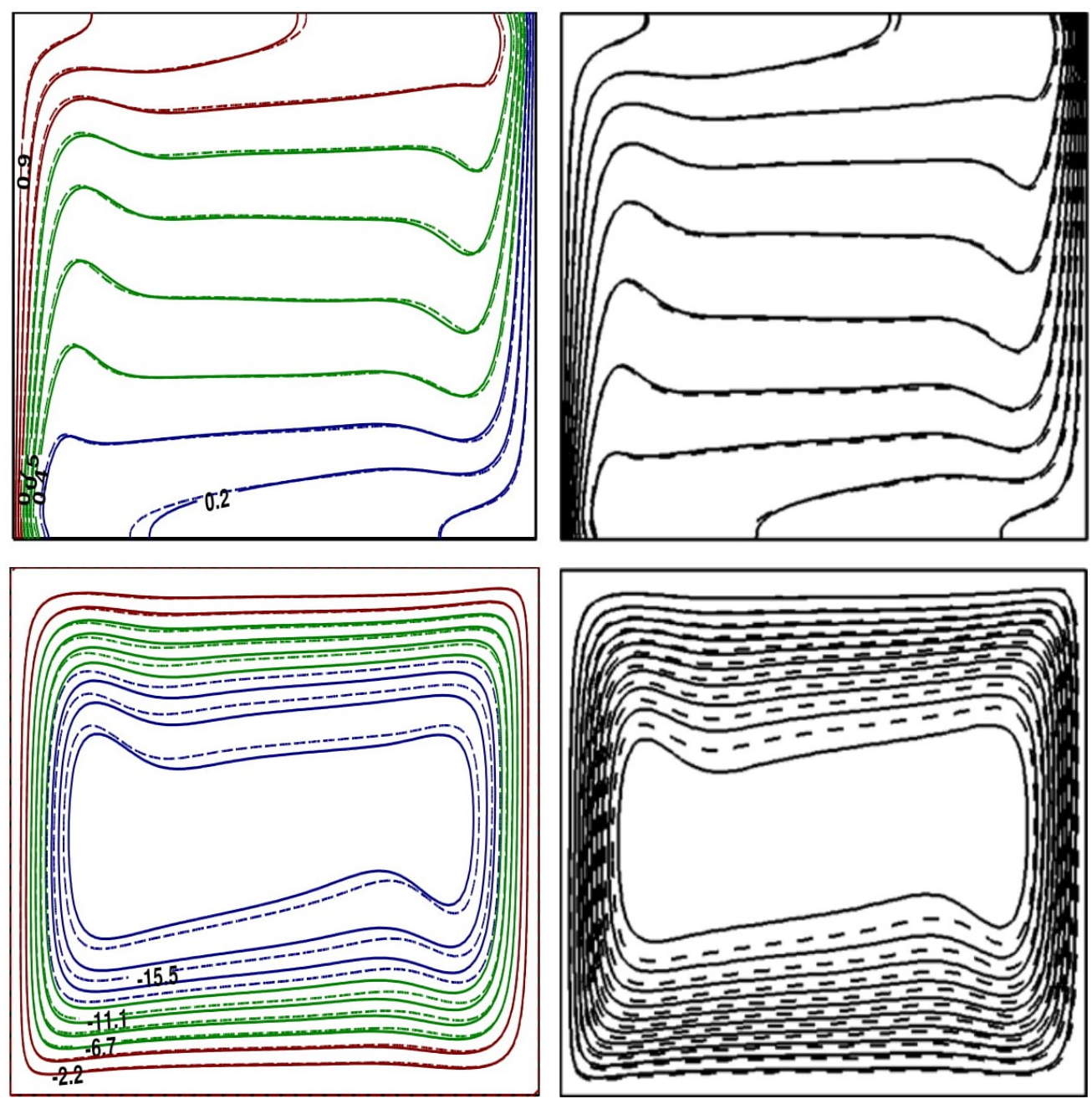

Figure 2. Comparision of present simulations (left) with the results of Ho et al. ${ }^{26}$ (right). Base fluid (dotted line) and nanofluid (solid line). (color online)

are horizontally skewed in the fluid region due to convection-dominant state and larger thermal gradients are noticed near the solid-fluid interface. Similar isothermal structure pattern is detected for all three nanofluids and base fluid.

In Figs. 6-8, solid, dotted and dashed curves represent $\mathrm{Al}_{2} \mathrm{O}_{3}, \mathrm{TiO}_{2}$ and $\mathrm{Cu}$-nanofluids respectively. Since the analysis involves fluid region along with solid portion, the impact of thermal conductivities of both solid and nanofluid need to be considered. The impact of conductivity ratio $(K r)$ on streamlines and isotherms is depicted in Fig. 6 for all three nanofluids. For poor thermal conductivity of the wall $(K r=0.1)$, the solid wall acts like an insulated material that results in less thermal dissipation through the wall. Hence, the temperature difference between the solid-fluid interface and cold outer cylindrical surface is moderate, due to which the amount of heat transferred to the fluid region is very minimal (see Fig. 6). For lower thermal conductivity ratio, the isotherms are confined mainly to the solid wall due to the inability of wall thermal conductivity. As $K r$ increases, the isotherms are near stratified state and the flow pattern also changes. For $K r=10$, the thermal gradient in solid cylinder is very minimal and the solid wall temperature is nearly identical with hot wall temperature due to higher wall thermal conductivity. Further, at higher magnitude of $K r$, a strong temperature stratification exists and the buoyant nanofluid motion reveals an enhanced flow strength.

The influence of solid wall thickness on buoyant flow and thermal contours is presented in Fig. 7 for $R a=10^{6}$ by considering all three nanofluids. For $\varepsilon=0.1$, heat is transferred very rapidly from solid cylinder to nanofluid 

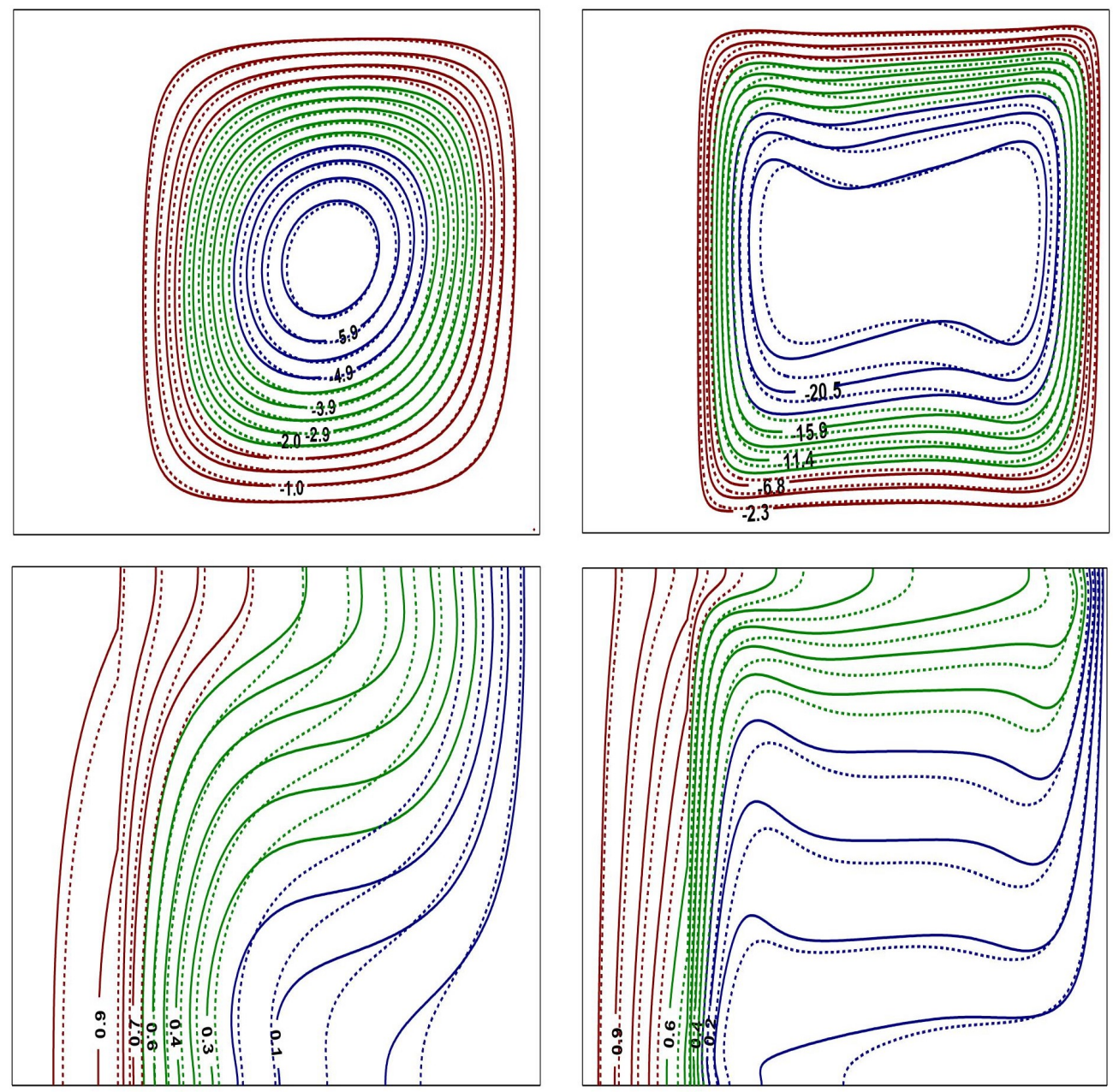

Figure 3. Impact of $R a$ on flow and thermal contours at $R a=10^{4}$ (left) and $R a=10^{6}$ (right) for base fluid (solid curve) and $\mathrm{Al}_{2} \mathrm{O}_{3}$-nanofluid (dotted curve) with $\phi=0.1, \mathrm{Kr}=5$ and $\varepsilon=0.2$. (color online)

region due to lower thermal resistance of the wall. Thus, heat transfer is mainly dominated by convection and isotherms are found to be more stratified with strong flow circulation strength. As the wall thickness is increased, the thermal resistance of the wall also increases leading to retardation of heat dissipation through the wall. As a result, the temperature difference between the solid-fluid interface and cold right wall has been reduced which causes the reduction of flow circulation strength. The thermal stratification, identified from the isotherm contours, also profoundly disturbed with an increase in inner cylinder thickness. The thermal contours have spread over the solid cylindrical region indicating a significant reduction of thermal gradients in the solid wall as $50 \%$ of annular domain becomes solid $(\varepsilon=0.5)$. As a result, the thermal energy available at the interface may not be sufficient to enhance the fluid flow strength and can be witnessed from the extreme stream function values.

The variation in buoyant flow and thermal structure due to nanoparticle concentration is discussed in Fig. 8. By increasing the nanoparticle concentration, the thermal conductivity of the nanofluids increase and that leads to an enhancement in the fluid flow intensity. As noticed from streamlines, the flow pattern is not sensitive to the fractional quantity of nanoparticles considered in the study. Further, for lower nanoparticle volume concentration, the flow and thermal structure remains unchanged irrespective of the type of nanoparticle utilized in this investigation. However, a further increase in $\phi$ produces a minor variation of streamline and isotherm contours for different nanofluids considered for the analysis.

The thermal dissipation rate from the annular boundary to interface and in turn to the surrounding nanofluids 

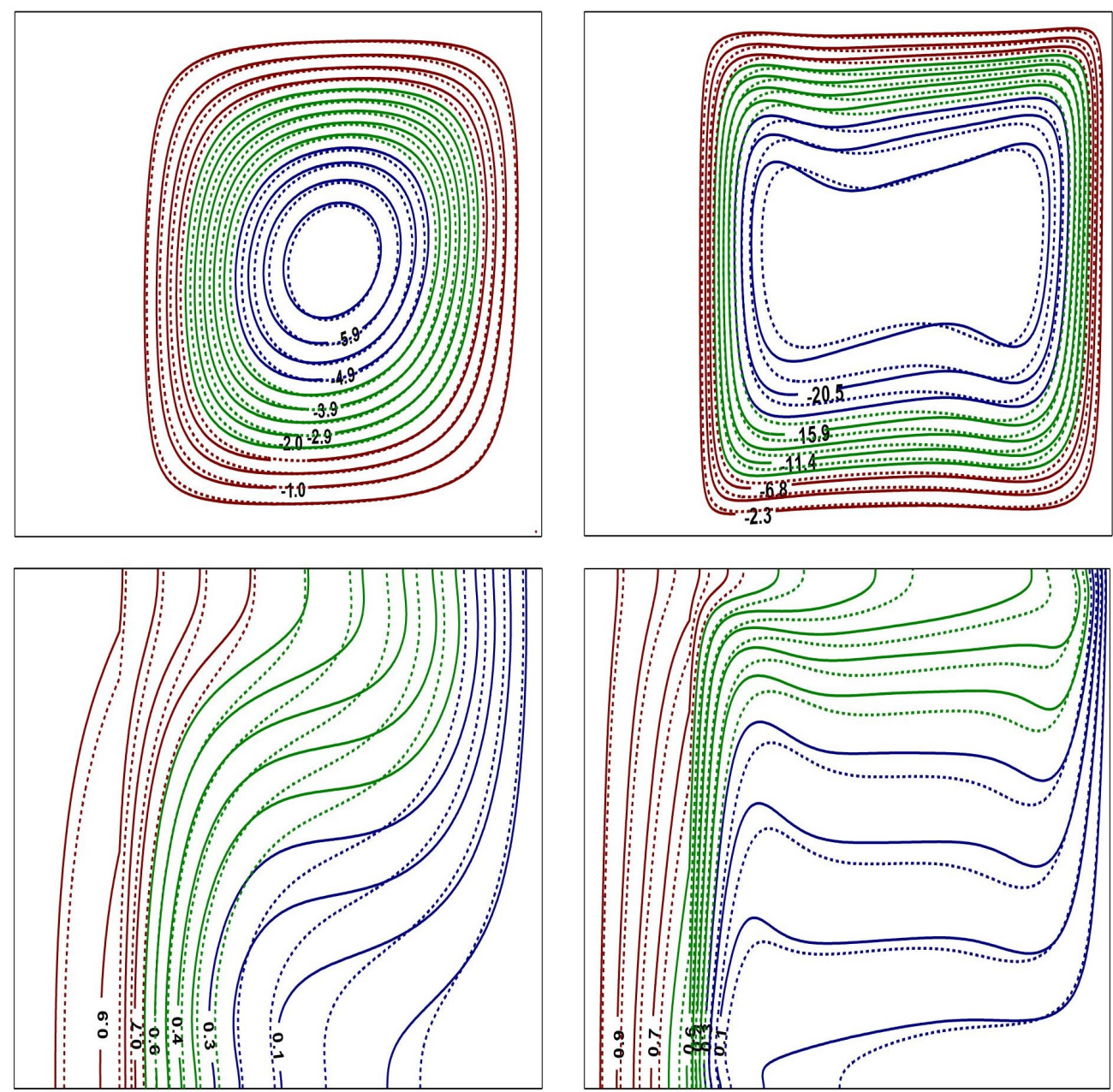

Figure 4. Impact of $R a$ on flow and thermal contours at $R a=10^{4}$ (left) and $R a=10^{6}$ (right) for base fluid (solid curve) and $\mathrm{TiO}_{2}$-nanofluid (dotted curve) with $\phi=0.1, \mathrm{Kr}=5$ and $\varepsilon=0.2$. (color online)

is highly influenced by various parameters such as $R a, K r, \varepsilon$ and $\phi$. The effect of wide range of these parameters on the global heat dissipation rate at the solid cylinder and interface of the annulus is displayed in Figs. 9-13. The effects of $R a$ and $K r$ on overall thermal transport rate at the wall and interface are presented in Fig. 9 for three nanofluids. In general, the enhancement of thermal dissipation rate strongly depends on the increment in Rayleigh number caused by increasing the temperature difference or altering the value of $K r$. For all three nanofluids, the average $N u$ at the wall is higher at low conductivity ratio and lower for larger value of $K r$. Though the nanofluid occupied in annular region is not in direct contact with the hot inner wall, the global dissipation rate at solid boundary is slightly influenced by the choice of nanofluid. This can be anticipated from the thermal condition imposed at the interface, which carries the influence of nanoparticle used. On contrast, the heat transport rate at the interface strongly depends on the choice of nanoparticle. Further, among the three nanoparticles used in the analysis, $\mathrm{Cu}$-based nanofluid produces enhanced heat dissipation as compared to $\mathrm{Al}_{2} \mathrm{O}_{3}$ and $\mathrm{TiO}_{2}$ nanoparticles. In general, the global interface $N u$ enhances with an increment in $R a$ at all conductivity ratios except for $K r=0.1$, at which $\overline{N u_{i}}$ remains invariant with $R a$. For $K r=0.1$, the wall conductivity is minimum compared to that of nanofluid and hence the solid wall behaves like an insulated material. Therefore, for $K r=0.1$, the change in temperature difference between the vertical walls has not influenced the global heat transport rate at the interface for all nanofluids considered in the current investigation.

Figure 10 exhibits the influence of wall thickness and Rayleigh number on overall heat transport rate at the 

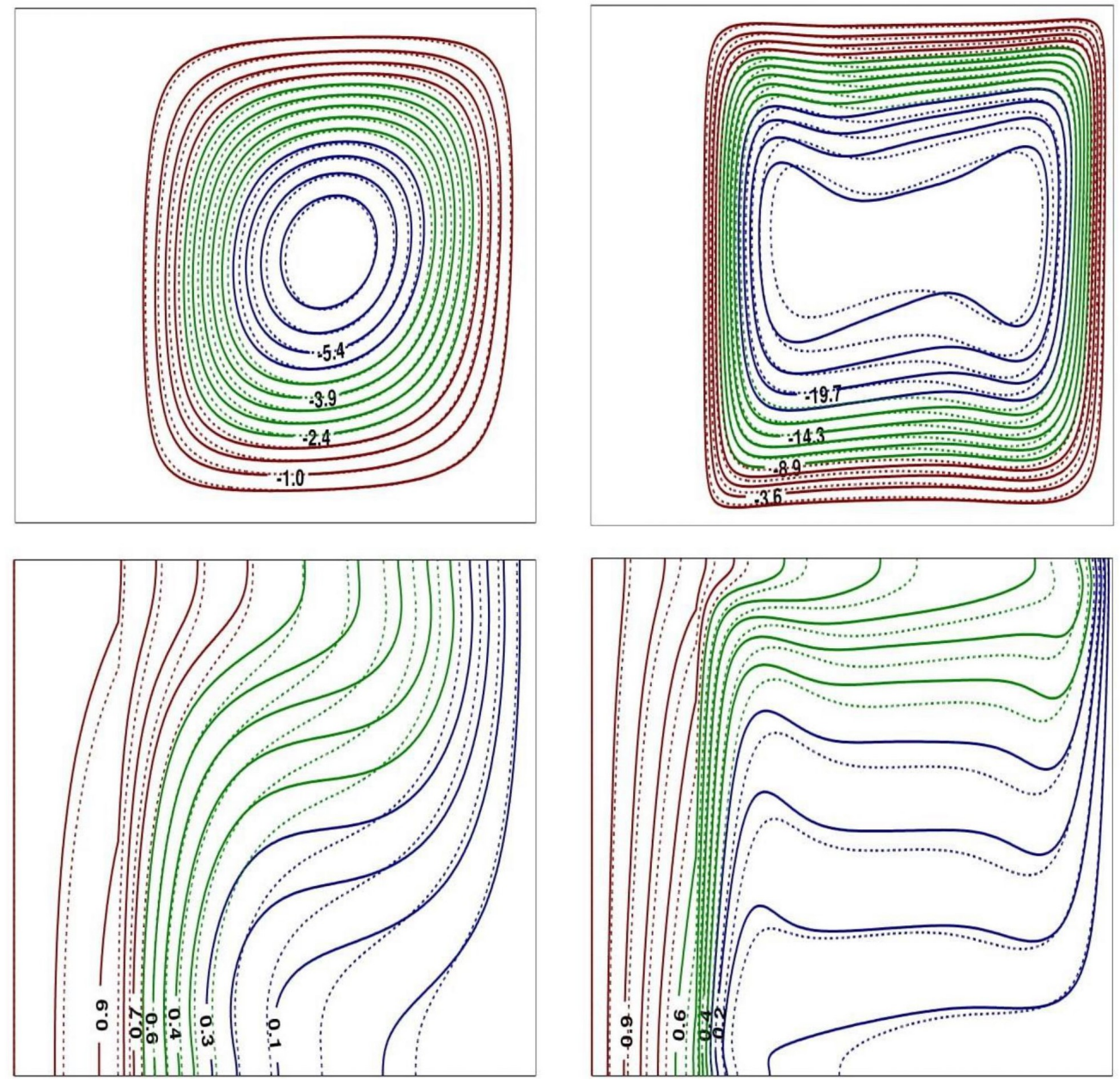

Figure 5. Impact of $R a$ on flow and thermal contours at $R a=10^{4}$ (left) and $R a=10^{6}$ (right) for base fluid (solid curve) and $C u$-nanofluid (dotted curve) with $\phi=0.1, K r=5$ and $\varepsilon=0.2$. (color online)

wall and interface for three different nanofluids with $\phi=0.1$ and $K r=2$. The impact of wall thickness on thermal dissipation from the wall strongly depends on the value of $R a$. For lower $R a$, the heat transfer at the wall increases with an increase in wall thickness. However, at higher Rayleigh number, the heat transfer at the wall declines with an increase in the wall thickness. Also, a sharp increase in $\overline{N u}$ with $R a$ can be noticed at lower wall thickness $(\varepsilon=0.1)$ and further increment in $\varepsilon$ reveals meager variation of thermal transport with $R a$. As discussed earlier, the impacts of different nanofluids on wall $\overline{N u}$ is indistinguishable. As regards to thermal dissipation rate at the interface, $\overline{N u_{i}}$ enhances with an increase in $R a$ and reducing the wall thickness $(\varepsilon)$, in general. For lower $R a$, heat transport at the interface does not show any appreciable variation for all three nanofluids. By increasing $R a$, the thermal transport rate increases and a marginal increase in the average Nusselt number is observed for $\mathrm{Cu}$-water nanofluid followed by $\mathrm{Al}_{2} \mathrm{O}_{3}$-water and $\mathrm{TiO}_{2}$-water nanofluids for all values of $\mathrm{Ra}$ and $\varepsilon$. An increase in wall thickness leads to the enhancement of conductive resistance in the solid wall and this results in retardation of the average interface Nusselt number.

Figure 11 deals with the effect of $R a$ and $\phi$ on overall heat dissipation rate at the wall and interface for different nanofluids for $K r=2$ and $\varepsilon=0.2$. An increase in nanoparticle volume fraction leads to suppress the overall heat dissipation from the wall, but thermal transport enhancement is observed with an increase in $R a$. For lower nanoparticle concentrations $(\phi \leq 0.1)$, the influence of nanoparticles on average wall Nusselt number is insignificant. However, for nanofluids with higher nanoparticle concentration, minor variation in $\overline{N u_{w}}$ can be observed for 

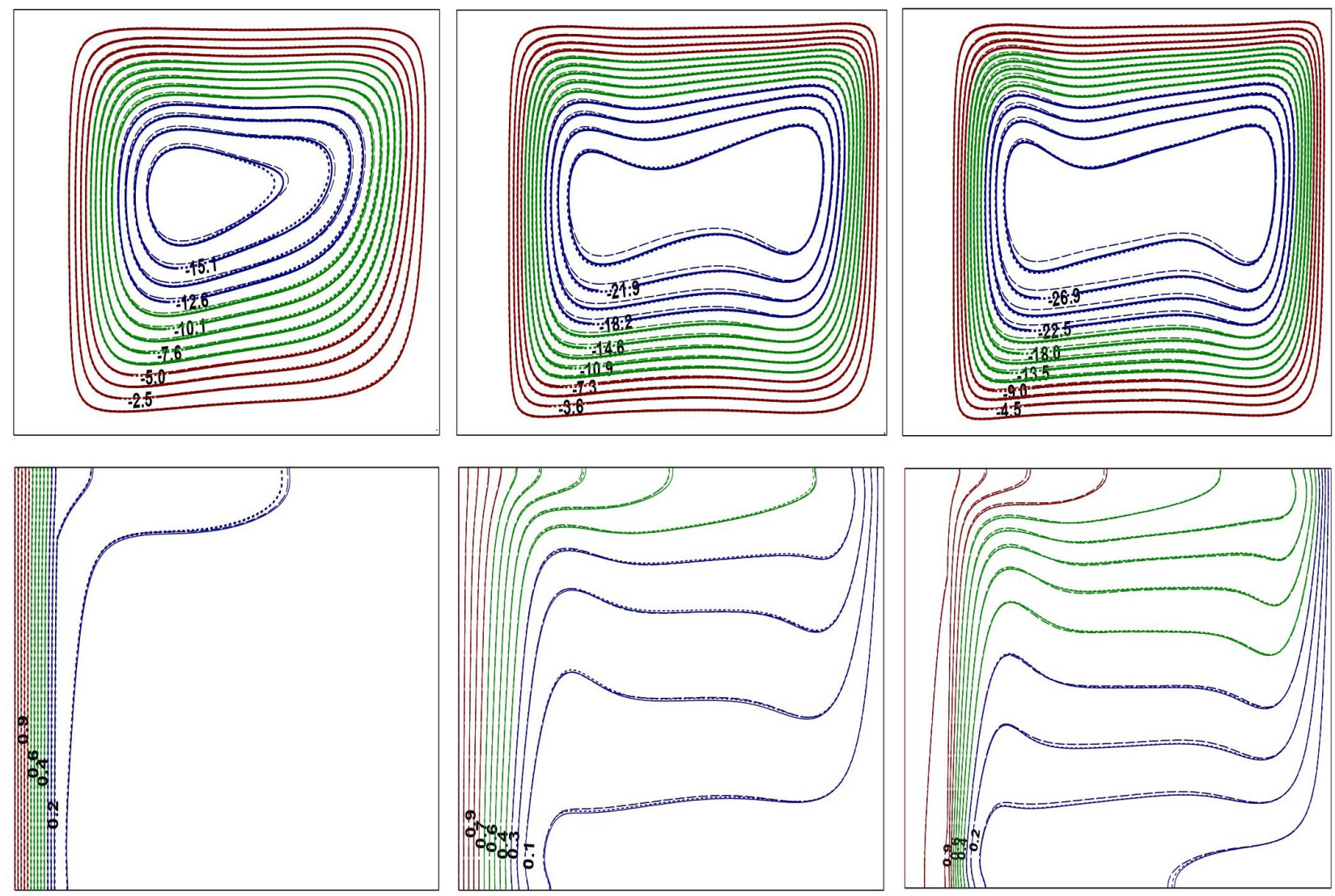

Figure 6. Impact of $K r$ on flow and thermal contours at $R a=10^{6}, \phi=0.1$ and $\varepsilon=0.1$. $\mathrm{Kr}=0.1$ (left), $\mathrm{Kr}=1.0$ (center) and $\mathrm{Kr}=10$ (right). (color online)

$\mathrm{Cu} \& \mathrm{Al}_{2} \mathrm{O}_{3}$-water nanofluids contributing to a slightly higher heat transport rate. The overall thermal dissipation rate along the interface has been enhanced as the magnitudes of $R a$ and $\phi$ increases. At higher values of $R a$, due to enhanced temperature difference, substantial amount of thermal transfer occurs between inner hot and outer cold cylinders. As a result, heat transfer rate at the interface is comparatively higher than lower Rayleigh numbers. A similar prediction has been observed for the three different nanofluids considered in our analysis. Also, on increasing the percentage of nanoparticle dispersed in the base fluid, the thermal conductivity of nanofluid is enhanced and with further addition of nanoparticles, the heat removal rate at the interface is further enhanced for all values of $R a$. The estimated global heat transport rate for the nanofluids with three different nanoparticles, such as $\mathrm{Cu}, \mathrm{Al}_{2} \mathrm{O}_{3}$ and $\mathrm{TiO}_{2}$ reveals that $\mathrm{Cu}$-water nanofluid contributes to better enhancement of heat transport for all values of $\mathrm{Ra}$ and $\phi$. This may be attributed to the fact that copper nanoparticle has comparatively higher thermal conductivity among other chosen nanoparticles. Interestingly, the impact of nanoparticle in enhancing thermal dissipation from interface to adjacent nanofluid is significantly visible at higher magnitudes of $R a$. The meager change in thermal dissipation from the wall with different nanofluids could be anticipated from the influence of nanoparticle through the solid-fluid interface. Also, the impact of nanoparticle on $\overline{N u_{w}}$ is consistent with their influence on thermal dissipation along the interface.

The influence of conductivity ratio and wall thickness on the average Nusselt number at the wall and interface are depicted in Fig. 12. An increase in conductivity ratio or wall thickness produces a sharp decline in the wall heat transfer rate. In general, the thermal dissipation from the wall is a decreasing function of $K r$ and $\varepsilon$. For high conductivity ratio $K r=10$, the influence of wall thickness on thermal transport rate at the wall is insignificant. Also, the impact of different nanofluid on the heat transport from wall is negligible. However, on contrary, the choice of 

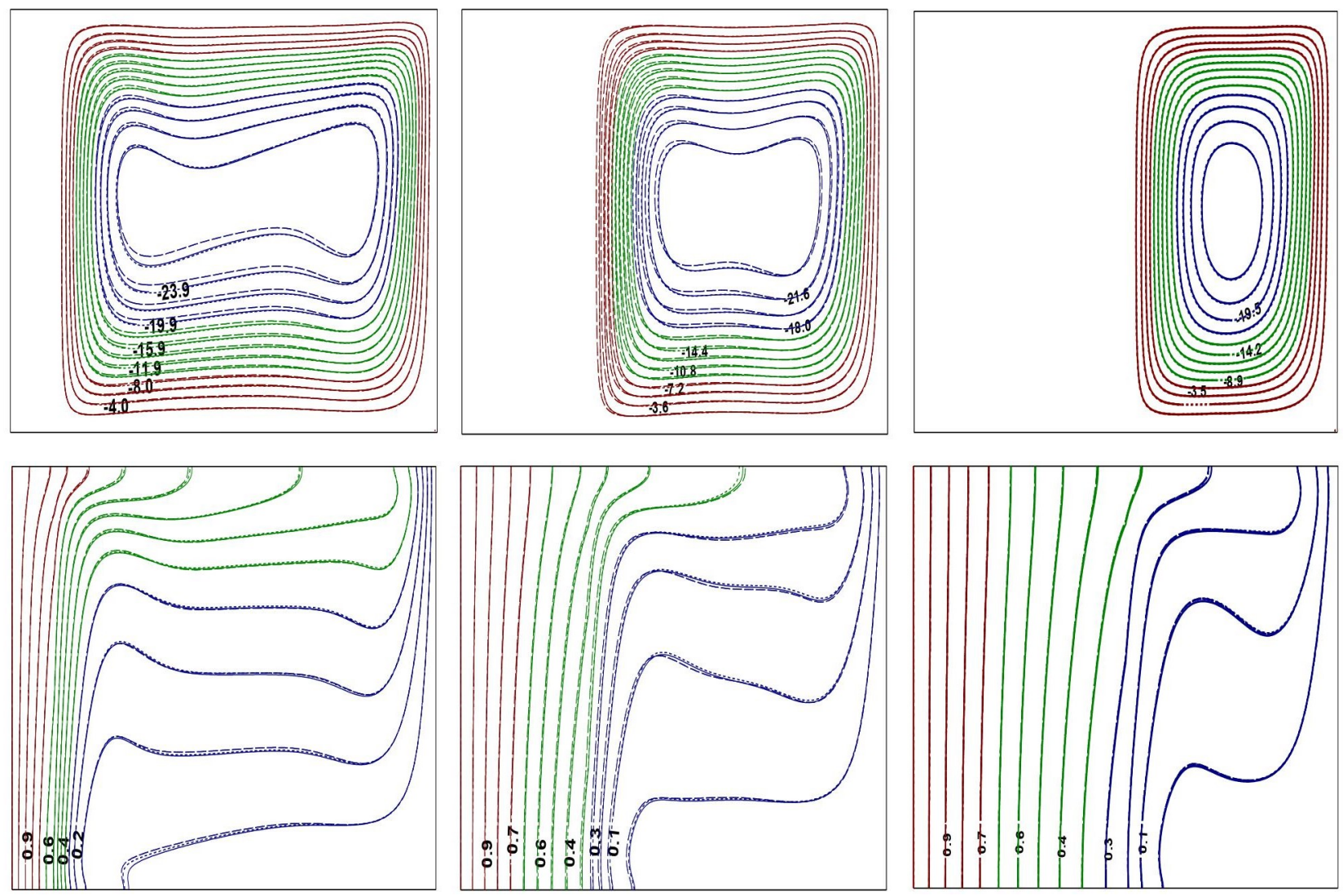

Figure 7. Impact of $\varepsilon$ on flow and thermal contours at $R a=10^{6}, \phi=0.1$ and $K r=2$. $\varepsilon=0.1$ (left), $\varepsilon=0.3$ (center) and $\varepsilon=0.5$ (right). (color online)

$K r, \varepsilon$ and the type of nanofluids on thermal dissipation from the interface has reverse impact. The heat dissipation rate at the interface increases sharply for $K r \leq 5$ and moderate enhancement has been noticed for higher thermal conductivity ratios. The overall heat transport rate at the interface is an increasing function of $\mathrm{Kr}$, but decreases with $\varepsilon$. This is due to the decline in thermal resistance with an increase in $K r$ and decrease in wall thickness. It is noticed that, $\mathrm{Cu}$-water provides maximum heat transport compared to other nanofluids. This can be inferred from the fact that higher thermal conductivity of copper clearly influences the overall heat dissipation rate. Interestingly, the change in $K r$ has different influences on the thermal dissipation rate along the wall and interface. The amount of thermal dissipation from the wall is found to be on higher scale as compared to interface for $K r \leq 1$, however, for $K r>1$, the interface thermal transport is found to be higher than cylindrical wall.

The influence of nanoparticle concentration and solid wall thickness on overall heat transfer rate at the wall and interface are depicted through Fig. 13. In general, an increase in $\varepsilon$ leads to decrease in $\overline{N u}$ due to enhanced conductive resistance in the wall. An increase in wall thickness leads to retardation of thermal transport to the fluid region. Thus, the average $\mathrm{Nu}$ at the solid cylinder and interface are decreasing functions of wall thickness. The impact of nanofluids, pass through the interface, on $\overline{N u_{w}}$ is profoundly influenced by the thickness of inner cylinder. For thick cylindrical wall, negligible change in thermal dissipation is observed for different nanofluids. But, a meager variation is noted for the inner cylinder having comparatively lower thickness. This could be explained from the fact that the infiltration influence of nanofluid through the interface is lowered as wall thickness increases. Further, an increase in nanoparticle concentration results in decline of wall heat transport rate for both thick as well as thinner walls. However, at the interface, an increasing trend is observed with an increase in nanoparticle volume fraction due to enhanced thermal conductivity by the addition of nanoparticles. An in-depth observation from the variation of 

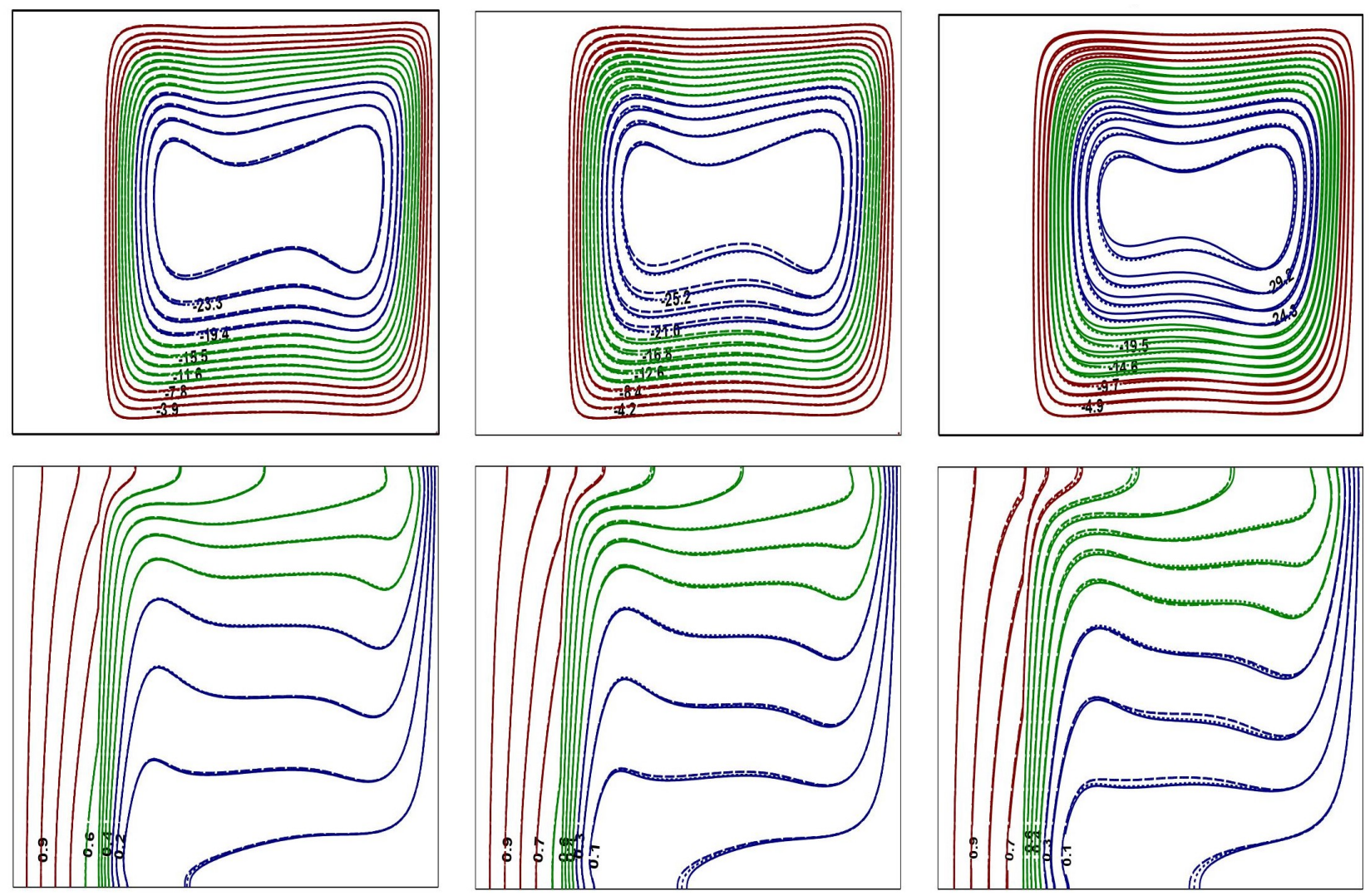

Figure 8. Impact of $\phi$ on flow and thermal contours at $R a=10^{6}, \varepsilon=0.2$ and $K r=5 . \phi=0.05$ (left), $\phi=0.1$ (center) and $\phi=0.2$ (right). (color online)

global $N u$ reveals an important prediction that the change in thermal resistance of the wall, due to variations of wall thickness, has profound influence on thermal dissipation rates along both solid cylinder and interface. The variation in thermal transport rate for different nanofluids could be vividly noticed along the interface. For all the values of $\phi$ and $\varepsilon$, it is noted that the role of $C u$-water in heat transfer enhancement is significant compared to other nanofluids.

The boundary conditions are chosen in the present analysis in such a way that the interior and exterior cylindrical boundaries are retained at different uniform temperatures, due to which the heat is transferred from inner surface towards outer cylinder. The thermal dissipation rate to nanofluid region greatly rely on the thermal variation along the solid-fluid interface. In addition, these thermal variations depend on various factors, such as the solid wall thickness, thermal conductivity ratio, Rayleigh number and nanoparticle concentrations. Thus, it is important to discuss the temperature changes alongside the interface in order to study the heat dissipation and flow characteristics in the annular region. Figure 14 shows the variation of solid-fluid interface temperature for various magnitudes of wall thickness at $R a=10^{6}, K r=2$ and $\phi=0.2$. A similar kind of linear variation in thermal profiles along the interface is observed for thinner $(\varepsilon=0.1)$ and thicker $(\varepsilon=0.5)$ cylinders. Also, the maximum temperature occurs near the upper portion of interface irrespective of wall thickness. This prediction might be from the fact that the clockwise rotating hot fluid rises upwards and hence the interface temperature increases along the height of the wall. Also, for all wall thickness, the temperature of $\mathrm{Al}_{2} \mathrm{O}_{3}$ nanofluid is higher compared to the nanofluid containing $\mathrm{TiO}_{2}$ and $C u$ nanoparticles.

The variation of interface temperature with thermal conductivity ratio for different nanofluids at $R a=10^{6}$, $\phi=0.2$ and $\varepsilon=0.2$ is presented in Fig. 15. As observed earlier, the temperature at interface increases along the axial direction with maximum temperature is being observed at top region for lower as well as higher conductivity ratios. For higher thermal conductivity ratio, the temperature along interface is found to be on larger scale since 
higher conductivity ratio indicates more wall heat conduction which brings a noticeable increase in the interface temperature. Further, it can be anticipated that the thickness of thermal boundary layer is more pronounced for higher $K r$ rather than lower $K r$. Figure 16(a) demonstrates the variation in solid-fluid interface temperature at $R a=10^{5}$, $K r=2$ and $\varepsilon=0.2$. For lower nanoparticle concentration $(\phi=0.05)$, the interface temperature is not altered by type of nanofluid considered. However, a noticeable change in temperature at the interface could be noticed for higher nanoparticle volume fraction $(\phi=0.2)$. An increasing trend in the interface temperature is observed for both the volume fractions. The impact of two Rayleigh numbers on the interface temperature is depicted in Fig. 16(b). The temperature at solid-fluid interface rises along the axial direction for all three nanofluids. In general, the interface temperature is found to be higher for lower Rayleigh number and for $\mathrm{Al}_{2} \mathrm{O}_{3}$-water nanofluid. Further, the interface temperature moderately increases with axial direction for lower $R a$, while a steep increase is found for higher $R a$. For $K r=2$, since wall conductivity is larger compared to nanofluid, more amount of heat is transferred to the nanofluid domain. Consequently, the temperature at interface declines.

\section{Conclusions}

Conjugate buoyant convective motion in an annular geometry with a solid inner cylinder is numerically analyzed. The annular region is filled with water based nanofluids having $\mathrm{Cu}, \mathrm{Al}_{2} \mathrm{O}_{3}$ and $\mathrm{TiO}_{2}$ nanoparticles. Based on the vast range of simulations, the obtained results can be summarized as follows:

1. The solid wall thickness is a significant factor which affects the flow variation and temperature distribution inside the annulus. An increment in the width of cylindrical wall leads to enhancement in the wall conductive resistance. As a result, thermal dissipation at the interface as well as the nanofluid motion declines. It can be concluded that, for all values of solid wall thickness, if $k_{w} \leq k_{n f}$ then $\overline{N u}_{w}>\overline{N u}_{i}$, however, for $k_{w}>k_{n f}$, the thermal dissipation at interface is found to be higher.

2. An increase in the solid wall to nanofluid thermal conductivity ratio $(K r)$ significantly increases the heat transport through the wall. Hence, convection is strengthened and the thermal transport through the interface is enhanced for higher values of $K r$. For given value of $\phi$ and $\varepsilon$, depending on the thermal conductivities of wall and nanofluid, the global Nusselt number is altered. If thermal conductivity of nanofluid is higher compared to solid wall, then the overall heat transport rate at the wall is higher compared to the interface $\left(\overline{N u}_{w}>\overline{N u}_{i}\right)$. The reverse trend has been noticed for the higher nanofluid thermal conductivity.

3. The addition of nano-sized particles to base fluid enhances the thermal dissipation at the solid-fluid interface and strengthens the nanofluid movement. The total heat transport rate for all three different nanofluids enhances with an increase in nanoparticle density. For any value of nanoparticle concentration, $\overline{N u}_{w}<\overline{N u}_{i}$ for fixed $K r$ and $\varepsilon$.

4. The type of nanoparticle influences the flow behavior and thermal transport rate in a great manner. For given values of $\mathrm{Ra}, \varepsilon, \mathrm{Kr}$ and $\phi$, the thermal transport rate is significantly higher for $\mathrm{Cu}-\mathrm{H}_{2} \mathrm{O}$ nanofluid compared to other two nanofluid considered in the present investigation. This prediction is due to the fact that $\mathrm{Cu}$ is having comparatively higher thermal conductivity.

$$
\overline{\mathrm{Nu}}(\mathrm{Cu})>\overline{\mathrm{Nu}}\left(\mathrm{Al}_{2} \mathrm{O}_{3}\right)>\overline{\mathrm{Nu}}\left(\mathrm{TiO}_{2}\right)
$$

5. To study the thermal dissipation and flow behavior throughout the domain, the temperature variations along the interface is analyzed. For all values of $R a, \phi, \varepsilon$ and $K r$, the upper portion of interface exhibits higher temperature.

6. The choice of parameters play a key role in providing enhanced thermal performance. For minimal wall thickness, the choice of higher values of $R a, K r$ and $\phi$ enhances the heat transfer through the interface for all the three nanofluids considered in the current analysis. 


\section{References}

1. de Vahl Davis, G. \& Thomas, R. W. Natural convection between concentric vertical cylinders. Phys. Fluids 12, 198-207 (1969).

2. Kumar, R. \& Kalam, M. A. Laminar thermal convection between vertical coaxial isothermal cylinders. Int. J. Heat Mass Transfer 34(2), 513-524 (1991).

3. Prasad, V. \& Kulacki, F. A. Free convection heat transfer in a liquid-filled annulus. ASME J. Heat Transfer 107, 596-602 (1985).

4. Sankar, M., Venkatachalappa, M. \& Shivakumara, I. S. Effect of magnetic field on natural convection in a vertical cylindrical annulus. Int. J. Eng. Sci. 44, 1556-1570 (2006).

5. Kakarantzas, S. C., Sarris, I. E. \& Vlachos, N. S. Natural convection of liquid metal in a vertical annulus with lateral and volumetric heating in the presence of a horizontal magnetic field. Int. J. Heat Mass Transfer 54, 3347-3356 (2011).

6. Afrand, M., Toghraie, D., Karimipour, A. \& Wongwises, S. A numerical study of natural convection in a vertical annulus filled with gallium in the presence of magnetic field. J. Magn. Magn. Mater. 430, 22-28 (2017).

7. Altmeyer, S. \& Do, Y. Effects of an imposed axial flow on a ferrofluidic Taylor-Couette flow. Sci. Rep. 9, 15438 (2019).

8. Sankar, M. \& Do, Y. Numerical simulation of free convection heat transfer in a vertical annular cavity with discrete heating. Int. Commun. Heat Mass Transfer 37, 600-606 (2010).

9. Sankar, M., Park, Y., Lopez, J. M. \& Do, Y. Numerical study of natural convection in a vertical porous annulus with discrete heating. Int. J. Heat Mass Transfer 54, 1493-1505 (2011).

10. Wang, S., Faghri, A. \& Bergman, T. L. Transient natural convection in vertical annuli: Numerical modeling and heat transfer correlation. Numer. Heat Transf. A 61(11), 823-836 (2012).

11. Mebarek-Oudina, F. Numerical modeling of the hydrodynamic stability in vertical annulus with heat source of different lengths. Int. J. Eng. Sci. Tech. 20, 1324-1333 (2017).

12. Husain, S. \& Siddiqui, M. A. Experimental and numerical analysis of transient natural convection of water in a high aspect ratio narrow vertical annulus. Prog. Nucl. Energy 106, 1-10 (2018).

13. Choi, S. U. S. \& Eastman, J. A. Enhancing thermal conductivity of fluids with nanoparticles. ASME Int. Mech. Eng. Congr. Expo. (1995).

14. Putra, N., Roetzel, W. \& Das, S. K. Natural convection of nano-fluids. Heat Mass Transfer 39, 775-784 (2003).

15. Abouali, O. \& Falahatpisheh, A. Numerical investigation of natural convection of $\mathrm{Al}_{2} \mathrm{O}_{3}$ nanofluid in vertical annuli. Heat Mass Transfer 46, 15-23 (2009).

16. Cadena-de la Peña, N. L., Rivera-Solorio, C. I., Payán-Rodríguez, L. A., García-Cuéllar, A. J. \& López-Salinas, J. L. Experimental analysis of natural convection in vertical annuli filled with AlN and $\mathrm{TiO}_{2} / \mathrm{mineral}$ oil-based nanofluids. Int. J. Therm. Sci. 111, 138-145 (2017).

17. Mebarek-Oudina, F. Convective heat transfer of titania nanofluids of different base fluids in cylindrical annulus with discrete heat source. Heat Transfer-Asian Res. 48(1), 135-147 (2019).

18. Mebarek-Oudina, F., Keerthi Reddy, N. \& Sankar, M. Heat source location effects on buoyant convection of nanofluids in an annulus. (2021) B. Rushi Kumar et al. (eds.), Advances in Fluid Dynamics, Lecture Notes in Mechanical Engineering, https://doi.org/10.1007/978-981-15-4308-1-70.

19. Keerthi Reddy, N. \& Sankar, M. Buoyant convective transport of nanofluids in a non-uniformly heated annulus. J. Phys.: Conf. Ser. 1597, 012055 (2020). 
20. Abu-Nada, E., Masoud, Z. \& Hijazi, A. Natural convection heat transfer enhancement in horizontal concentric annuli using nanofluids. Int. Commun. Heat Mass Transfer 35, 657-665 (2008).

21. Sultan, K. F. Numerical solution of heat transfer and flow of nanofluids in annulus with fins attached on the inner cylinder. J. Babylon University/Eng. Sci. 23(2), 465-484 (2015).

22. Khanafer, K., Vafai, K. \& Lightstone, M. Buoyancy-driven heat transfer enhancement in a two-dimensional enclosure utilizing nanofluids. Int. J. Heat Mass Transfer 46, 3639-3653 (2003).

23. Jou, R. \& Tzeng, S. Numerical research of nature convective heat transfer enhancement filled with nanofluids in rectangular enclosures. Int. Commun. Heat Mass Transfer 33, 727-736 (2006).

24. Santra, A. K., Sen, S. \& Chakraborty, N. Study of heat transfer augmentation in a differentially heated square cavity using copper-water nanofluid. Int. J. Therm. Sci. 47, 1113-1122 (2008).

25. Abu-Nada, E., Masoud, Z., Oztop, H. F. \& Campo, A. Effect of nanofluid variable properties on natural convection in enclosures. Int. J. Therm. Sci. 49, 479-491 (2010).

26. Ho, C. J., Chen, M. W. \& Li, Z. W. Numerical simulation of natural convection of nanofluid in a square enclosure: Effects due to uncertainties of viscosity and thermal conductivity. Int. J. Heat Mass Transfer 51, 4506-4516 (2008).

27. Oztop, H. F. \& Abu-Nada, E. Numerical study of natural convection in partially heated rectangular enclosures filled with nanofluids. Int. J. Heat Fluid Flow 29, 1326-1336 (2008).

28. Basak, T. \& Chamkha, A. J. Heatline analysis on natural convection for nanofluids confined within square cavities with various thermal boundary conditions. Int. J. Heat Mass Transfer 55, 5526-5543 (2012).

29. Alsabery, A. I., Chamkha, A. J. \& Saleh, H. Natural convection flow of a nanofluid in an inclined square enclosure partially filled with a porous medium. Sci. Rep. 7, 2357 (2017).

30. Roy, N. C. Natural convection of nanofluids in a square enclosure with different shapes of inner geometry. Phys. Fluids 30, 113605 (2018).

31. Sharaf, O. Z., Al-Khateeb, A. N., Kyritsis, D. C. \& Abu-Nada, E. Numerical investigation of nanofluid particle migration and convective heat transfer in microchannels using an Eulerian-Lagrangian approach. J. Fluid Mech. 878, 62-97 (2019).

32. Bendaraa, A., Charafi, M. M. \& Hasnaoui, A. Numerical study of natural convection in a differentially heated square cavity filled with nanofluid in the presence of fins attached to walls in different locations. Phys. Fluids 31, 052003 (2019).

33. Abu-Nada, E. \& Oztop, H. F. Numerical analysis of $\mathrm{Al}_{2} \mathrm{O}_{3} /$ water nanofluids natural nonvection in a wavy walled cavity. Numer. Heat Transf. A 59(5), 403-419 (2011).

34. Ghalambaz, M., Sheremet, M. A. \& Pop, I. Free convection in a parallelogrammic porous cavity filled with a nanofluid using Tiwari and Das' nanofluid model. PLoS One 10(5), e0126486 (2015).

35. Vanaki, Sh. M., Ganesan, P. \& Mohammed, H. A. Numerical study of convective heat transfer of nanofluids: A review. Renew. Sust. Energy Rev. 54, 1212-1239 (2016).

36. Guo, Z. A review on heat transfer enhancement with nanofluids. J. Enhanc. Heat Transf. 27(1), 1-70 (2020).

37. Kaminski, D. A. \& Prakash, C. Conjugate natural convection in a square enclosure: effect of conduction in one of the vertical walls. Int. J. Heat Mass Transfer 29(12), 1979-1988 (1986).

38. Ben-Nakhi, A. \& Chamkha, A. J. Conjugate natural convection in a square enclosure with inclined thin fin of arbitrary length. Int. J. Therm. Sci. 46(5), 467-478 (2007).

39. Belazizia, A., Benissaad, S. \& Abboudi, S. Effect of magnetic field and wall conductivity on conjugate natural convection in a square enclosure. Arab J. Sci. Eng. 39, 4977-4989 (2014). 
40. Saeid, N. H. Conjugate natural convection in a vertical porous layer sandwiched by finite thickness walls. Int. Commun. Heat Mass Transfer 34(2), 210-216 (2007).

41. Ghalambaz, M., Tahmasebi, A., Chamkha, A. J. \& Wen, D. Conjugate local thermal non-equilibrium heat transfer in a cavity filled with a porous medium: Analysis of the element location. Int. J. Heat Mass Transfer 138, 941-960 (2019).

42. Aminossadati, S. M. \& Ghasemi, B. Conjugate natural convection in an inclined nanofluid-filled enclosure. Int. J. Numer. Meth. Heat Fluid Flow 22(4), 403-423 (2012).

43. Alsabery, A. I., Sheremet, M. A. \& Chamkha, A. J. MHD convective heat transfer in a discretely heated square cavity with conductive inner block using two-phase nanofluid model. Sci. Rep. 8, 7410 (2018).

44. Zadeh, S. M. H., Sabour, M., Sazgara, S. \& Ghalambaz, M. Free convection flow and heat transfer of nanofluids in a cavity with conjugate solid triangular blocks: Employing Buongiorno's mathematical model. Physica A 538, 122826 (2020).

45. Sheremet, M. A. \& Pop, I. Conjugate natural convection in a square porous cavity filled by a nanofluid using Buongiorno's mathematical model. Int. J. Heat Mass Transfer 79, (2014) 137-145.

46. Alsabery, A.I., Chamkha, A.J., Saleh, H. \& Hashim, I. Heatline visualization of conjugate natural convection in a square cavity filled with nanofluid with sinusoidal temperature variations on both horizontal walls. Int. J. Heat Mass Transfer 100, 835-850 (2016).

47. Zahan, I. \& Alim, M. A. Effect of conjugate heat transfer on flow of nanofluid in a rectangular enclosure. Int. J. Heat Technol. 36(2), 397-405 (2018).

48. Bondarenko, D. S., Sheremet, M. A., Oztop, H. F. \& Ali, M. E. Natural convection of $\mathrm{Al}_{2} \mathrm{O}_{3} / \mathrm{H}_{2} \mathrm{O}$ nanofluid in a cavity with a heat-generating element: Heatline visualization. Int. J. Heat Mass Transfer 130, 564-574 (2019).

49. Ghalambaz, M., Doostani, A., Izadpanahi, E. Conjugate natural convection flow of Ag-MgO/water hybrid nanofluid in a square cavity. J. Therm. Anal. Calorim. 139, 2321-2336 (2019).

50. Venkata Reddy, P. \& Narasimham, G. S. V. L. Natural convection in a vertical annulus driven by a central heat generating rod. Int. J. Heat Mass Transfer 51, 5024-5032 (2008).

51. Badruddin, I. A., Ahmed N. J. S. \& Al-Rashed, A. A. A. A. Conjugate heat transfer in an annulus with porous medium fixed between solids. Transp. Porous Med. 109, 589-608 (2015).

52. John, B., Senthilkumar, P. \& Sadasivan, S. Applied and theoretical aspects of conjugate heat transfer analysis: A review. Arch. Computat. Meth. Eng. 26, 475-489 (2019).

\section{Acknowledgement}

The authors M.S. and N.K.R. acknowledge the Presidency University and VGST for the support and encouragement. Y.D. was supported by the National Research Foundation of Korea (NRF) grant funded by the Korea government(MSIP) (No. NRF-2019R1A2B5B01070579).

\section{Author contributions}

M.S. and Y.D. designed the research problem and developed code. N.K.R. performed numerical simulations and plotted the graphs. M.S., N.K.R. and Y.D. analyzed the results and wrote the paper.

\section{Conflict of interest}

The authors declare that they have no conflict of interest. 


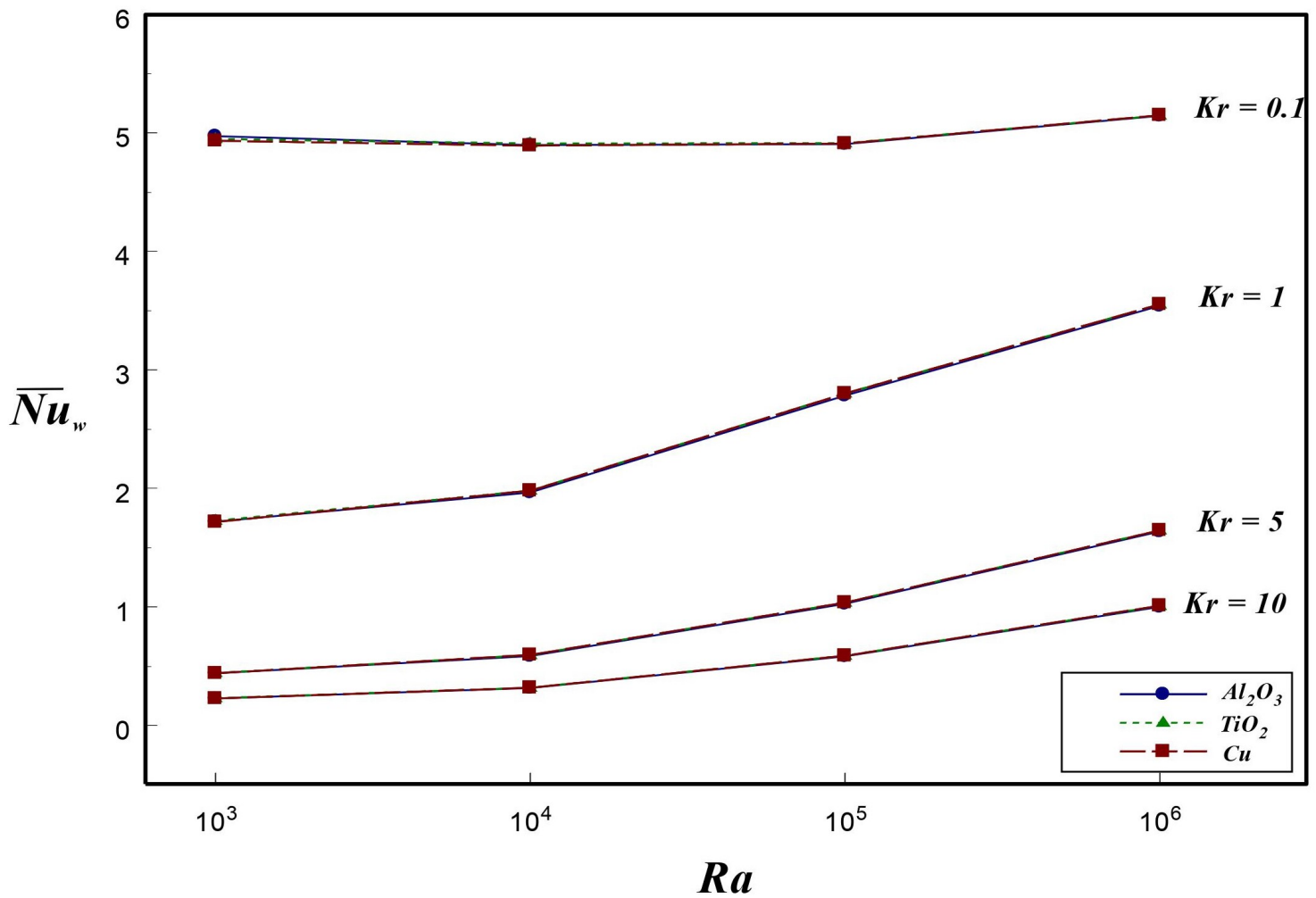

(a)

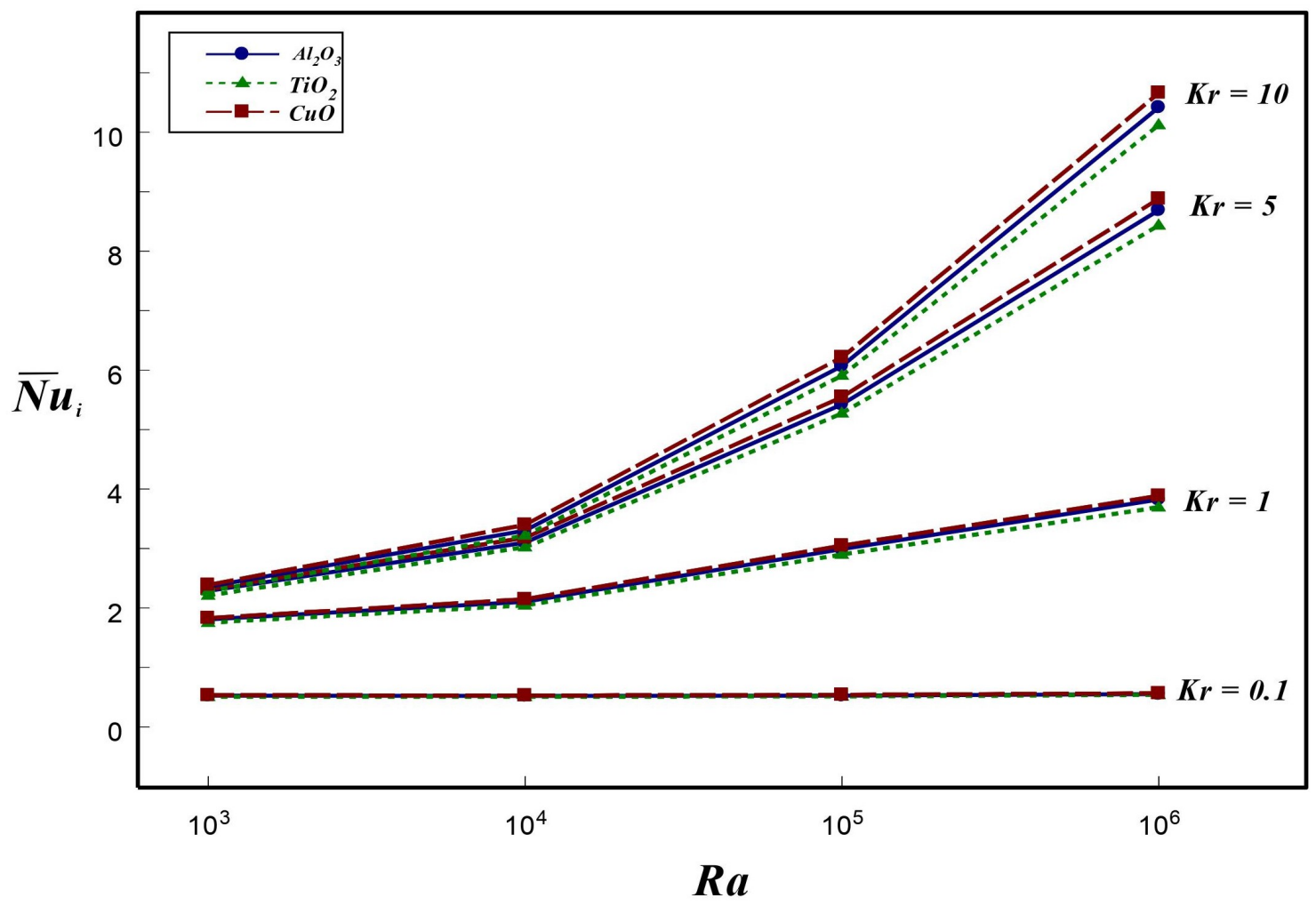

(b)

Figure 9. Effect of $R a$ and $K r$ on $\overline{N u}$ at the (a) wall and (b) interface for $\varepsilon=0.2$ and $\phi=0.1$. (color online) 


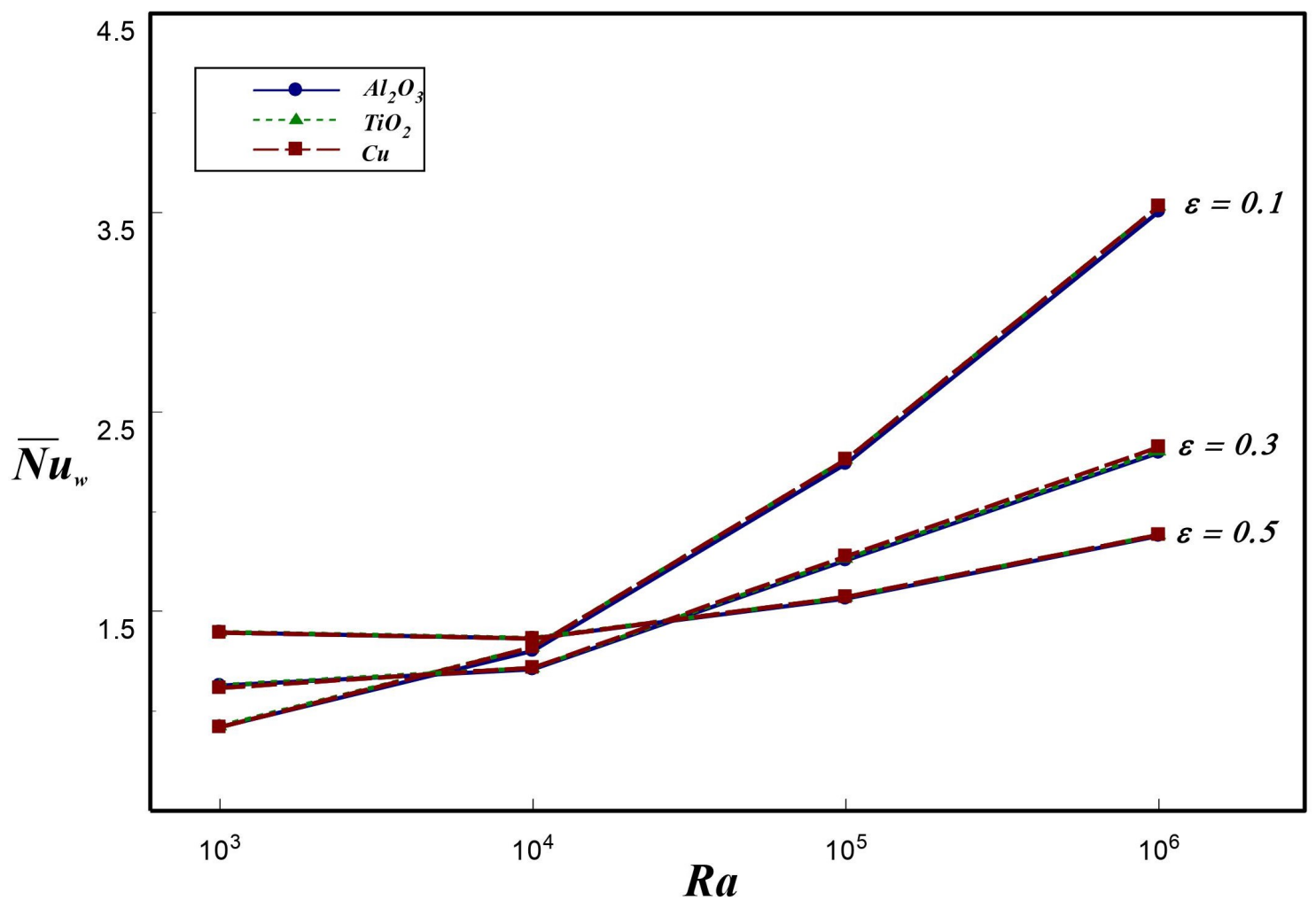

(a)

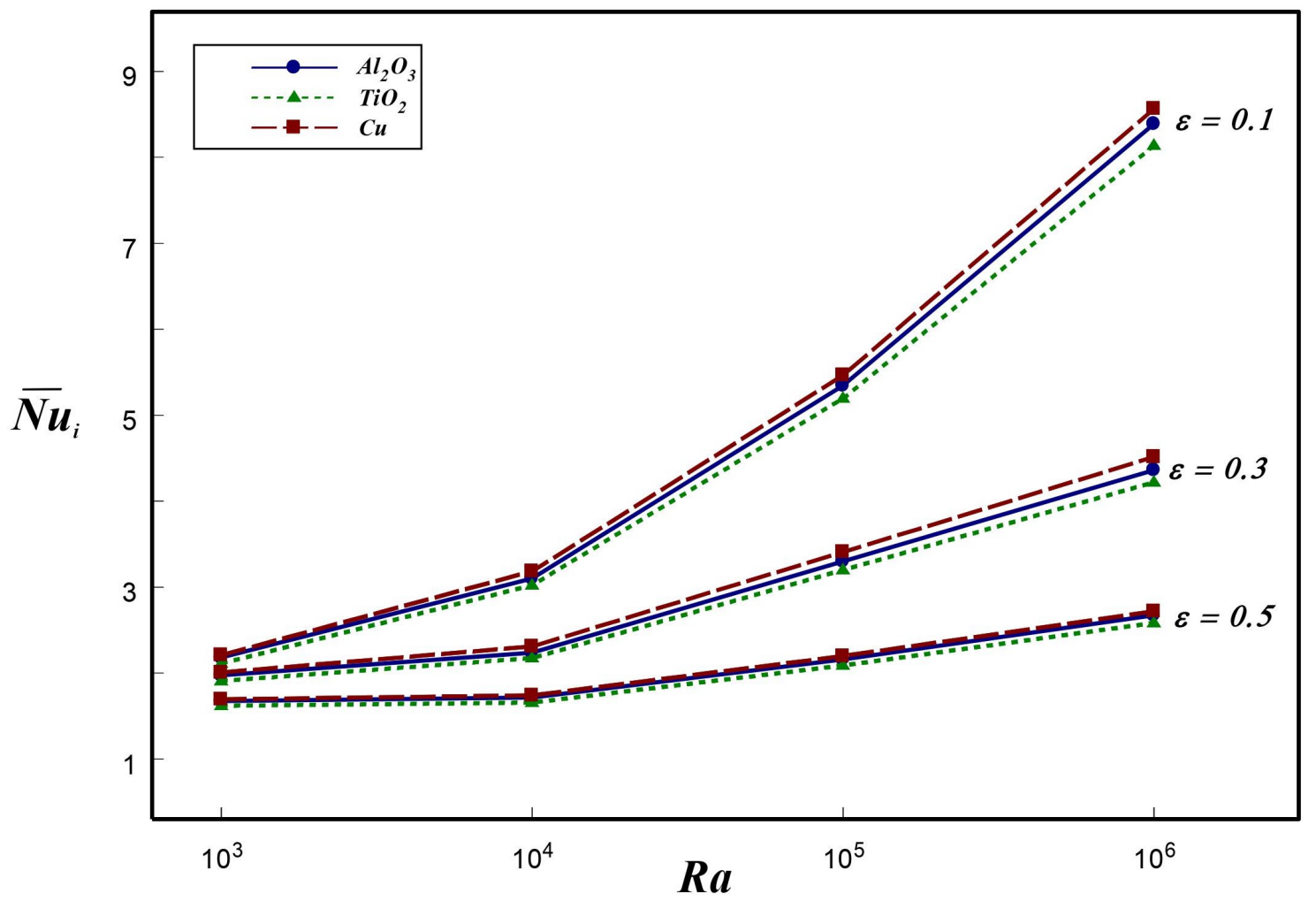

(b)

Figure 10. Effect of $R a$ and $\varepsilon$ on $\overline{N u}$ at the (a) wall and (b) interface for $K r=2$ and $\phi=0.1$. (color online) 


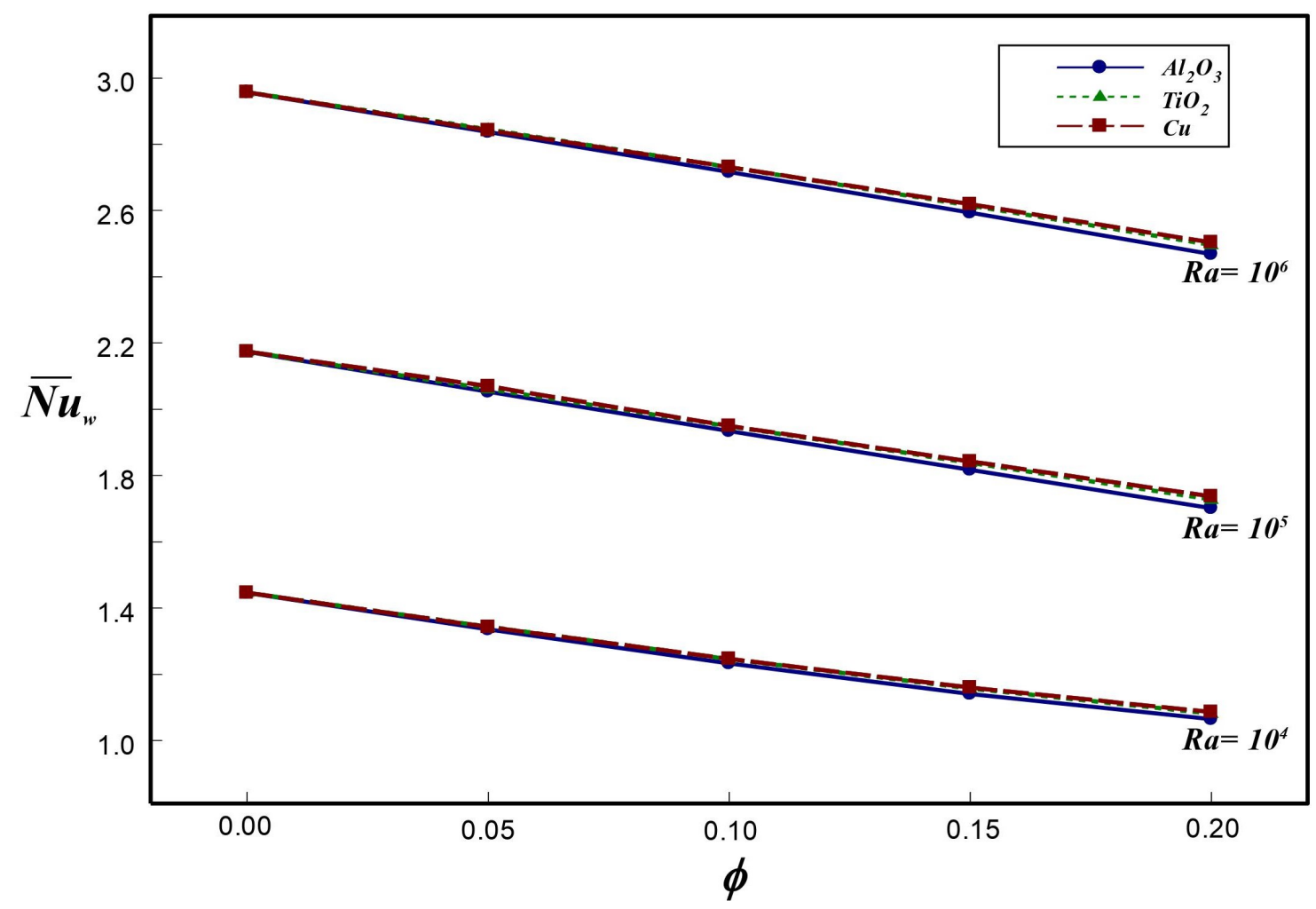

(a)

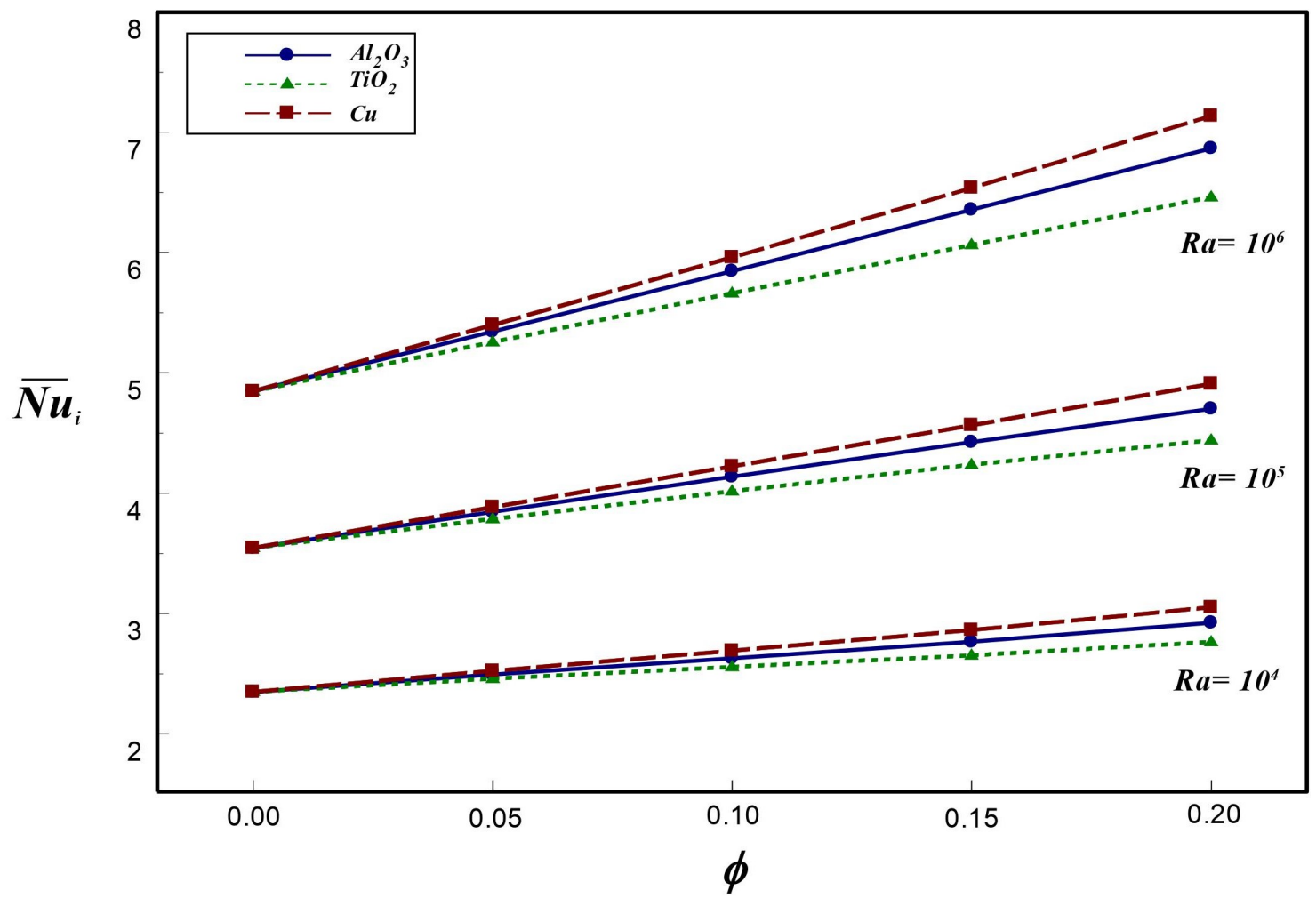

(b)

Figure 11. Effect of $R a$ and $\phi$ on $\overline{N u}$ at the (a) wall and (b) interface for $K r=2$ and $\varepsilon=0.2$. (color online) 


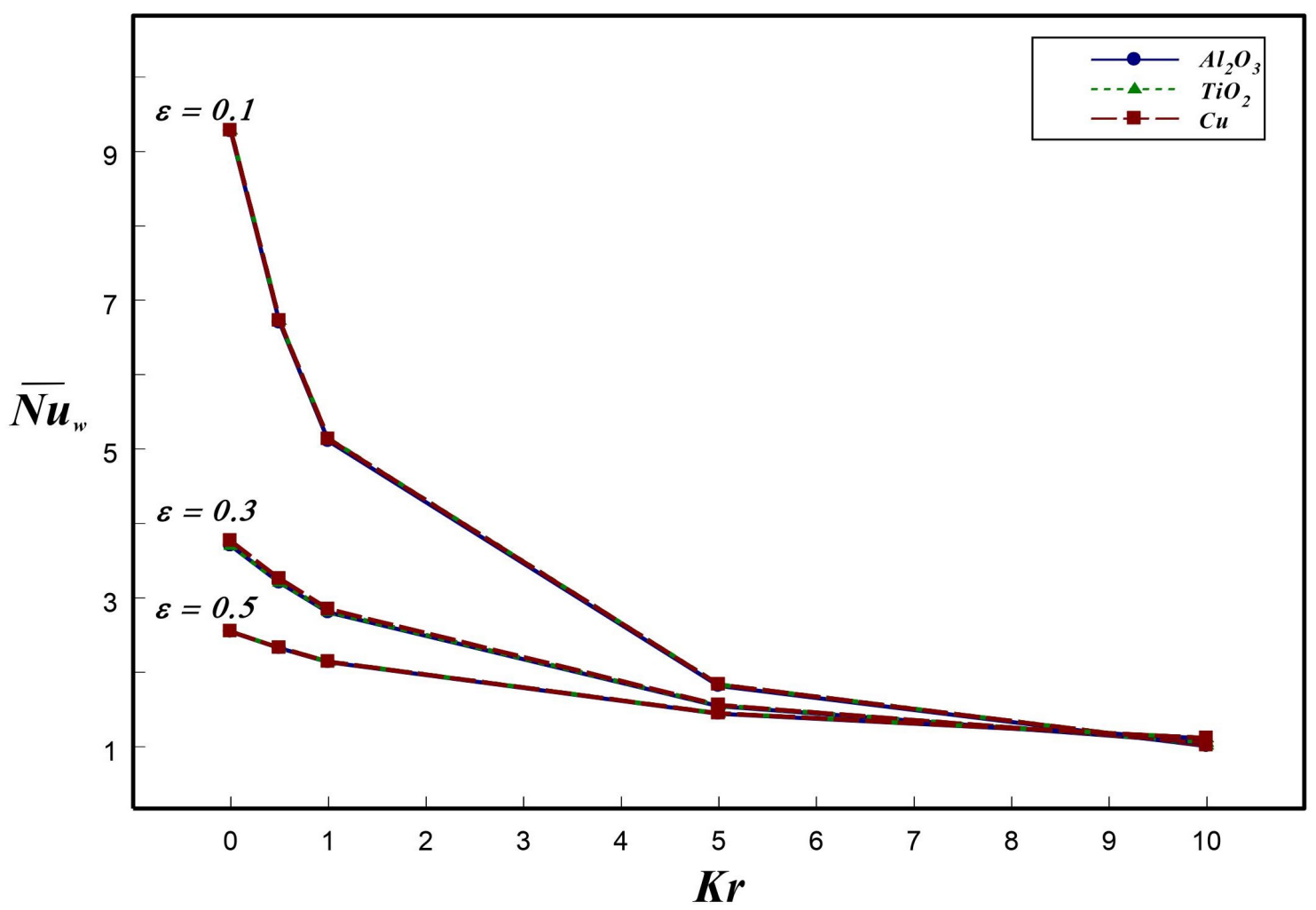

(a)

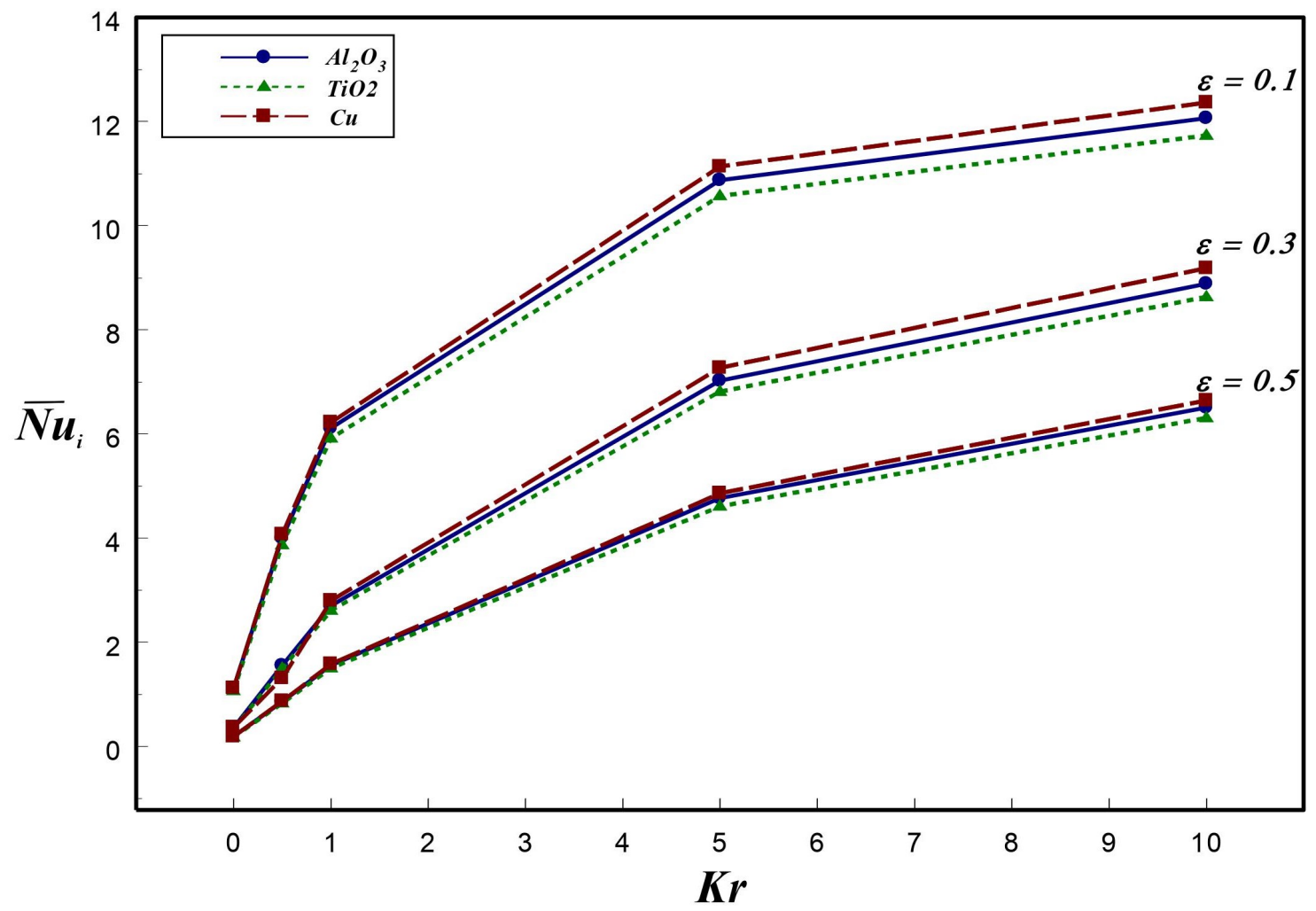

(b)

Figure 12. Effect of $K r$ and $\varepsilon$ on $\overline{N u}$ at the (a) wall and (b) interface for $R a=10^{6}$ and $\phi=0.1$. (color online) 


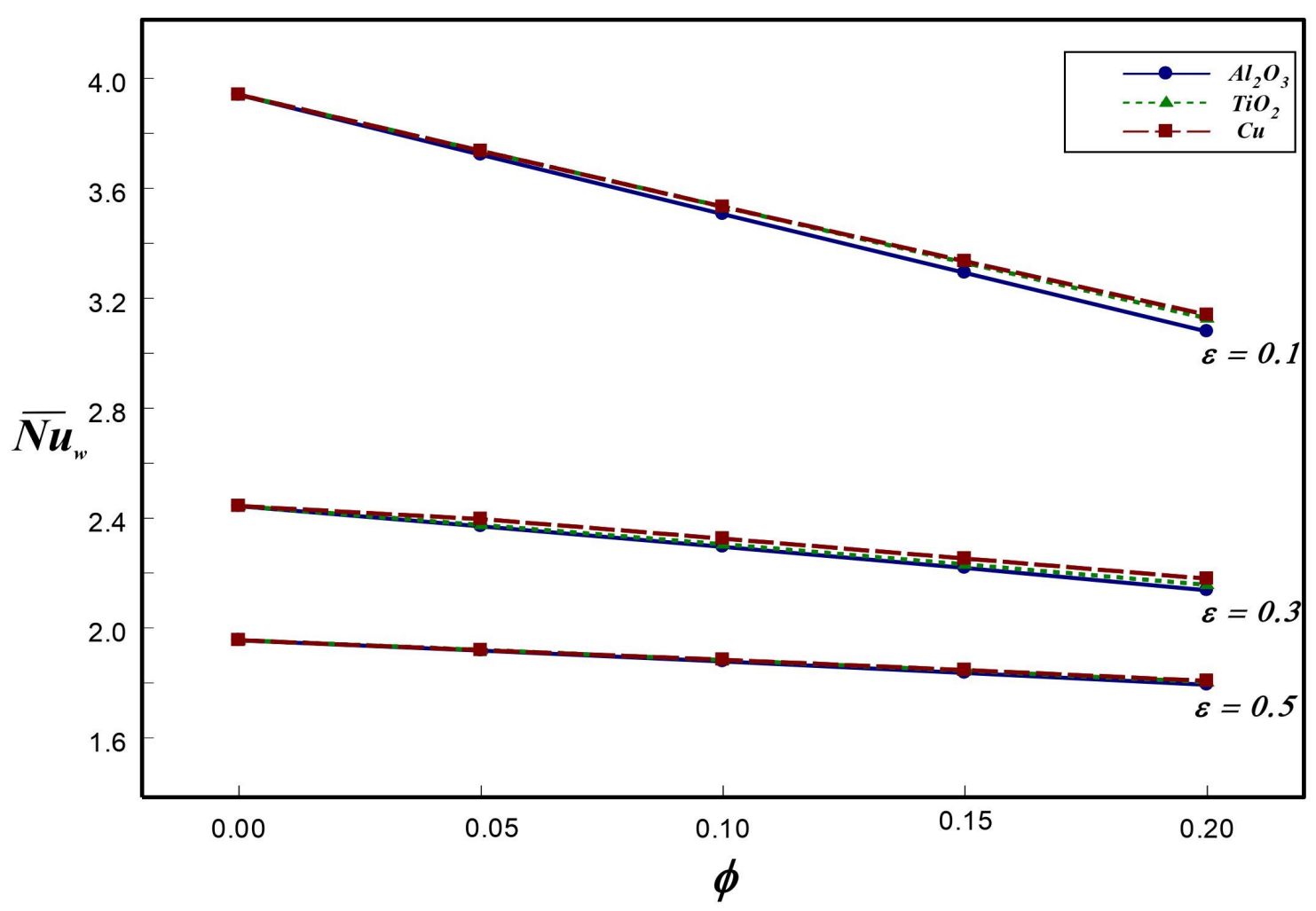

(a)

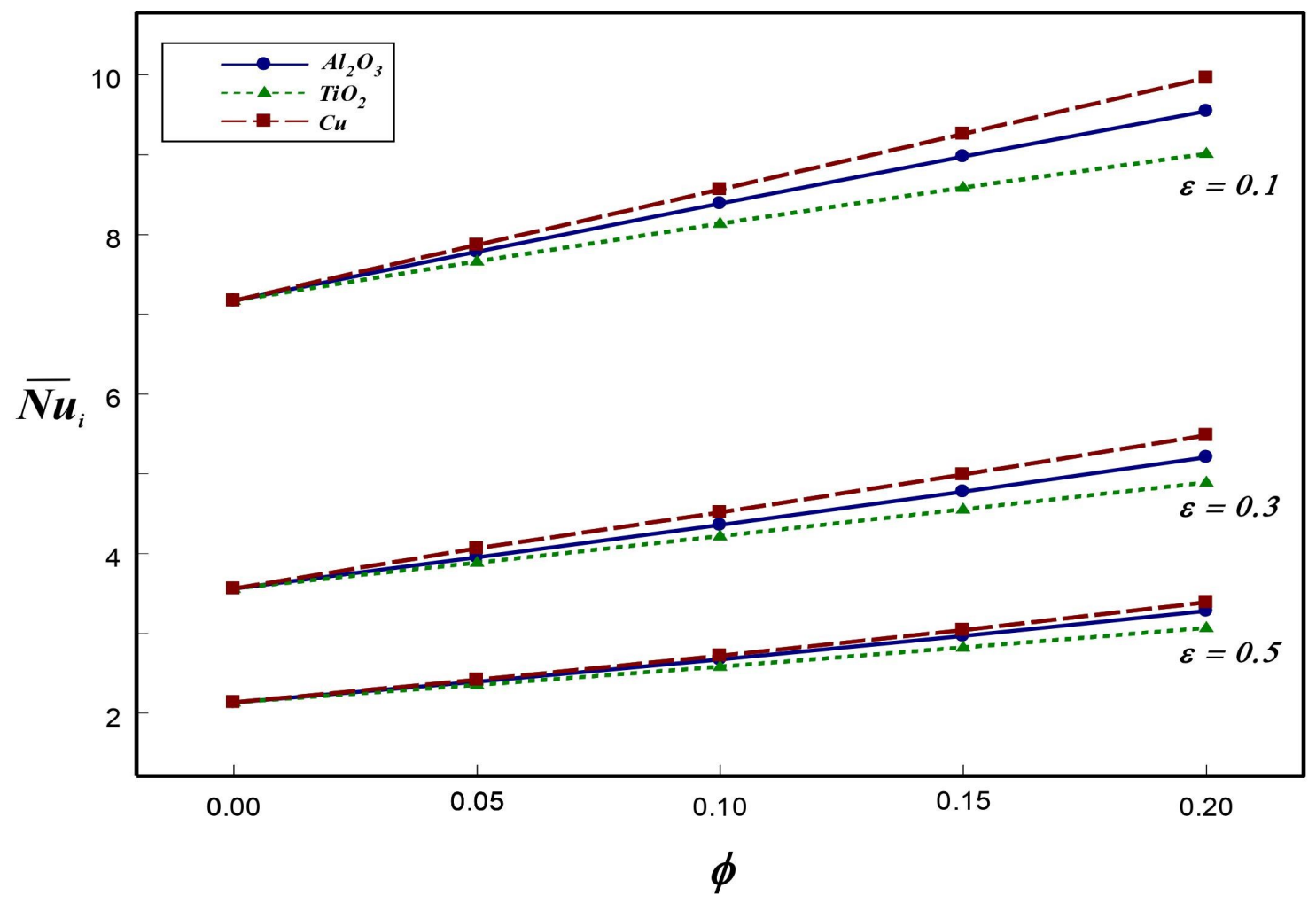

(b)

Figure 13. Effect of $\phi$ and $\varepsilon$ on $\overline{N u}$ at the (a) wall and (b) interface for $R a=10^{6}$ and $K r=2$. (color online) 


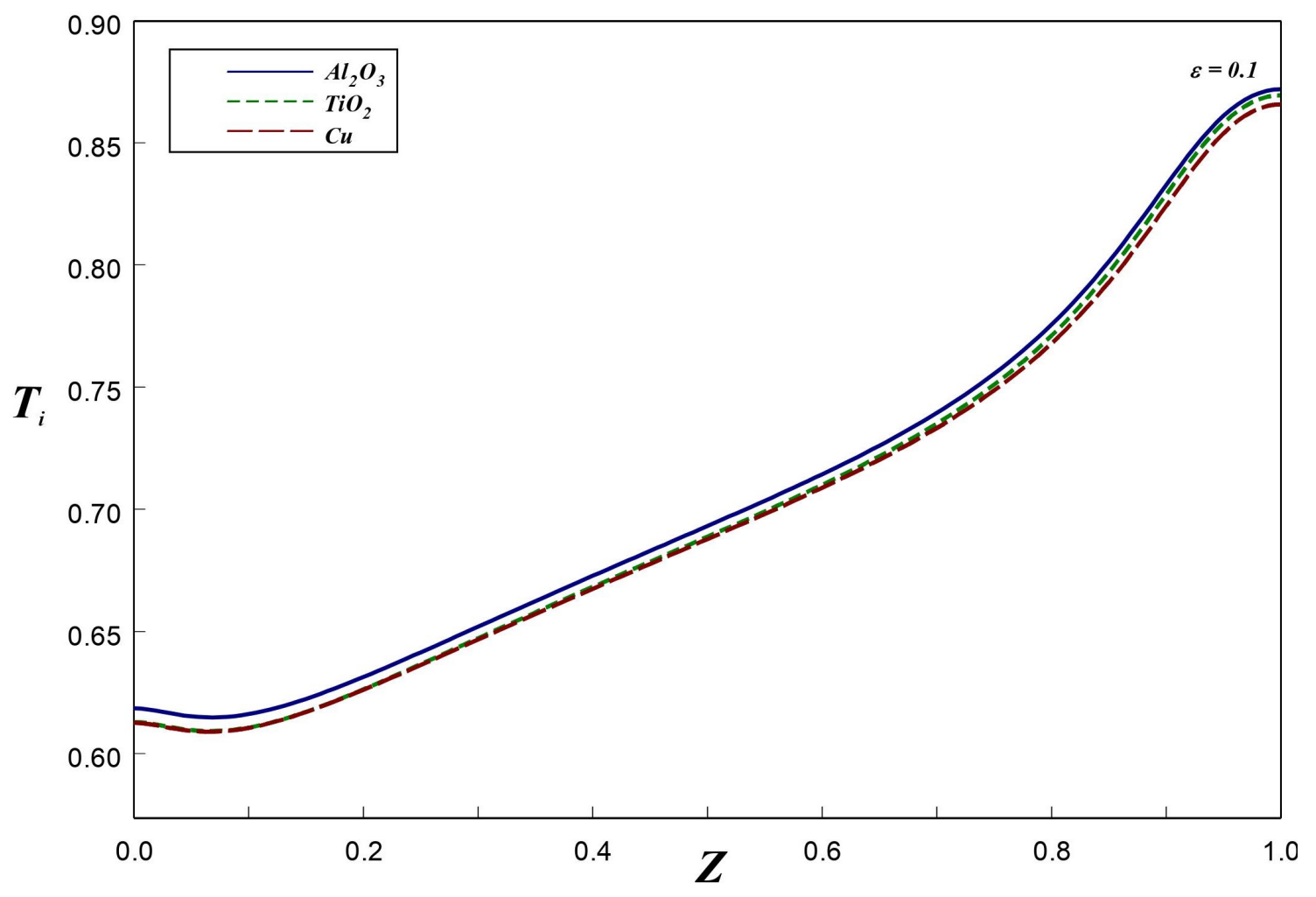

(a)

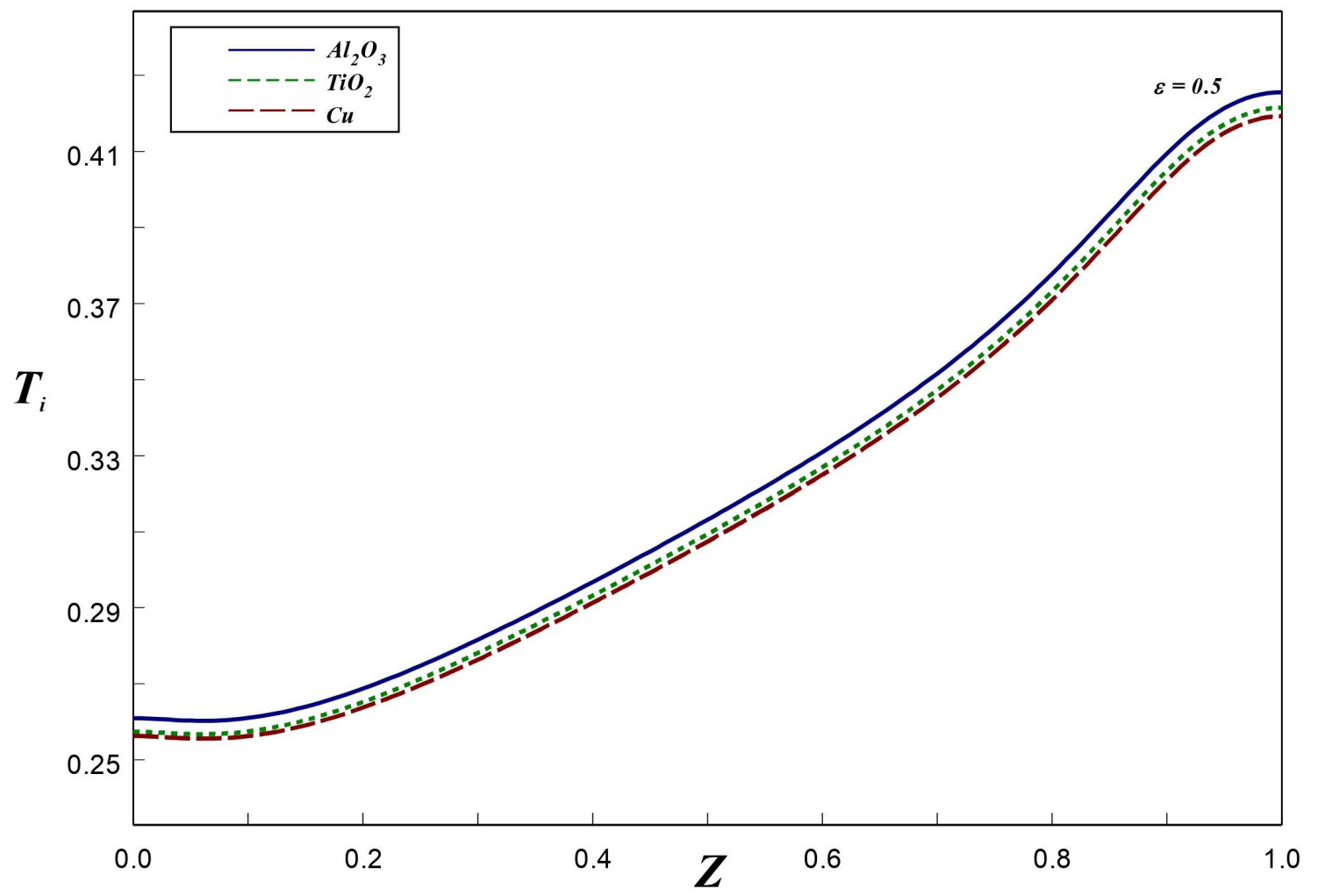

(b)

Figure 14. Axial variation of interface temperature with different wall thickness (a) $\varepsilon=0.1$ and (b) $\varepsilon=0.5$. (color online) 


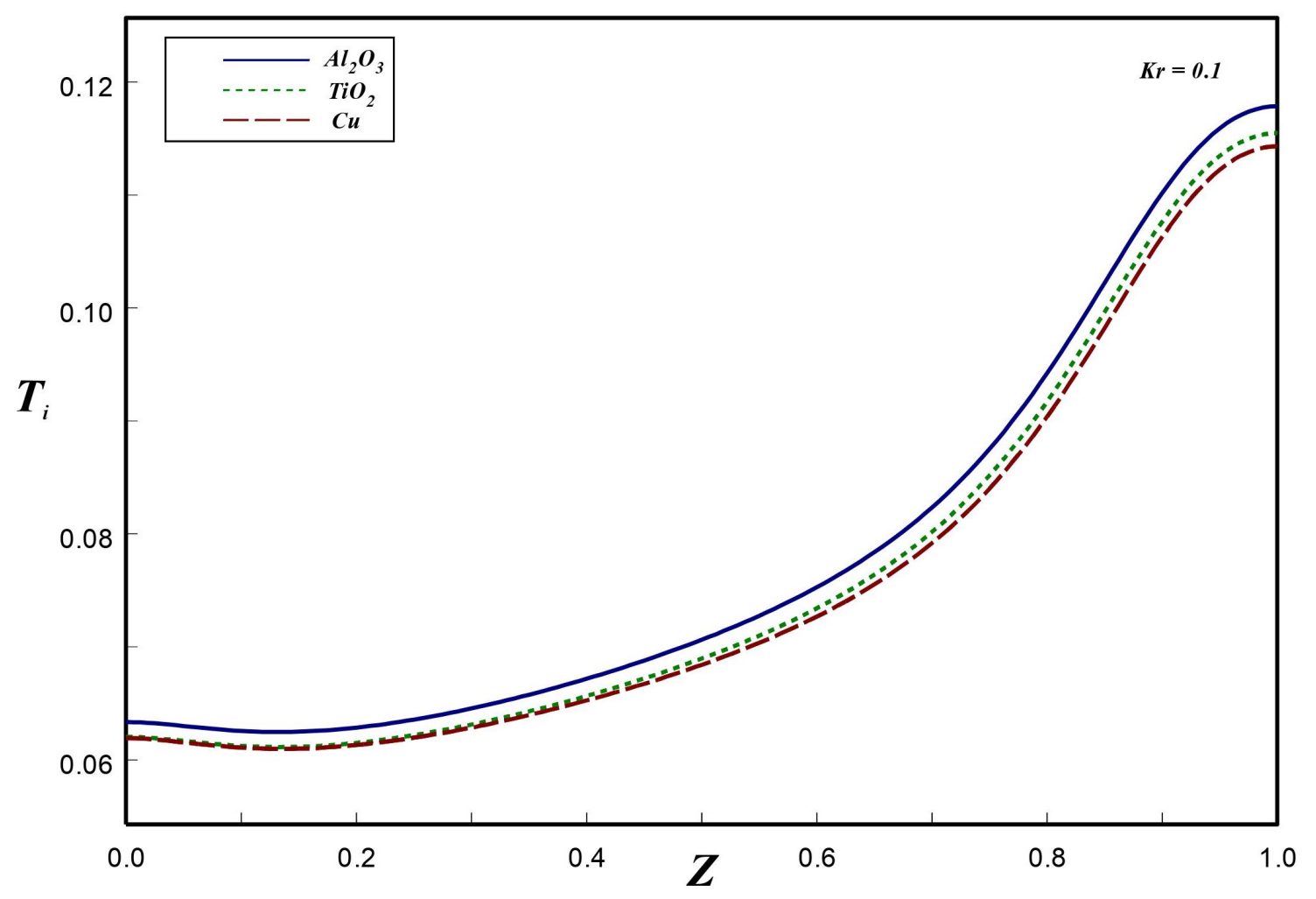

(a)

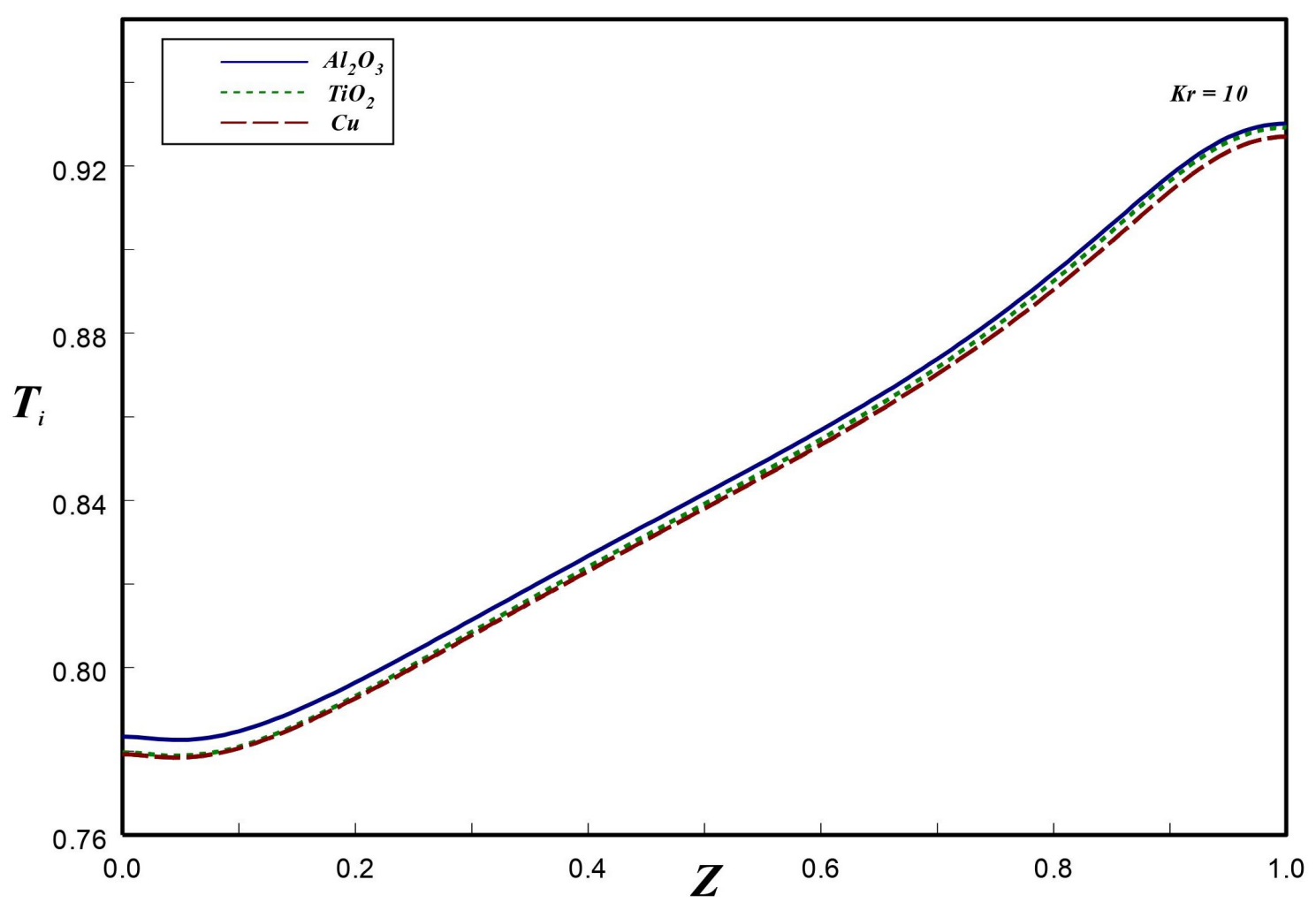

(b)

Figure 15. Axial variation of interface temperature with different thermal conductivity ratio (a) $K r=0.1$ and (b) $K r=10$. (color online) 


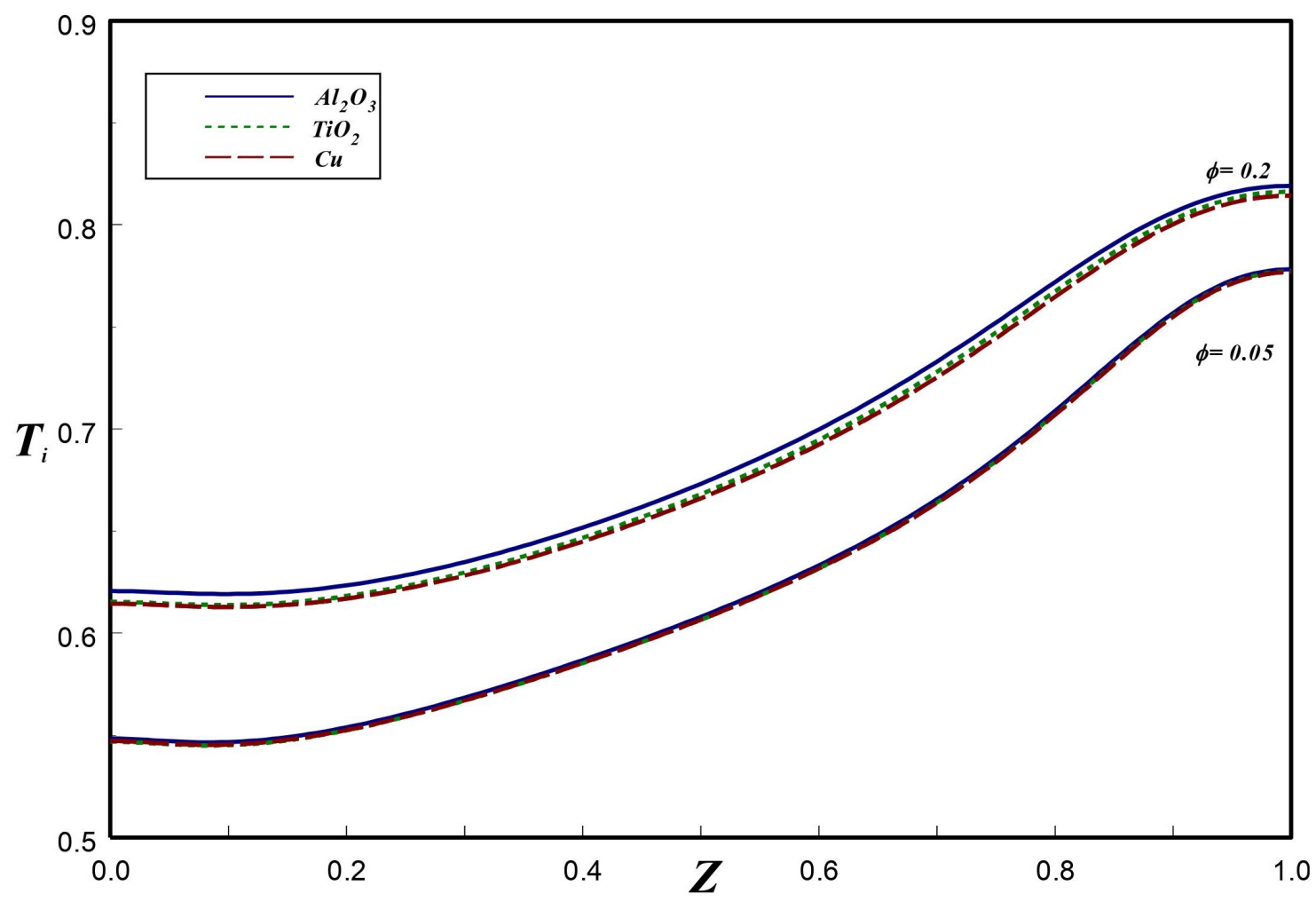

(a)

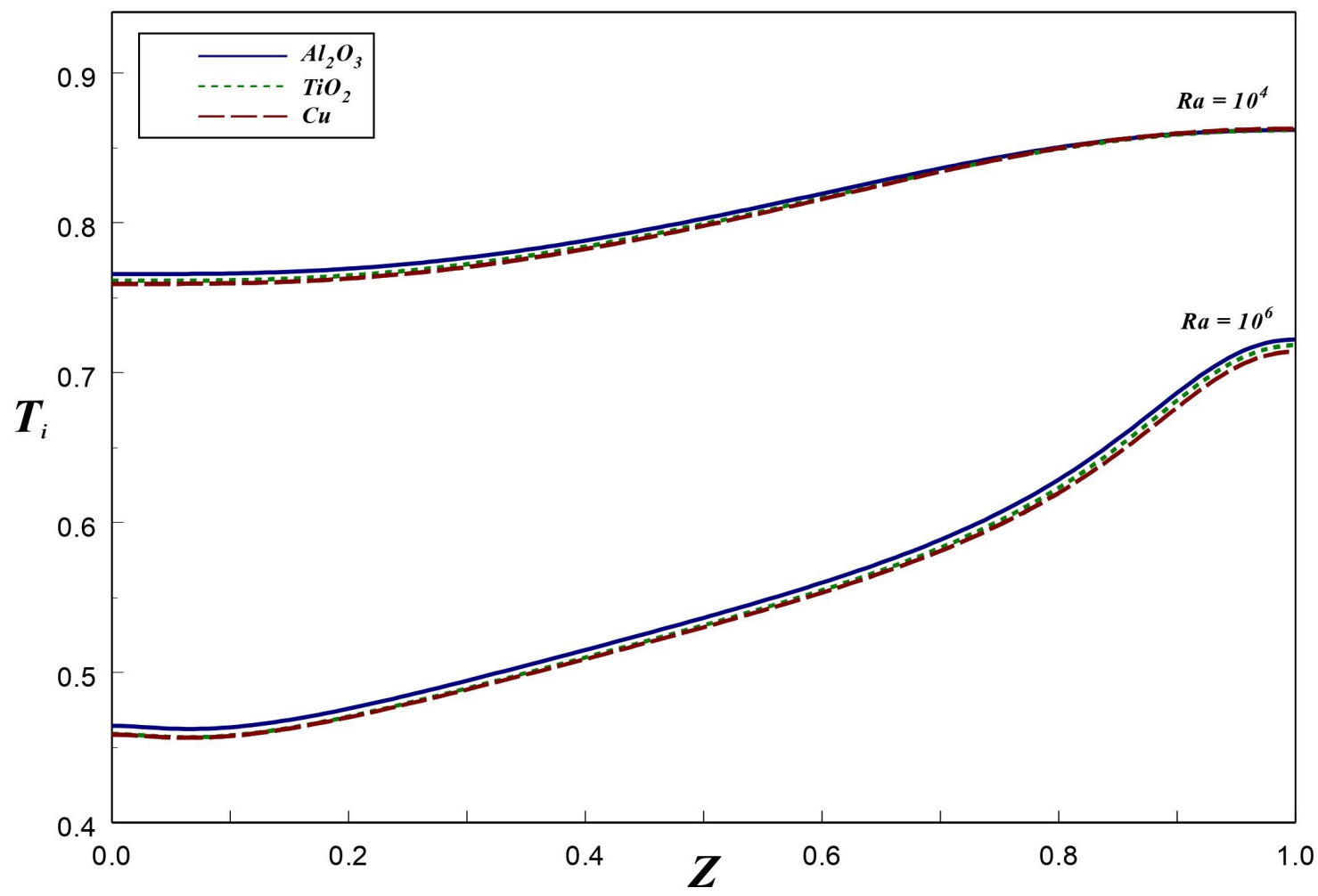

(b)

Figure 16. Axial variation of interface temperature with different (a) nanoparticle volume fraction $(\phi)$ and (b) Rayleigh number $(R a)$. (color online) 
Figures

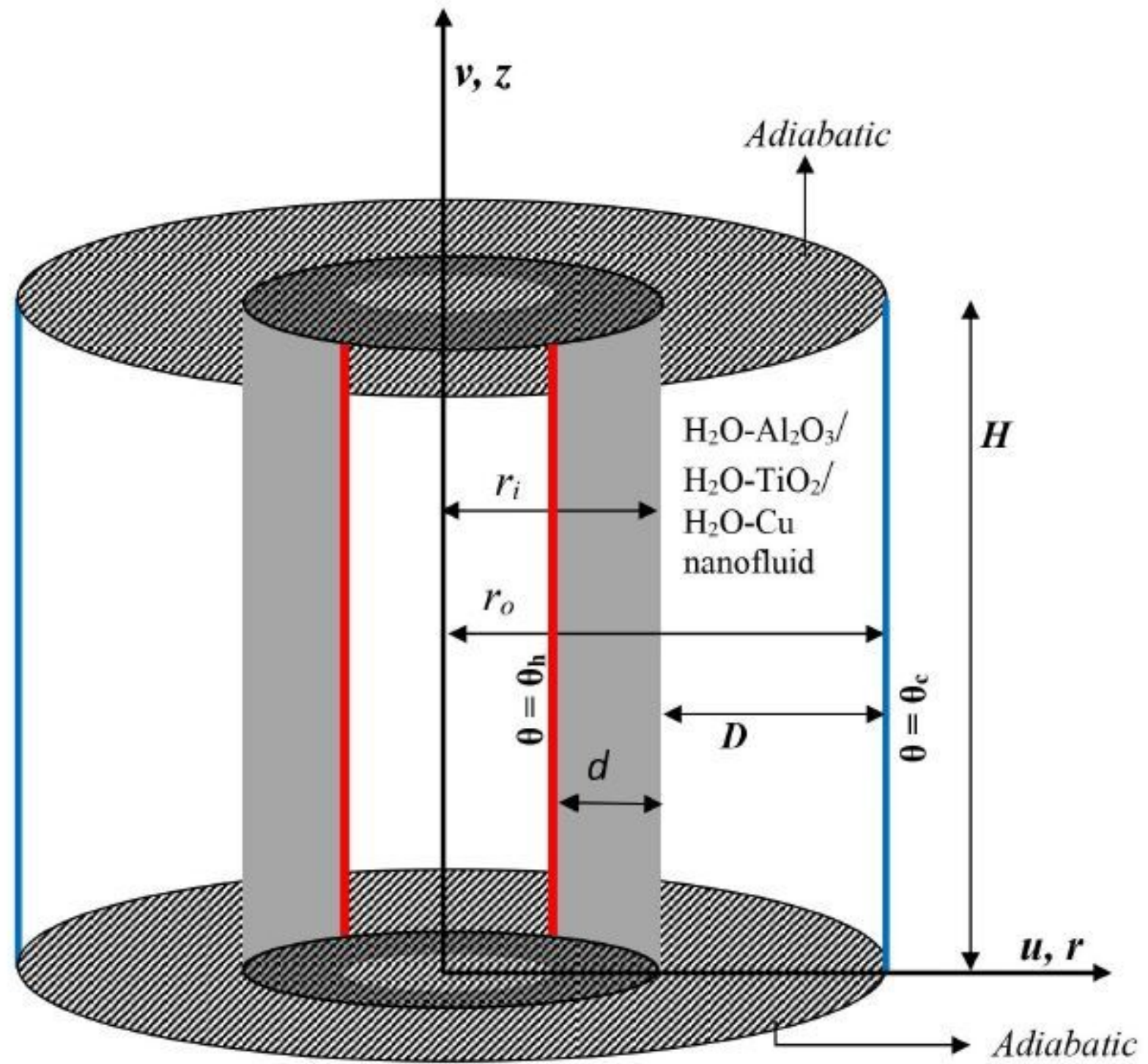

Figure 1

The annular geometry, Coordinate system and thermal conditions 

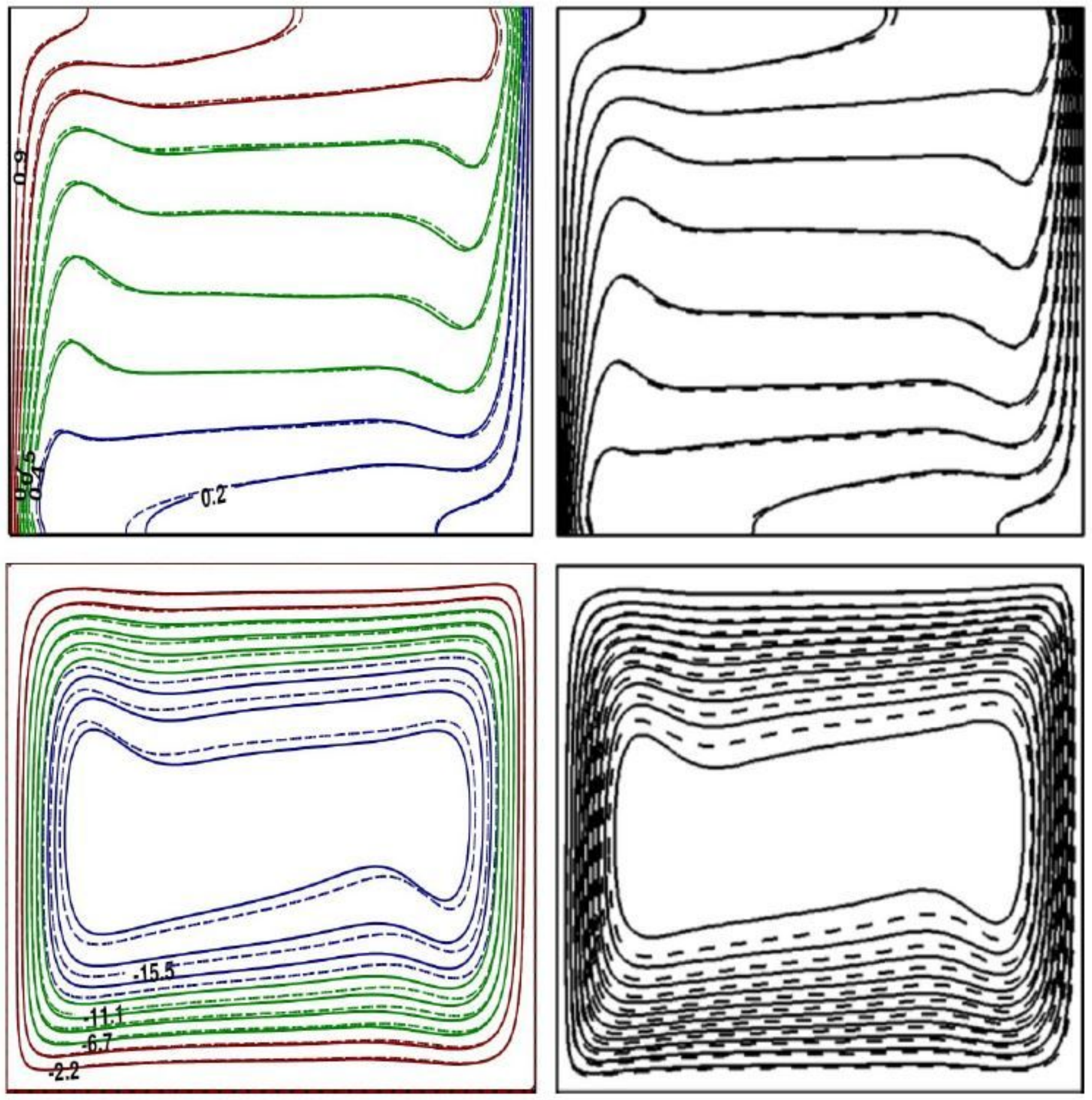

Figure 2

Comparision of present simulations (left) with the results of Ho et al.26 (right). Base fluid (dotted line) and nanofluid (solid line). (color online) 

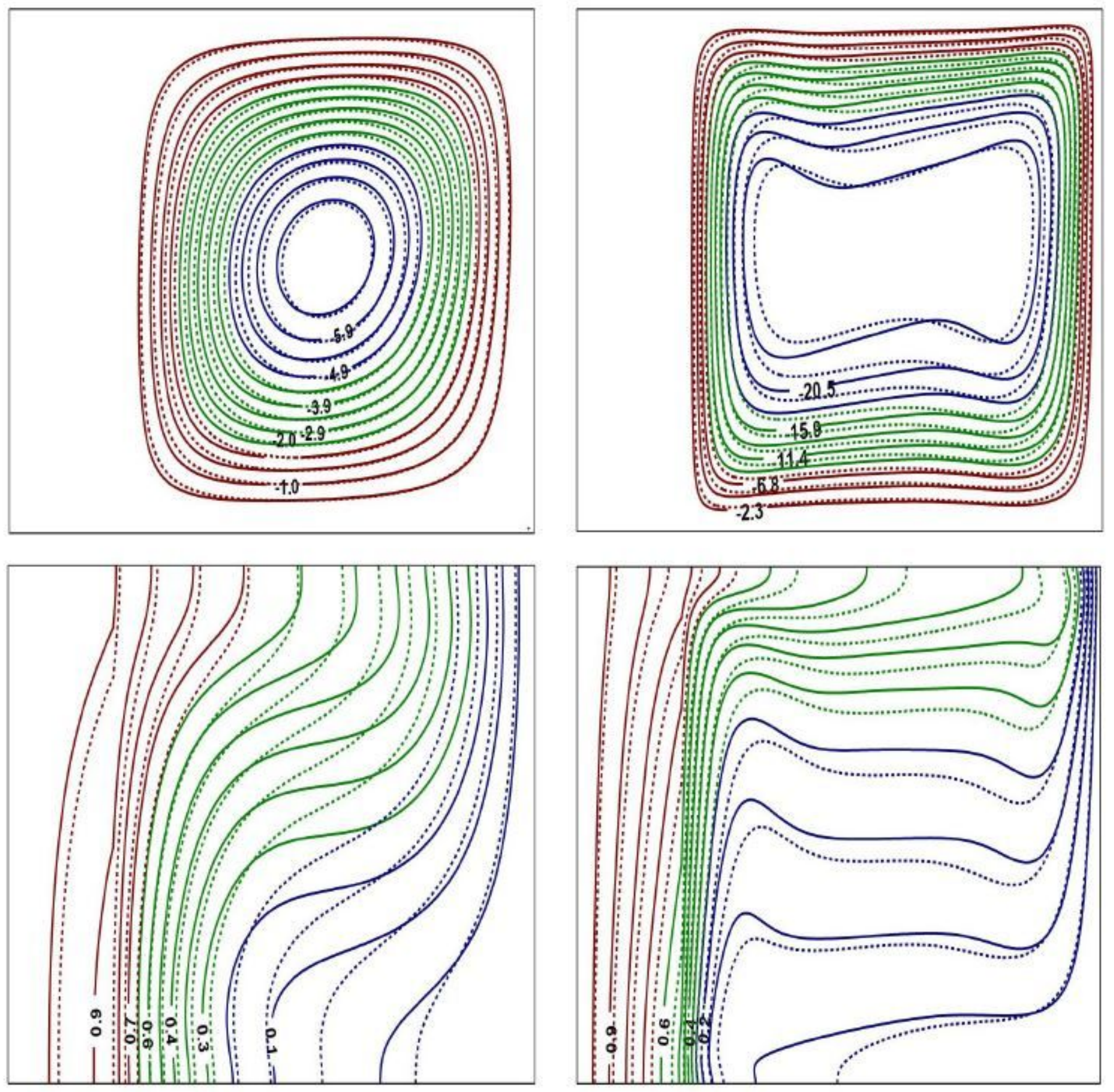

Figure 3

Impact of $\mathrm{Ra}$ on flow and thermal contours at $\mathrm{Ra}=104$ (left) and $\mathrm{Ra}=106$ (right) for base fluid (solid curve) and Al2O3-nanofluid (dotted curve) with $\varphi=0.1, \mathrm{Kr}=5$ and $\varepsilon=0.2$. (color online) 

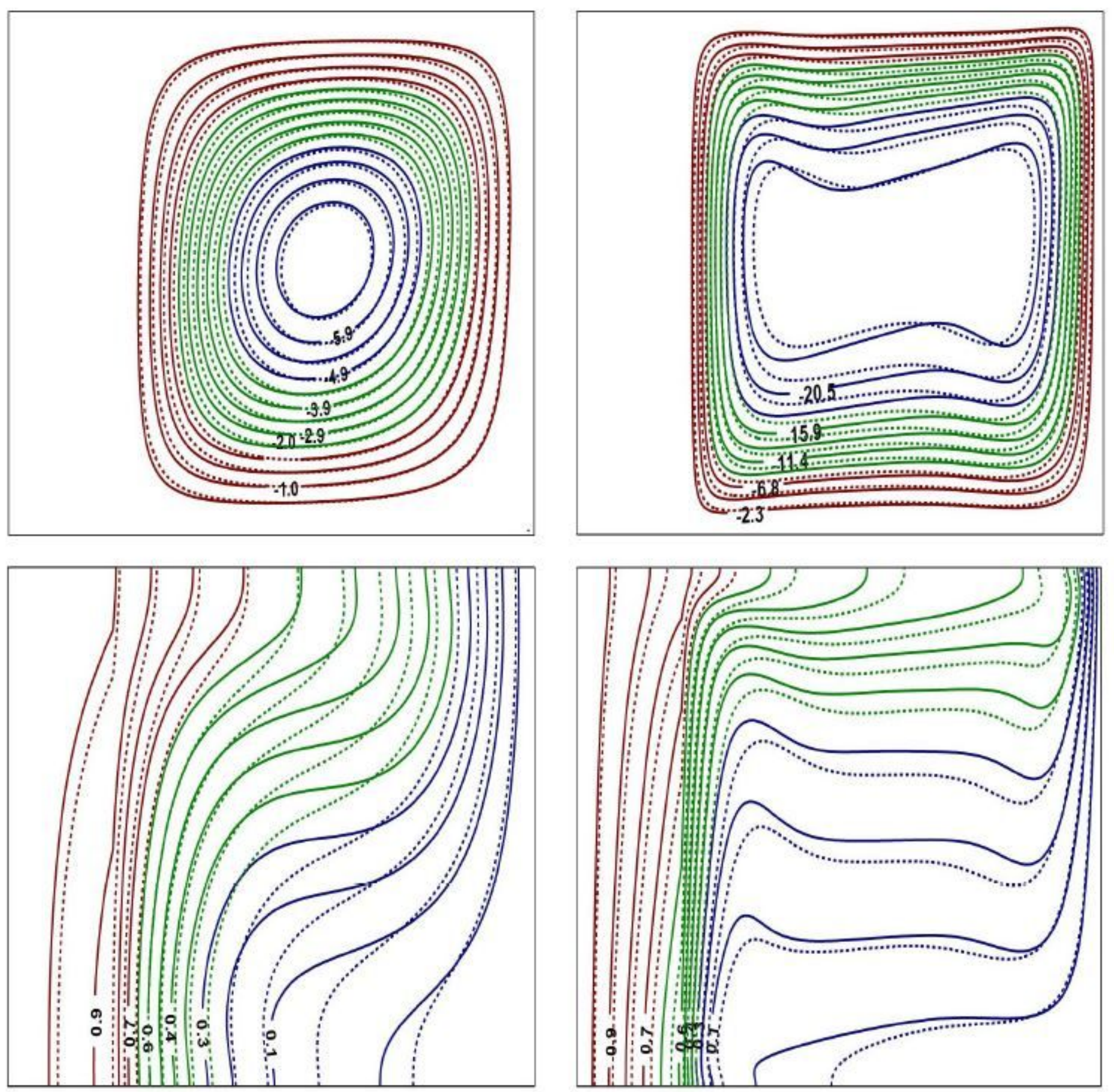

\section{Figure 4}

Impact of Ra on flow and thermal contours at $\mathrm{Ra}=104$ (left) and $\mathrm{Ra}=106$ (right) for base fluid (solid curve) and TiO2-nanofluid (dotted curve) with $\varphi=0.1, \mathrm{Kr}=5$ and $\varepsilon=0.2$. (color online) 

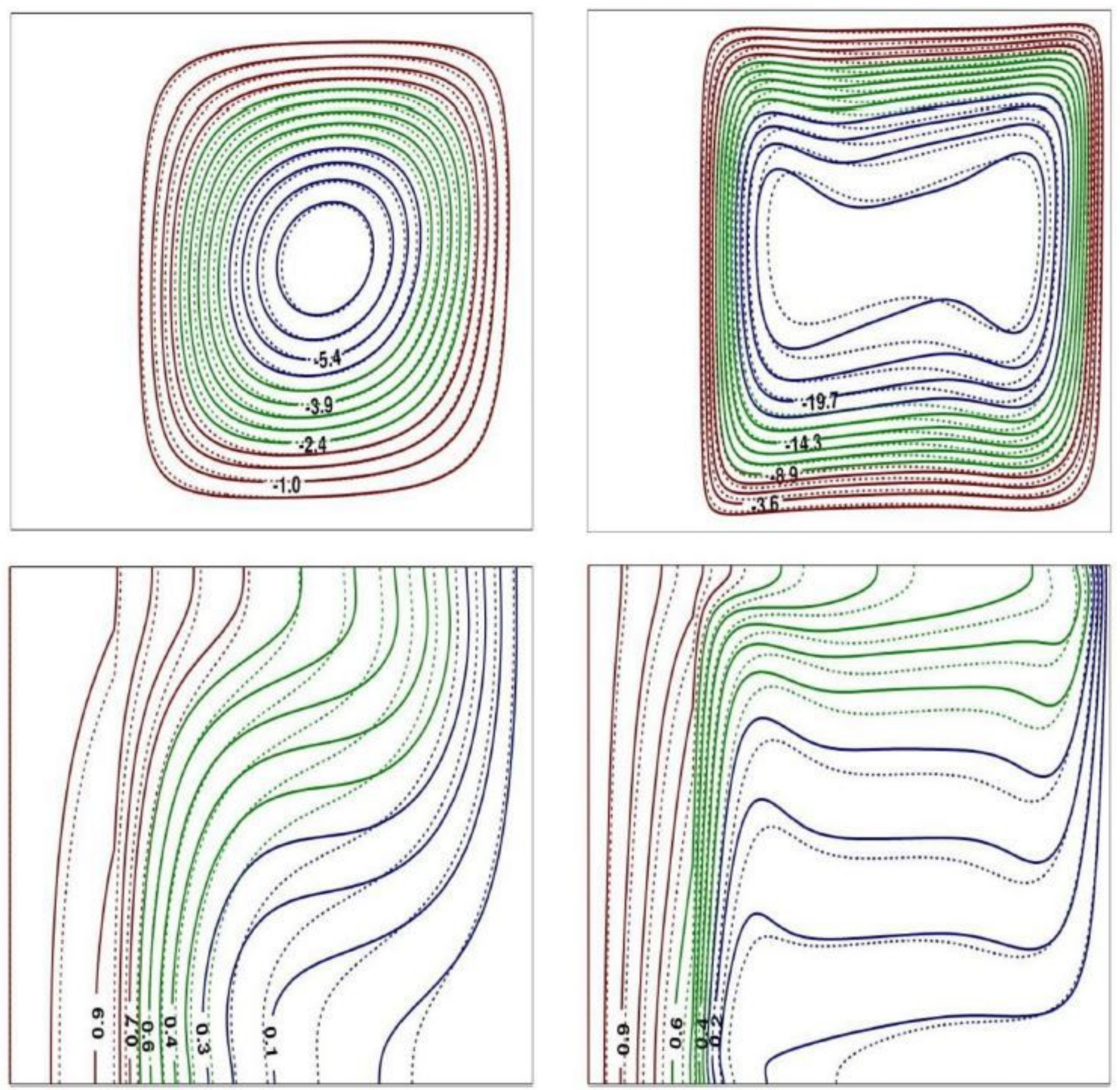

\section{Figure 5}

Impact of $\mathrm{Ra}$ on flow and thermal contours at $\mathrm{Ra}=104$ (left) and $\mathrm{Ra}=106$ (right) for base fluid (solid curve) and Cu-nanofluid (dotted curve) with $\varphi=0.1, \mathrm{Kr}=5$ and $\varepsilon=0.2$. (color online) 

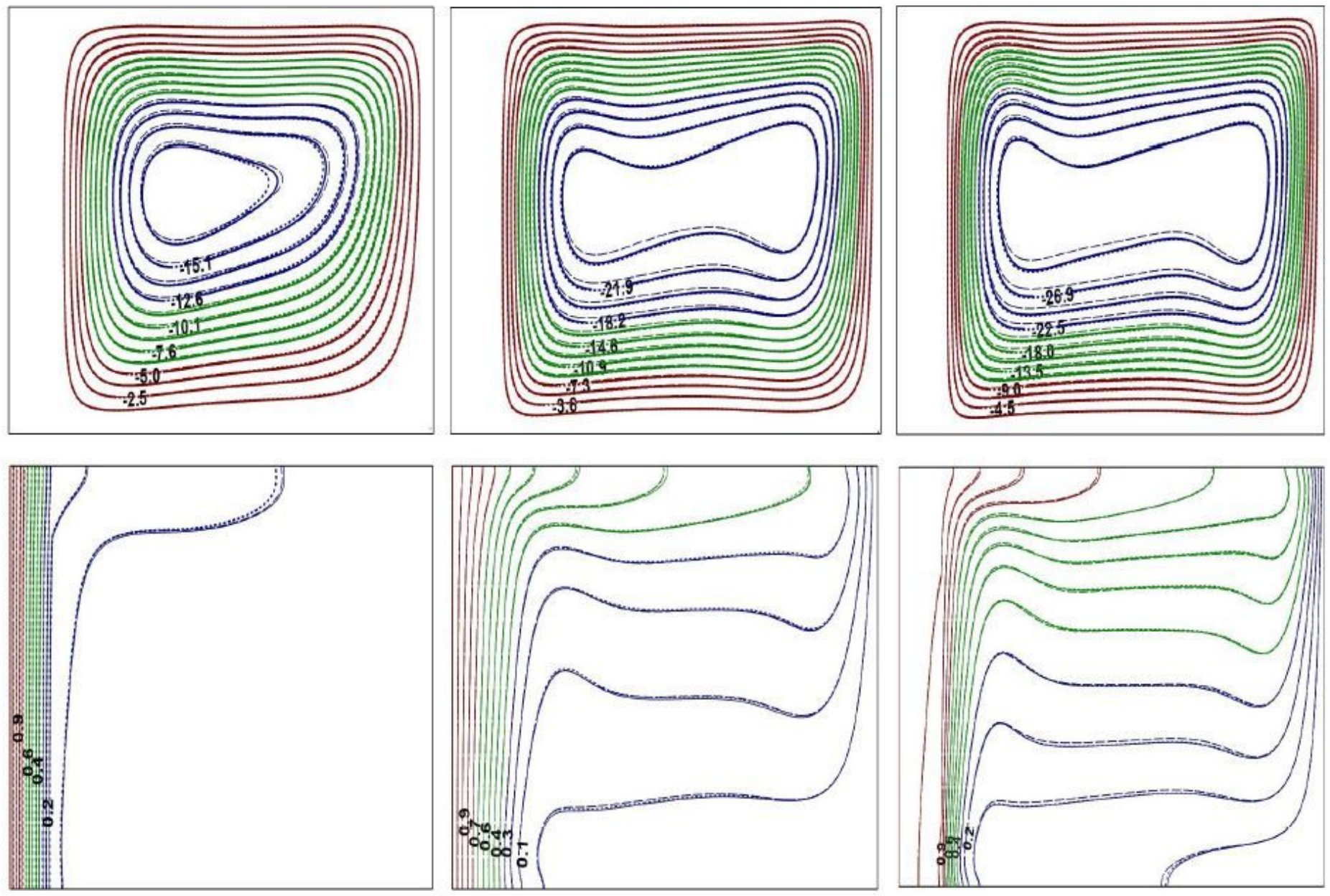

Figure 6

Impact of $\mathrm{Kr}$ on flow and thermal contours at $\mathrm{Ra}=106, \varphi=0.1$ and $\varepsilon=0.1 . \mathrm{Kr}=0.1$ (left), $\mathrm{Kr}=1.0$ (center) and $\mathrm{Kr}=10$ (right). (color online) 

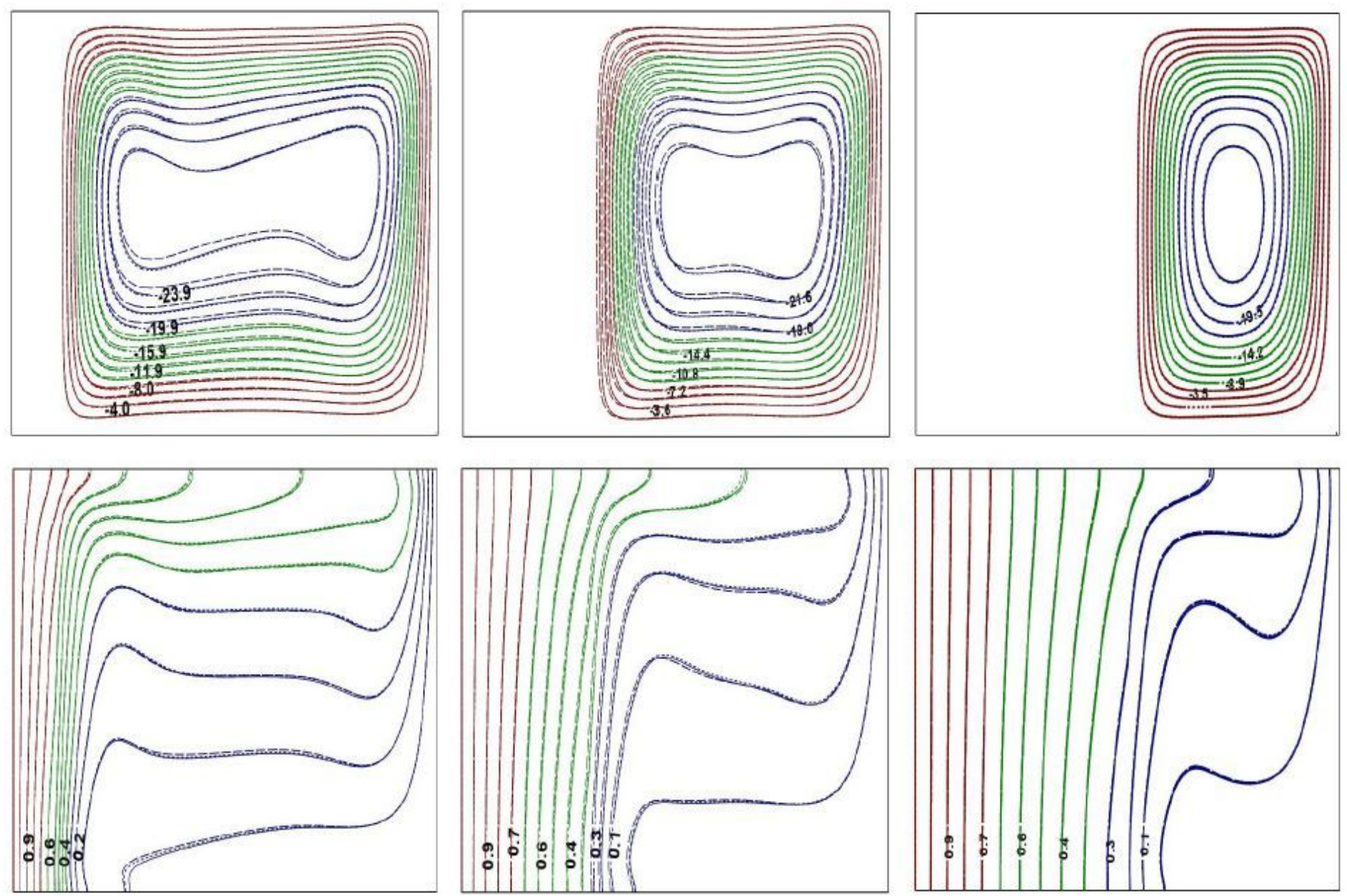

Figure 7

Impact of $\varepsilon$ on flow and thermal contours at $\mathrm{Ra}=106, \varphi=0.1$ and $\mathrm{Kr}=2 . \varepsilon=0.1$ (left), $\varepsilon=0.3$ (center) and $\varepsilon=0.5$ (right). (color online) 

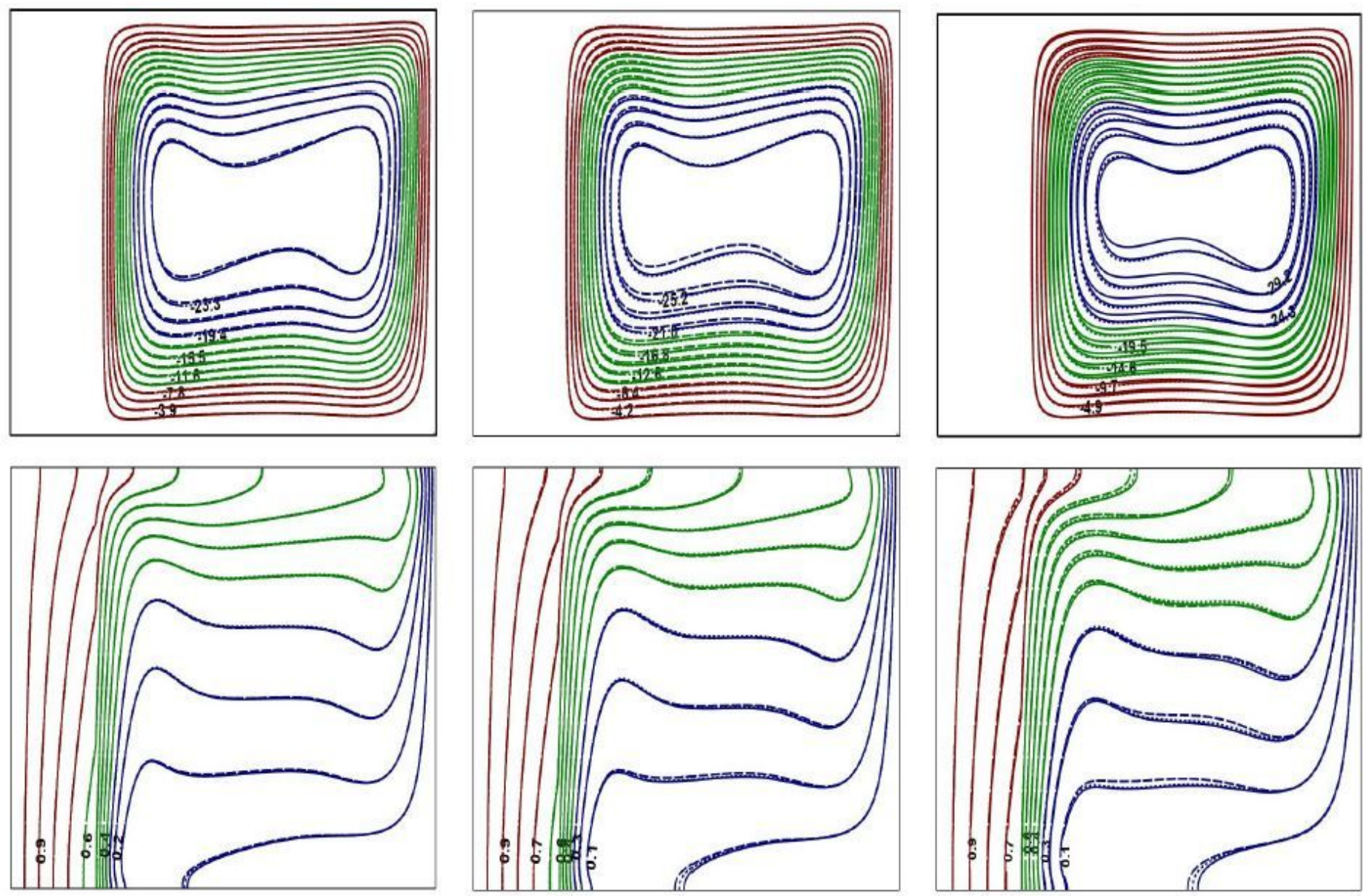

Figure 8

Impact of $\varphi$ on flow and thermal contours at $\mathrm{Ra}=106, \varepsilon=0.2$ and $\mathrm{Kr}=5 . \varphi=0.05$ (left), $\varphi=0.1$ (center) and $\varphi=0.2$ (right). (color online) 


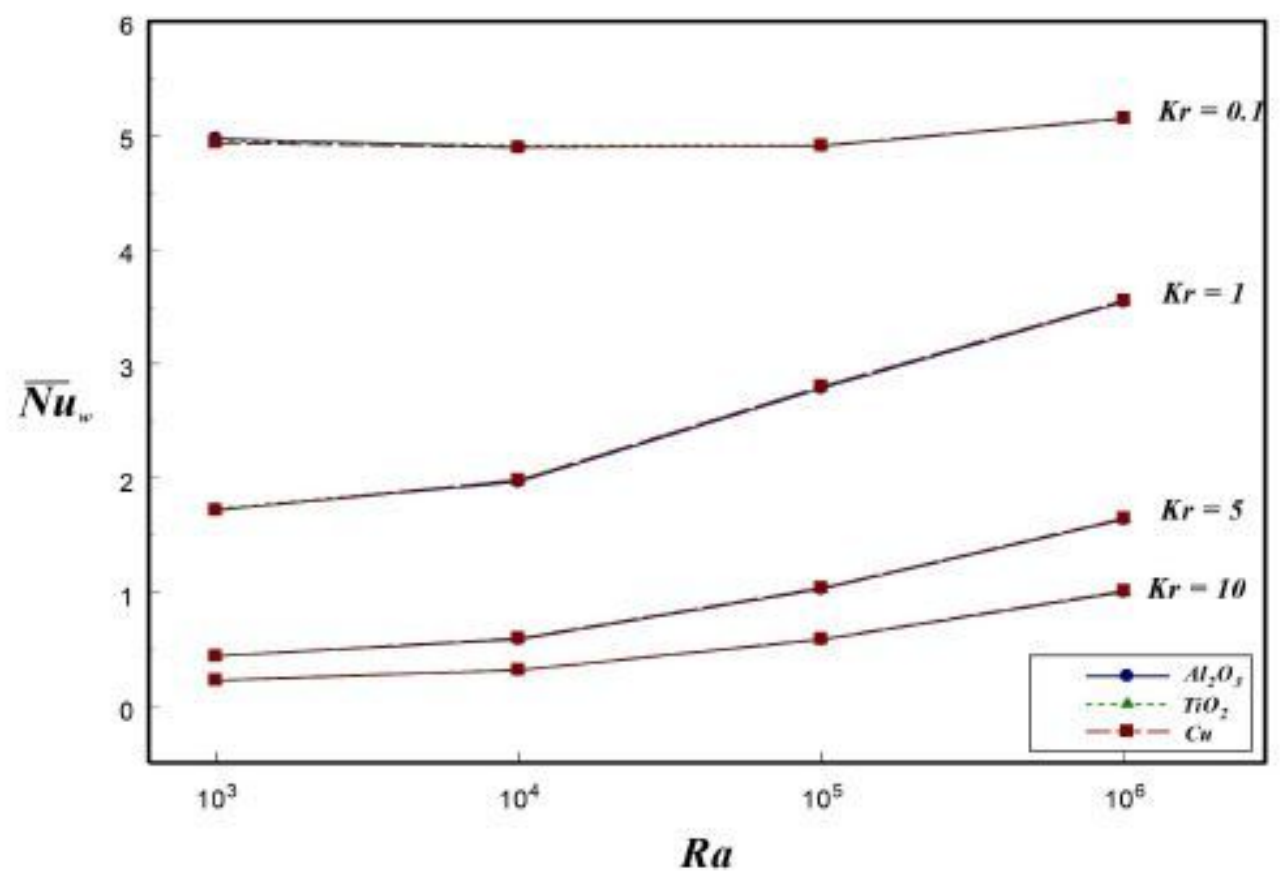

(a)

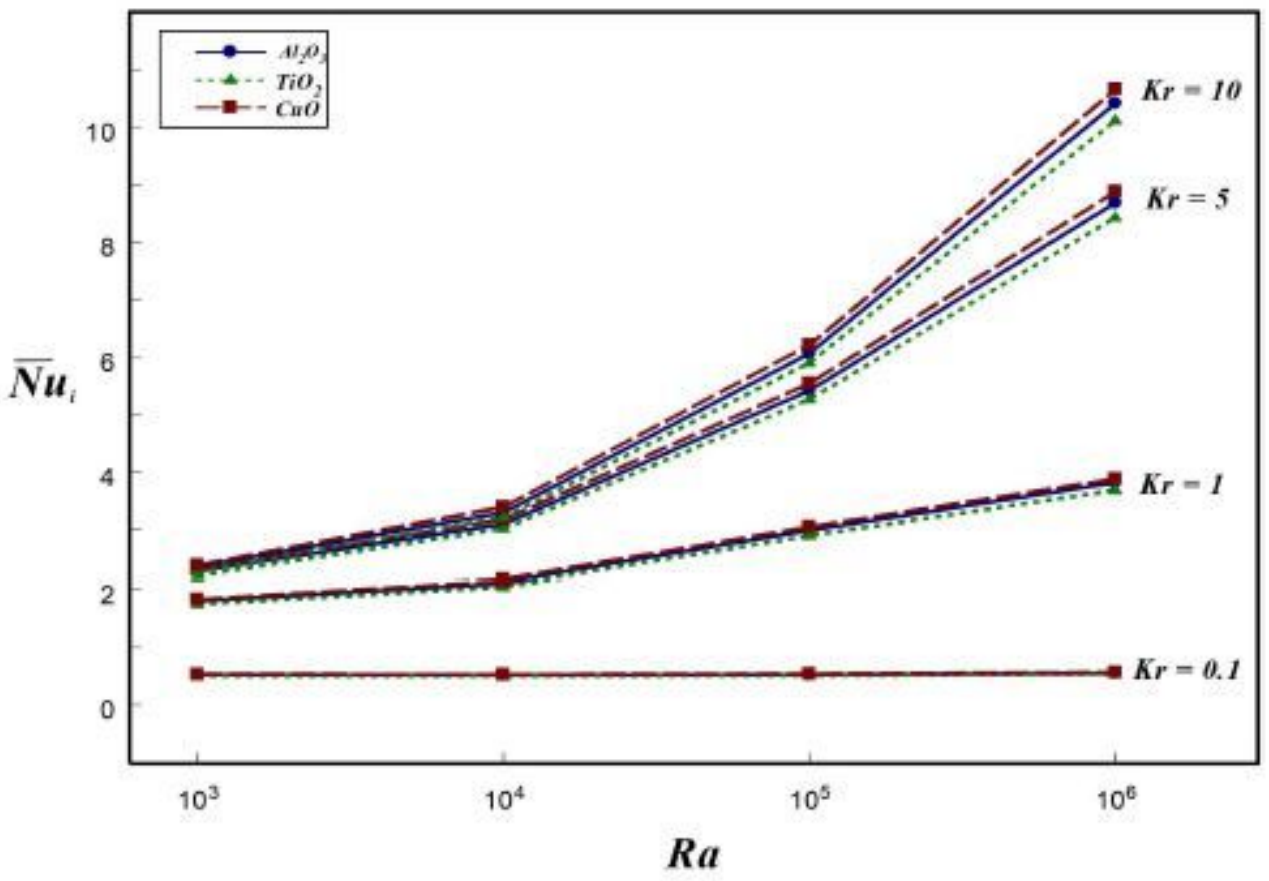

(b)

Figure 9

Effect of Ra and $\mathrm{Kr}$ on Nu at the (a) wall and (b) interface for $\varepsilon=0.2$ and $\varphi=0.1$. (color online) 


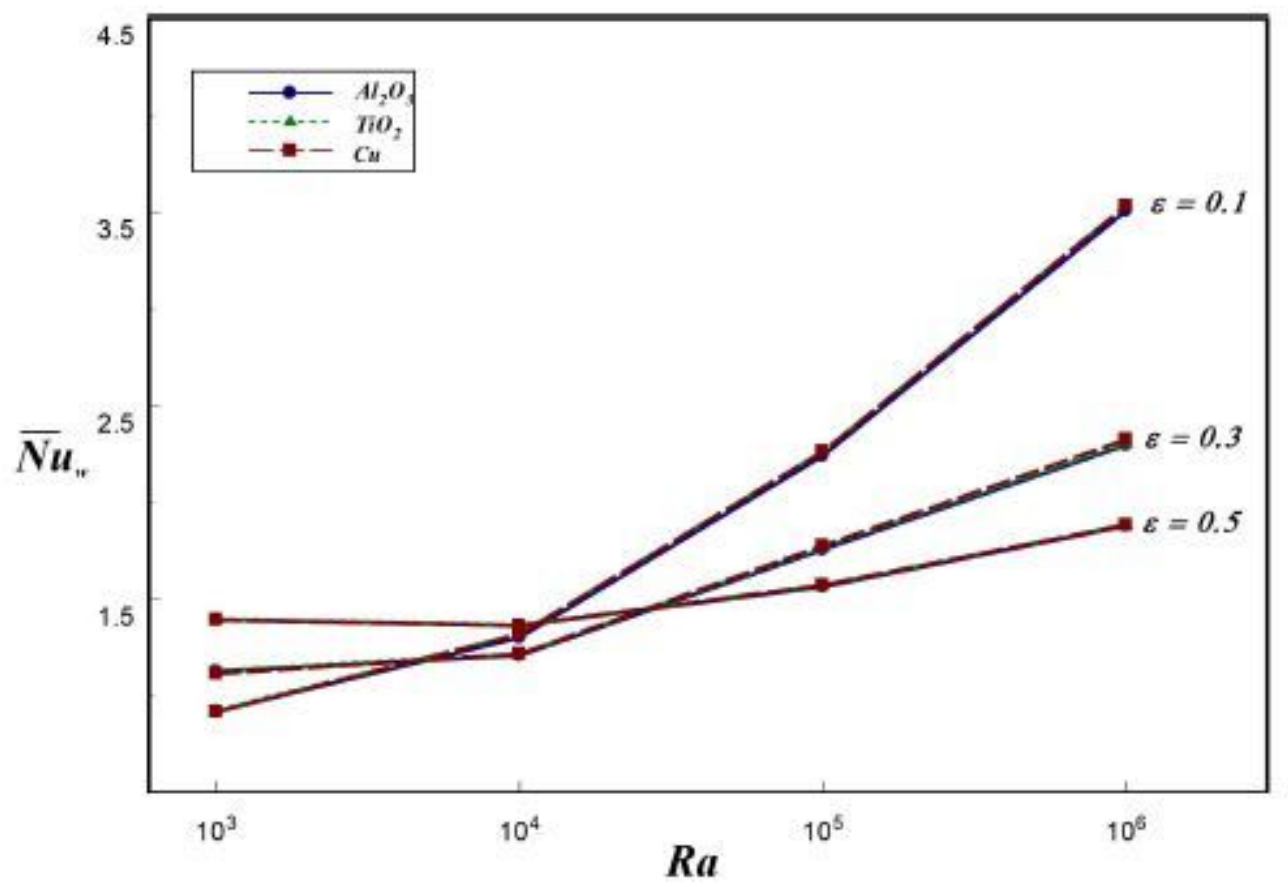

(a)

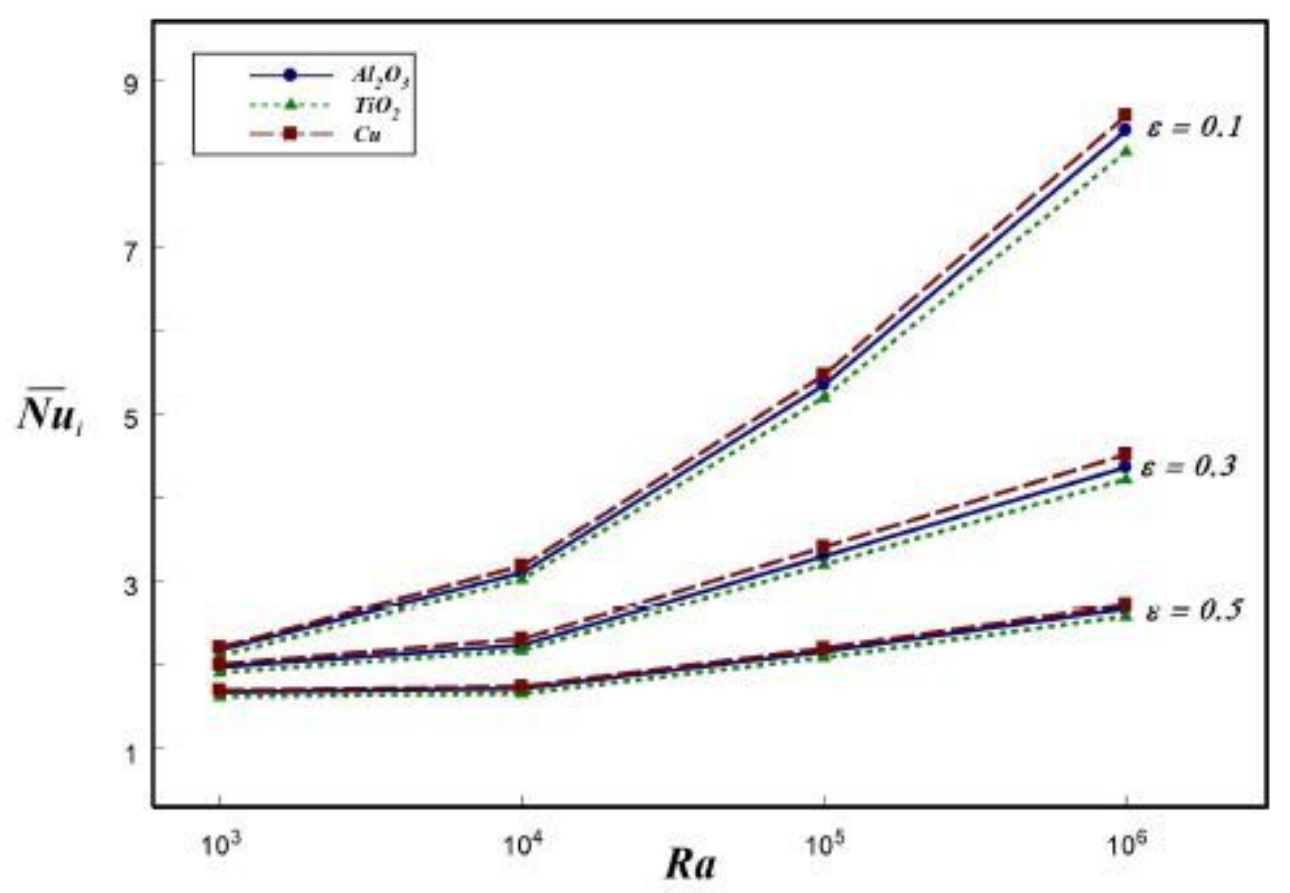

(b)

Figure 10

Effect of Ra and $\varepsilon$ on $\mathrm{Nu}$ at the (a) wall and (b) interface for $\mathrm{Kr}=2$ and $\varphi=0.1$. (color online) 


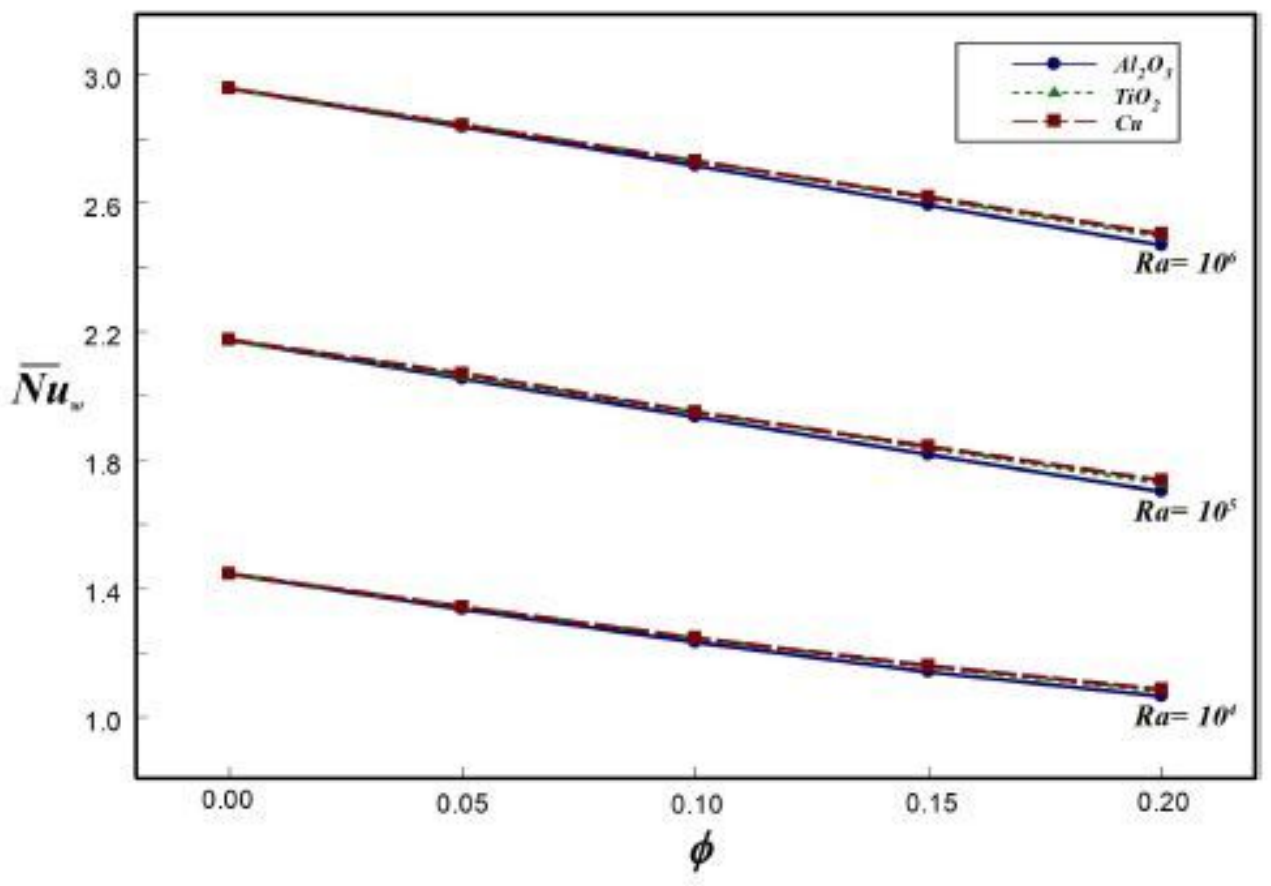

(a)

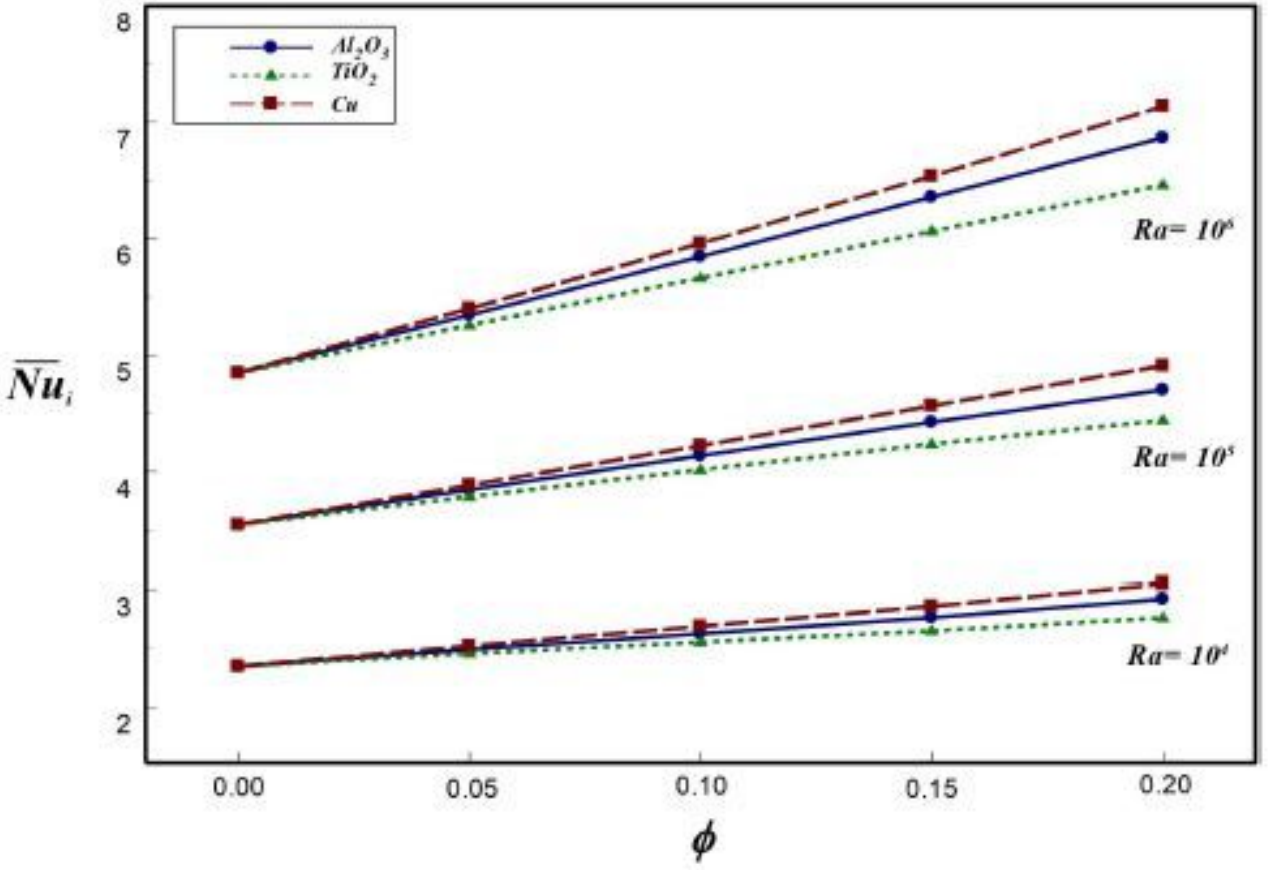

(b)

Figure 11

Effect of $\mathrm{Ra}$ and $\varphi$ on $\mathrm{Nu}$ at the (a) wall and (b) interface for $\mathrm{Kr}=2$ and $\varepsilon=0.2$. (color online) 


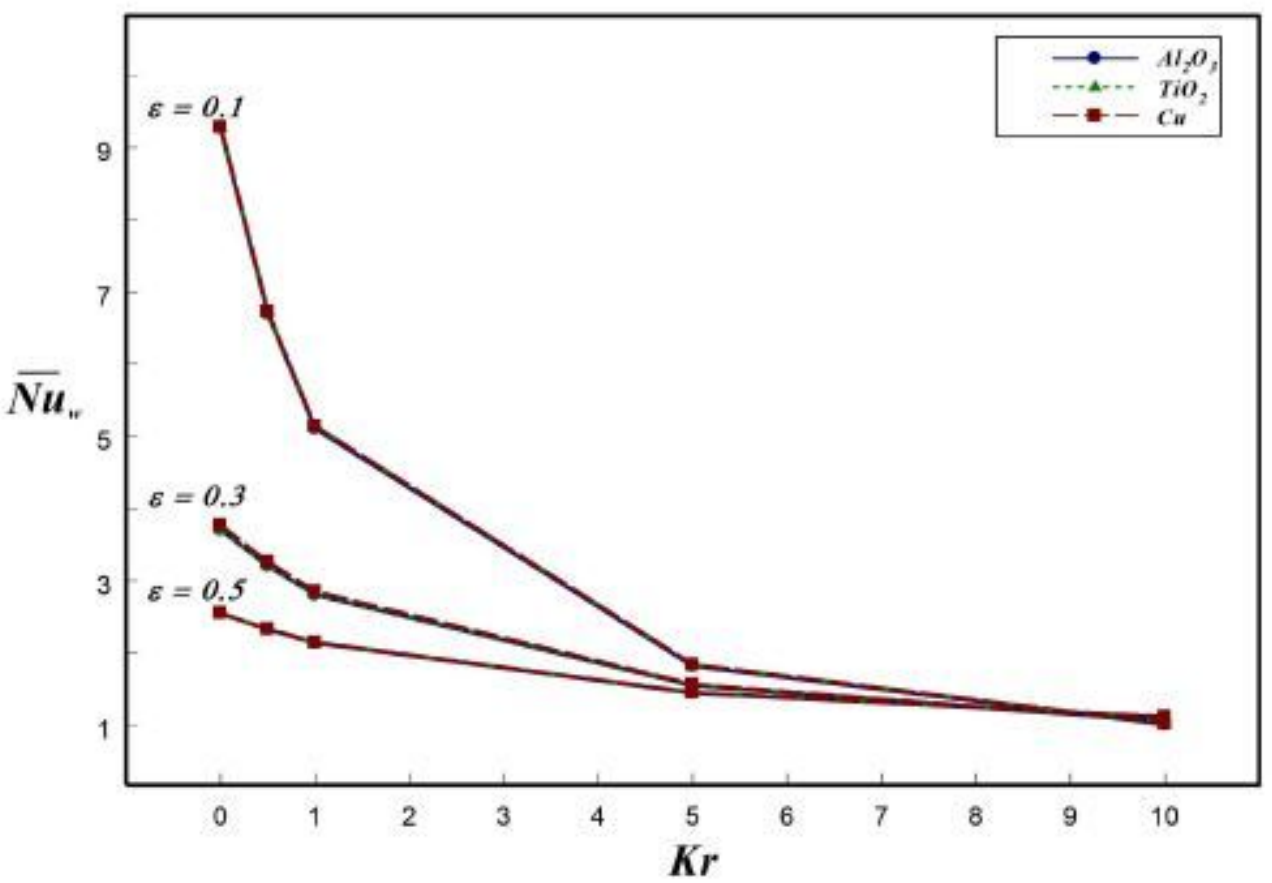

(a)

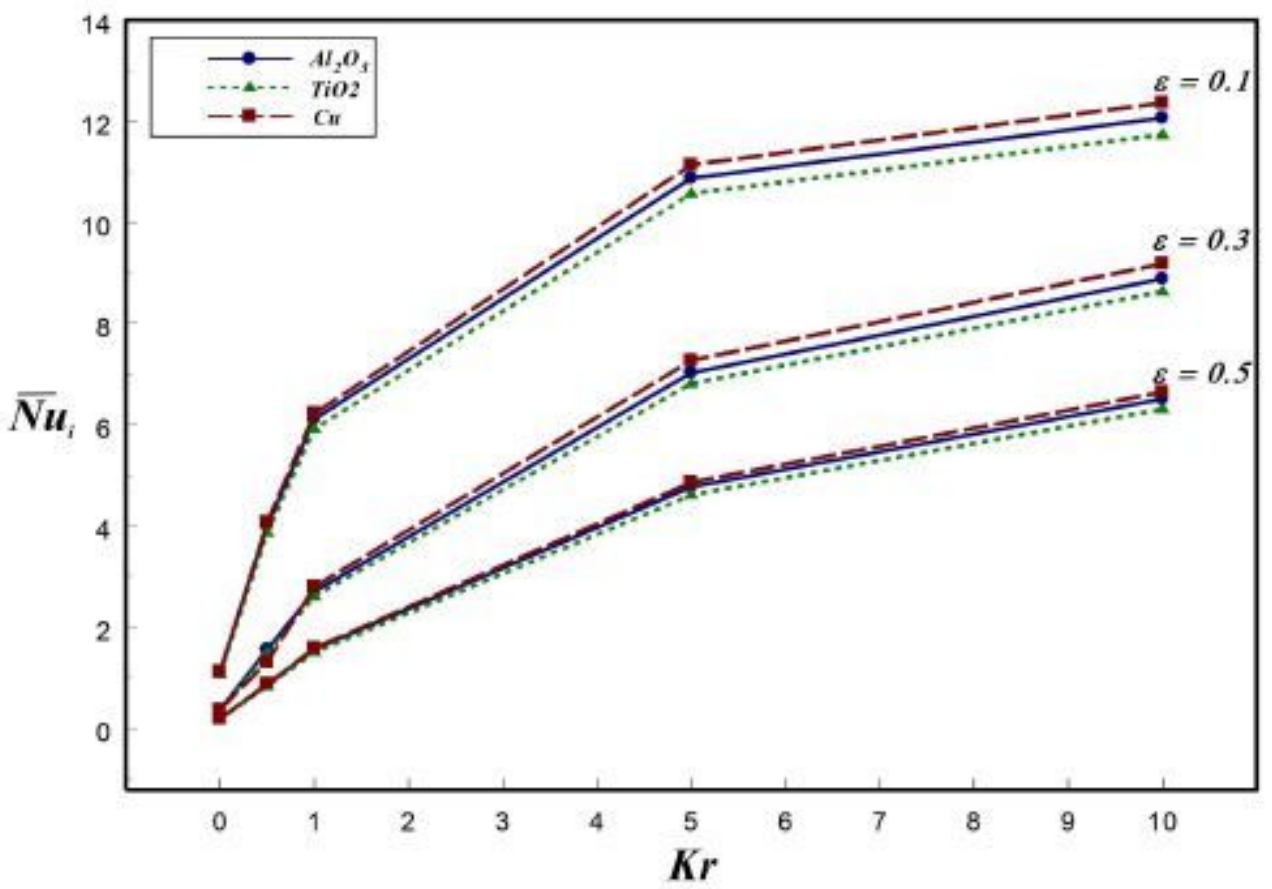

(b)

Figure 12

Effect of $\mathrm{Kr}$ and $\varepsilon$ on $\mathrm{Nu}$ at the (a) wall and (b) interface for $\mathrm{Ra}=106$ and $\varphi=0.1$. (color online) 


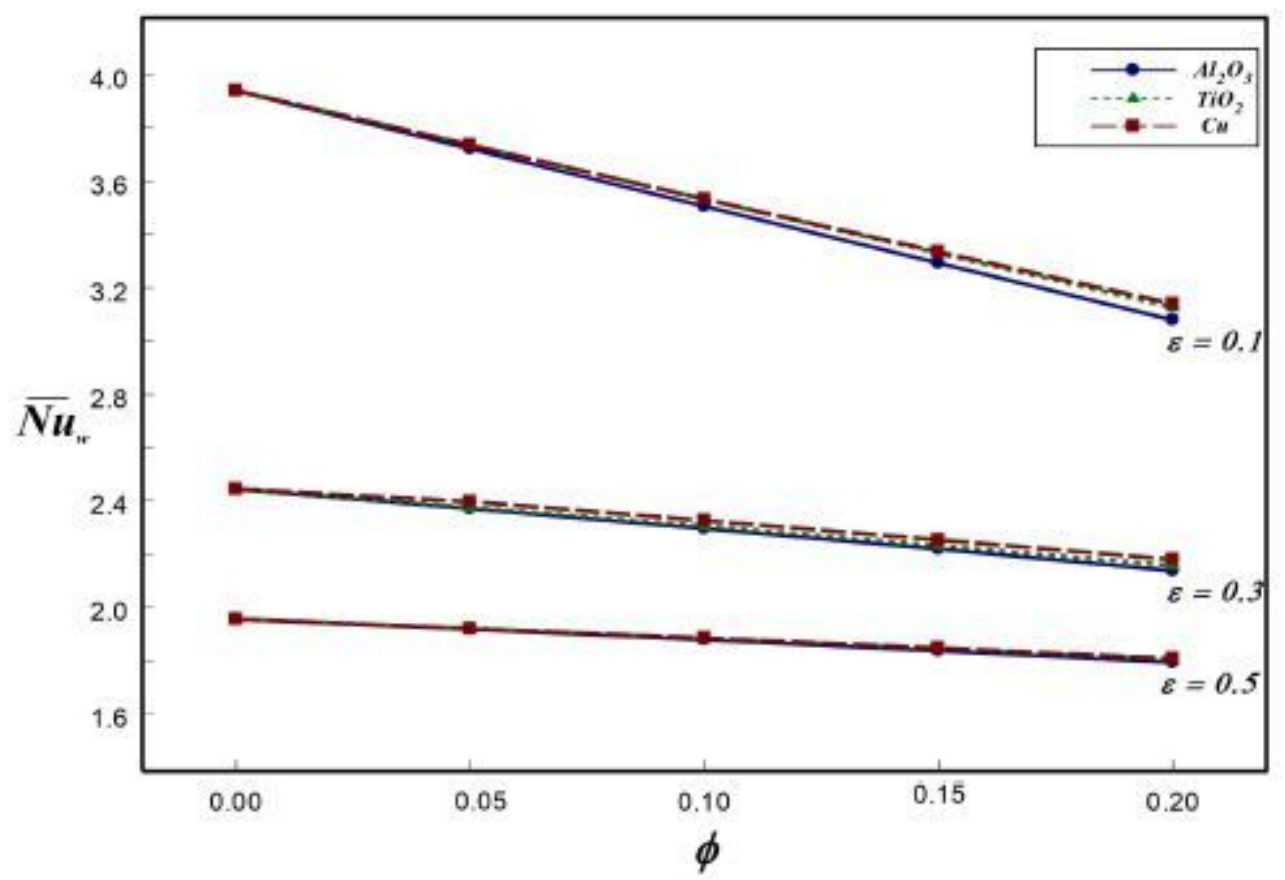

(a)

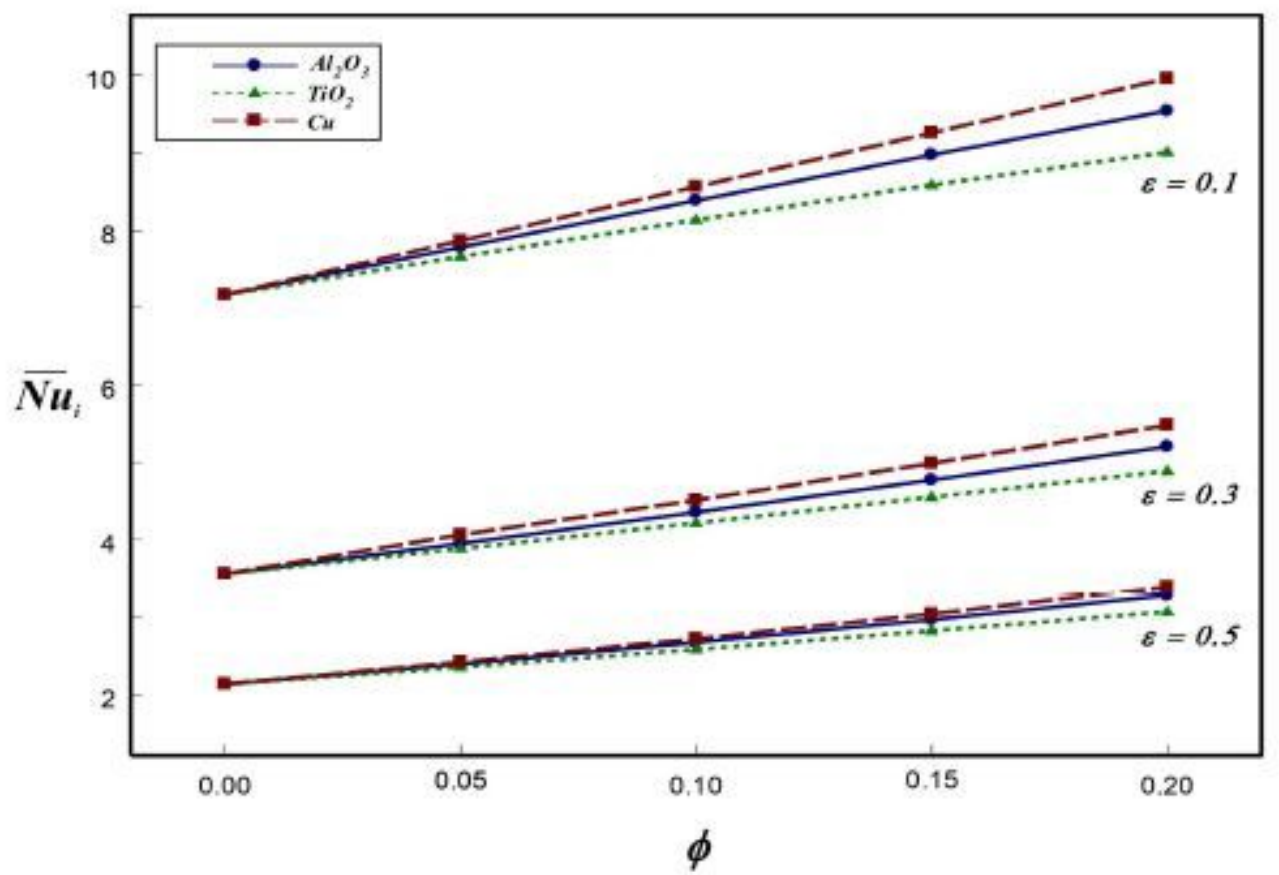

(b)

Figure 13

Effect of $\varphi$ and $\varepsilon$ on $\mathrm{Nu}$ at the (a) wall and (b) interface for $\mathrm{Ra}=106$ and $\mathrm{Kr}=2$. (color online) 


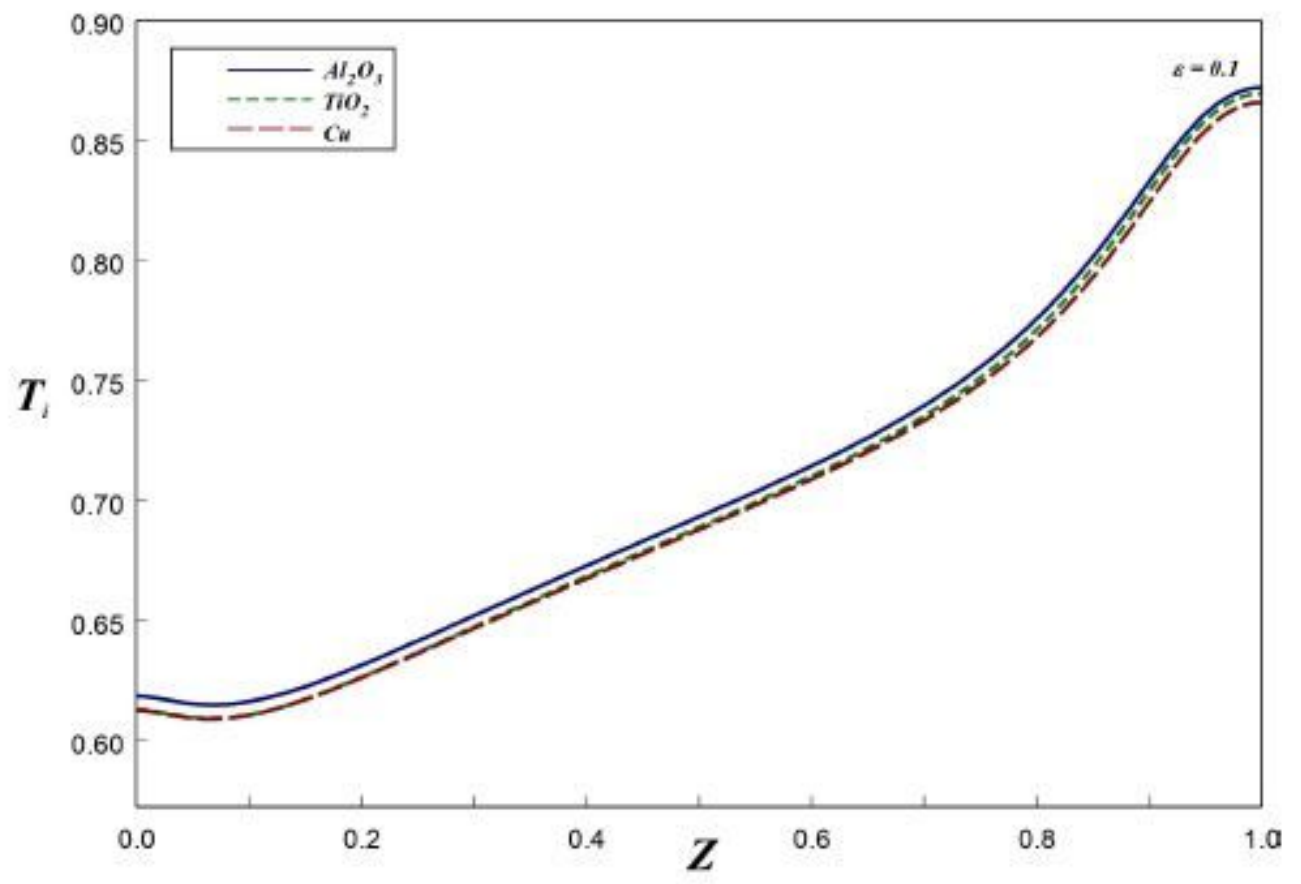

(a)

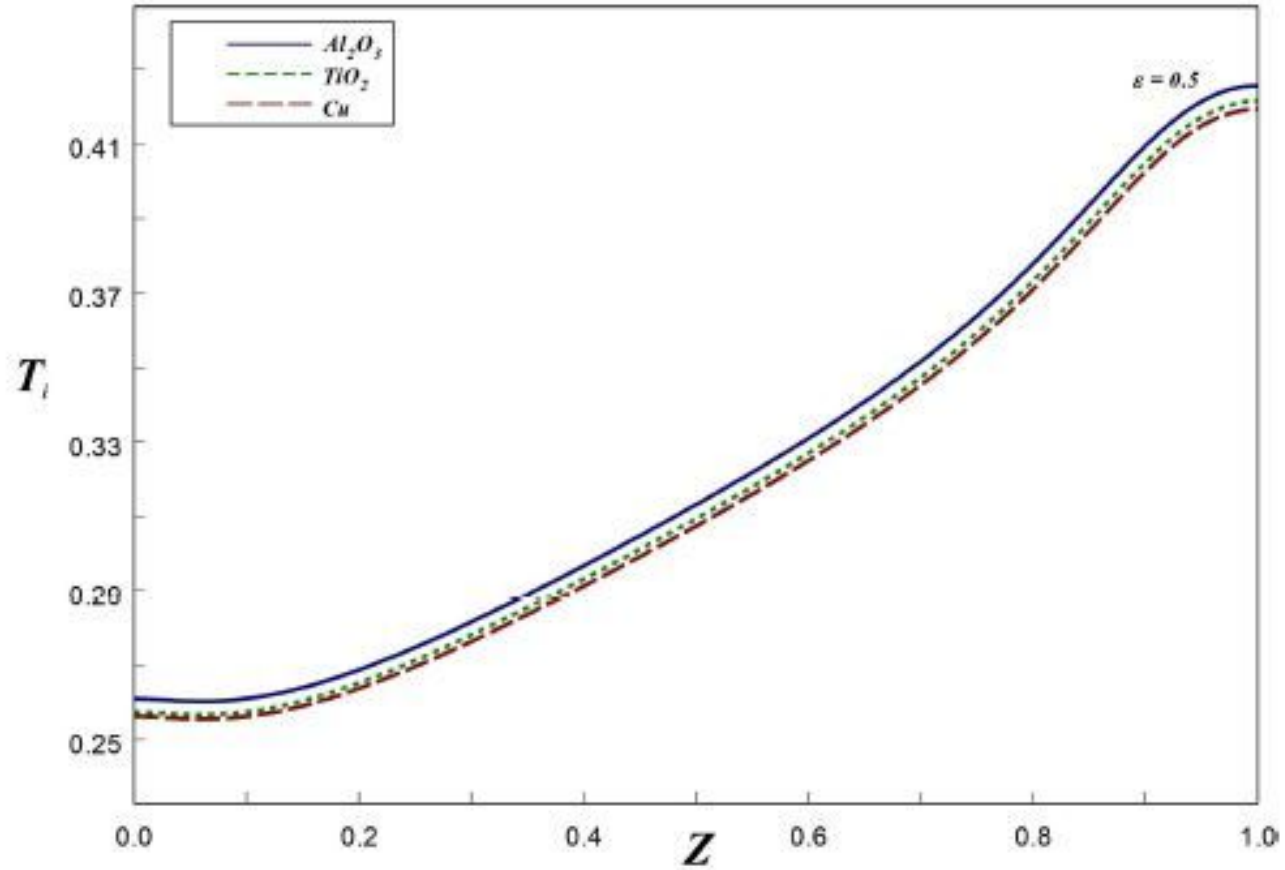

(b)

\section{Figure 14}

Axial variation of interface temperature with different wall thickness (a) $\varepsilon=0.1$ and (b) $\varepsilon=0.5$. (color online) 


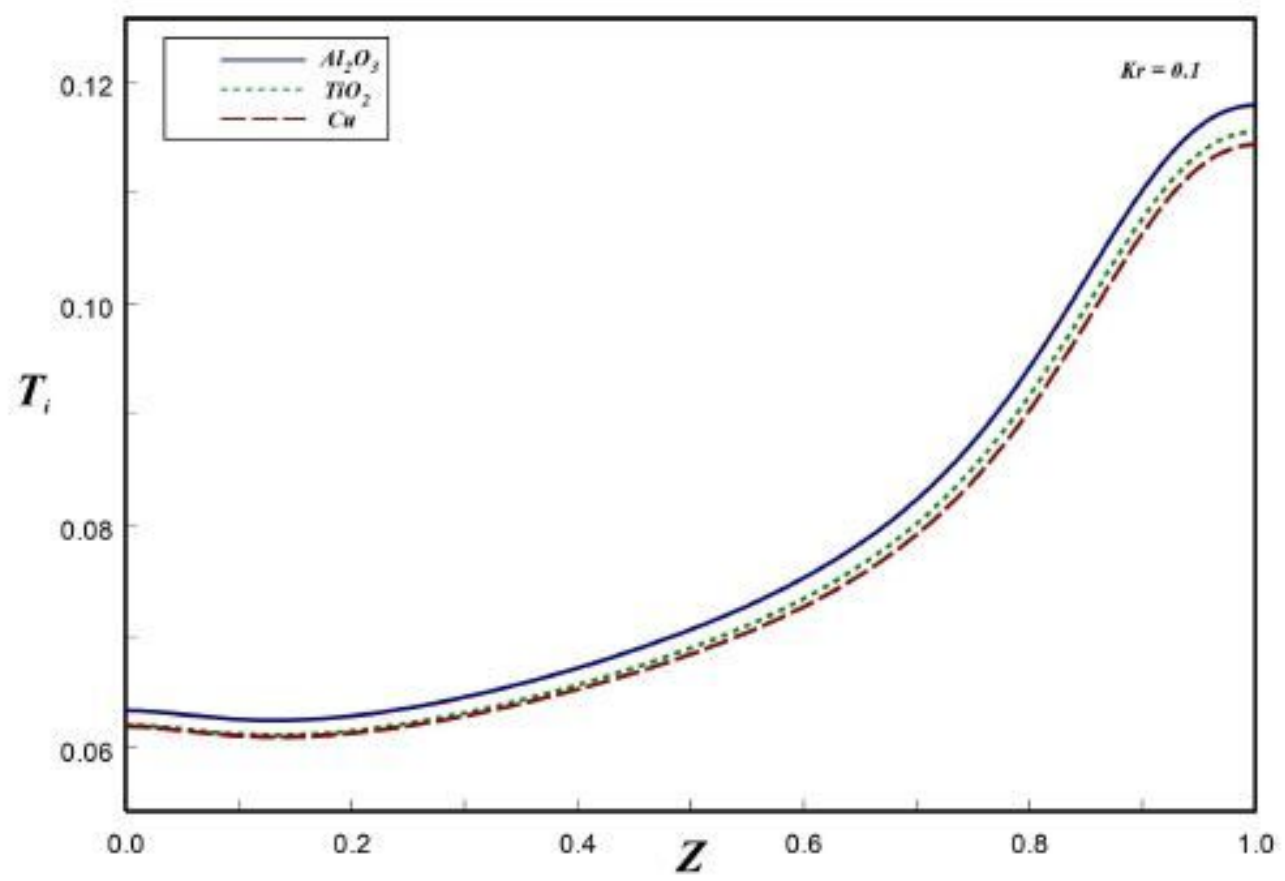

(a)

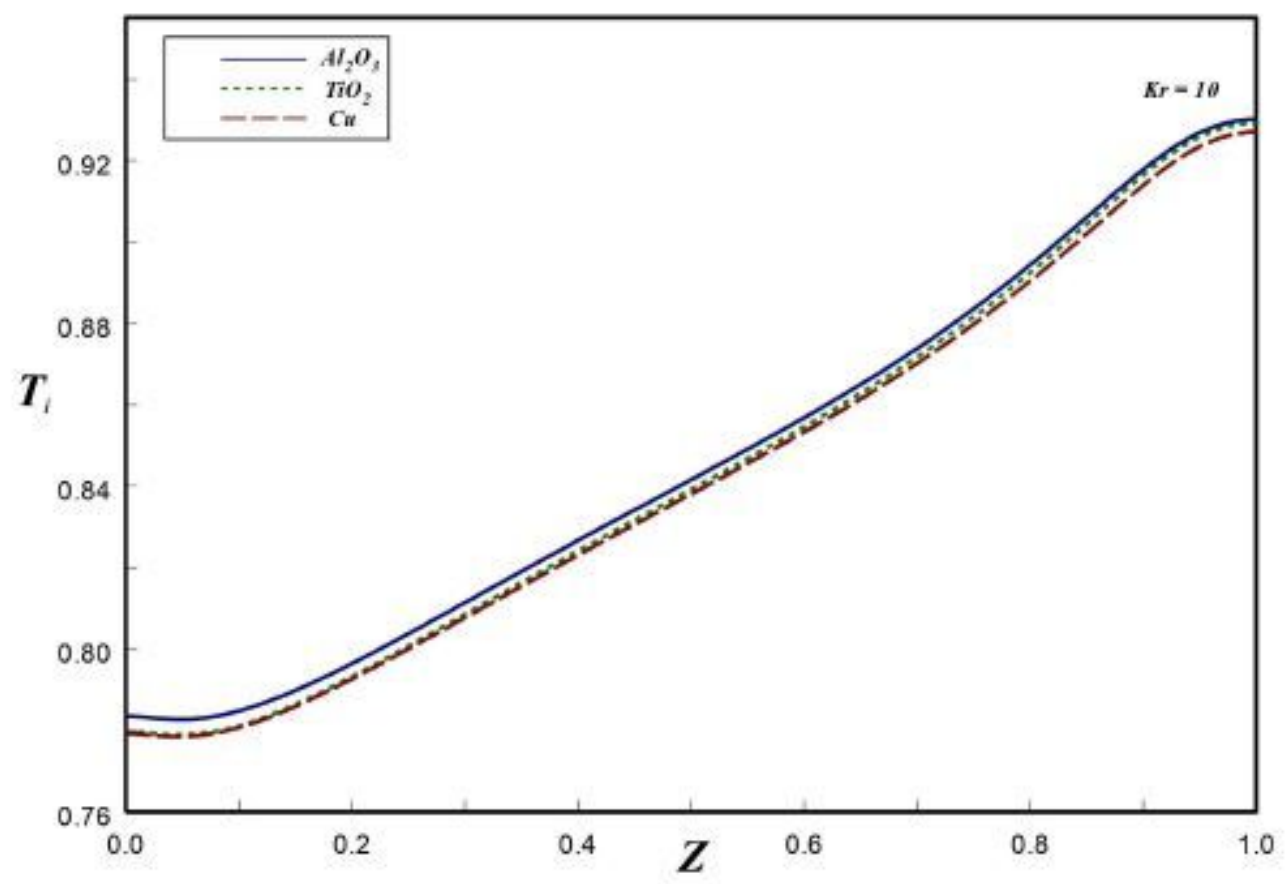

(b)

\section{Figure 15}

Axial variation of interface temperature with different thermal conductivity ratio (a) $\mathrm{Kr}=0.1$ and (b) $\mathrm{Kr}=$ 10. (color online) 


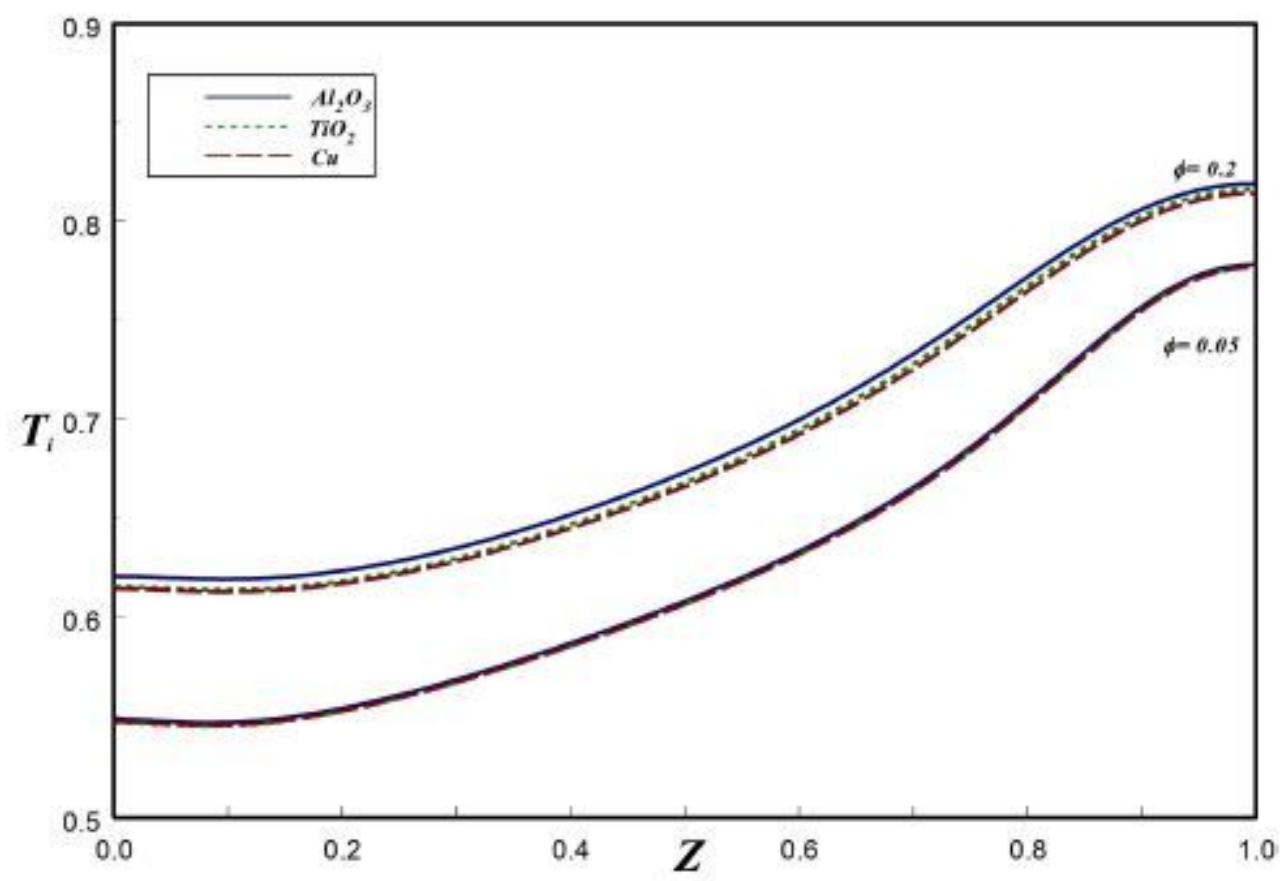

(a)

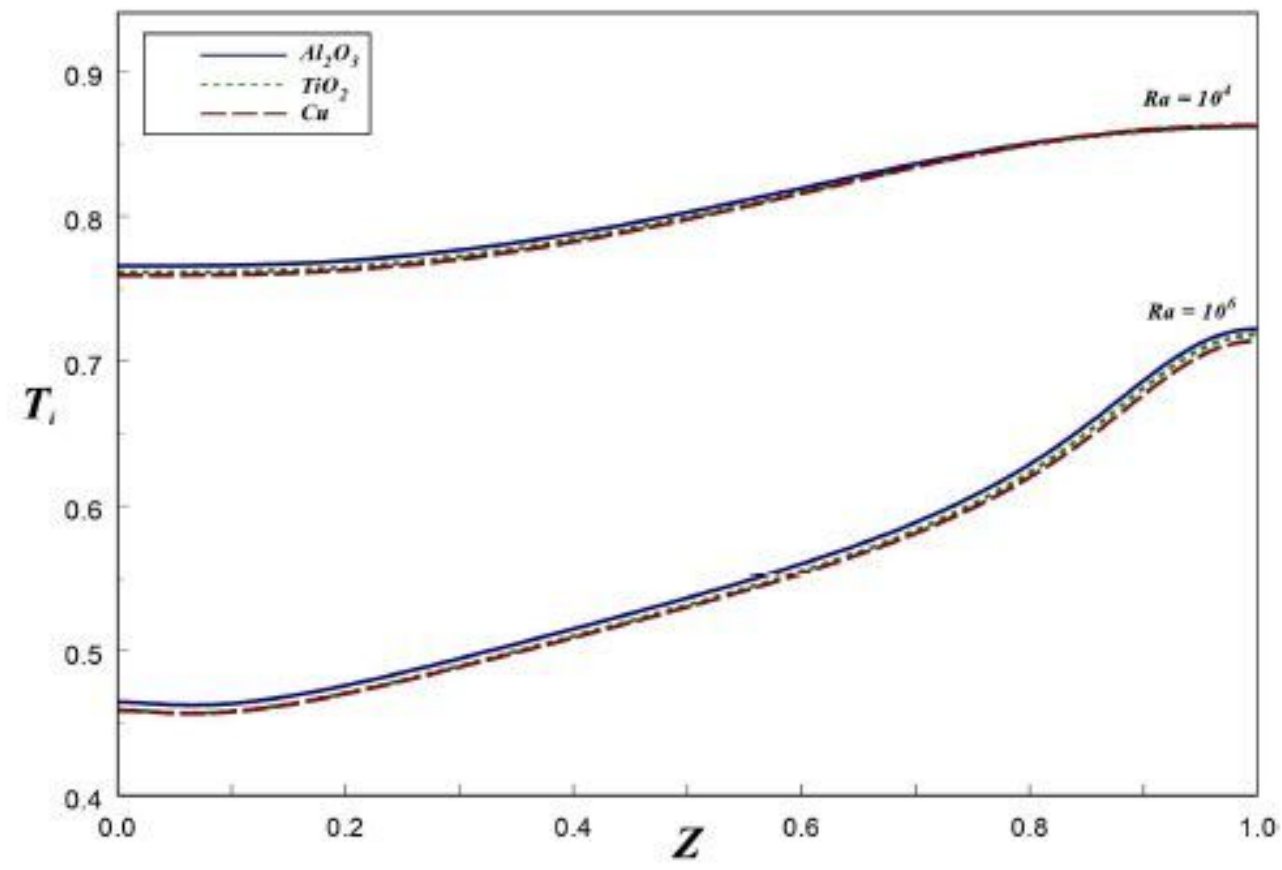

(b)

\section{Figure 16}

Axial variation of interface temperature with different (a) nanoparticle volume fraction $(\varphi)$ and (b) Rayleigh number $(\mathrm{Ra})$. (color online) 